TRANSACTIONS OF THE

AMERICAN MATHEMATICAL SOCIETY

Volume 351, Number 1, January 1999, Pages 1-40

S 0002-9947(99)02180-7

\title{
SIMPLICITY AND THE STABLE RANK OF SOME FREE PRODUCT $\mathrm{C}^{*}$-ALGEBRAS
}

\author{
KENNETH J. DYKEMA
}

\begin{abstract}
A necessary and sufficient condition for the simplicity of the $\mathrm{C}^{*}$ algebra reduced free product of finite dimensional abelian algebras is found, and it is proved that the stable rank of every such free product is 1 . Related results about other reduced free products of $\mathrm{C}^{*}$-algebras are proved.
\end{abstract}

\section{INTRODUCTION}

The reduced free product of $\mathrm{C}^{*}$-algebras with respect to given states was introduced independently by Voiculescu [19] and Avitzour [2]. It is the appropriate construction associated to Voiculescu's free probability theory (see [19], [21]). The motivating example concerns reduced group $\mathrm{C}^{*}$-algebras. For a discrete group $G$, its reduced group $\mathrm{C}^{*}$-algebra is generated by the left regular representation of $G$ on $l^{2}(G)$ and is denoted $C_{r}^{*}(G)$. Its canonical tracial state (which is the vector state associated to the characteristic function of the identity element of $G$ ) is written $\tau_{G}$. Then for discrete groups $G_{1}$ and $G_{2}$, the reduced free product construction yields

$$
\left(C_{r}^{*}\left(G_{1}\right), \tau_{G_{1}}\right) *\left(C_{r}^{*}\left(G_{2}\right), \tau_{G_{2}}\right)=\left(C_{r}^{*}(G), \tau_{G}\right),
$$

where $G=G_{1} * G_{2}$ is the free product of groups.

Voiculescu's definition of freeness is an abstraction of some essential facets of the relationship between the copies of $C_{r}^{*}\left(G_{1}\right)$ and $C_{r}^{*}\left(G_{2}\right)$ embedded in $C_{r}^{*}(G)$, with respect to the trace $\tau_{G}$. The reduced free product of $\mathrm{C}^{*}$-algebras can be described with respect to freeness as follows. Let $A_{1}$ and $A_{2}$ be unital $\mathrm{C}^{*}$-algebras with states $\phi_{1}$ and $\phi_{2}$, respectively whose associated GNS representations are faithful. Then the reduced free product of $\left(A_{1}, \phi_{1}\right)$ and $\left(A_{2}, \phi_{2}\right)$ is the (unique) unital $\mathrm{C}^{*}$-algebra $\mathfrak{A}$ and state $\phi$ with unital embeddings $A_{\iota} \hookrightarrow \mathfrak{A}$ such that

(1) the GNS representation associated to $\phi$ is faithful on $\mathfrak{A}$;

(2) $\left.\phi\right|_{A_{\iota}}=\phi_{\iota}$;

(3) $A_{1}$ and $A_{2}$ are free with respect to $\phi$;

(4) $\mathfrak{A}$ is generated by $A_{1} \cup A_{2}$.

We denote this by

$$
(\mathfrak{A}, \phi)=\left(A_{1}, \phi_{1}\right) *\left(A_{2}, \phi_{2}\right) .
$$

It is further known [19] (or see [21, 2.5.3]) that $\phi$ is a trace if $\phi_{1}$ and $\phi_{2}$ are traces. Moreover, by [7], $\phi$ is also faithful on $\mathfrak{A}$ if $\phi_{1}$ and $\phi_{2}$ are faithful.

The reduced free product thus provides a multitude of constructions of $\mathrm{C}^{*}$ algebras, about which some results are known (see [19], [2], [11], [10], [9]). For

Received by the editors January 21, 1997.

1991 Mathematics Subject Classification. Primary 46L05, 46L35. 
example, many can be distinguished one from the other using K-theory, (see [11] and [12]). However, questions abound.

Perhaps the most basic question concerns simplicity of reduced free product $\mathrm{C}^{*}$ algebras. In [15], R.T. Powers showed that the reduced group $\mathrm{C}^{*}$-algebra of the free group on two generators, $C_{r}^{*}\left(F_{2}\right)$, is simple and has unique tracial state. Paschke and Salinas [14] then proved the same for $C_{r}^{*}(G)$ whenever $G=G_{1} * G_{2}$ is the free product of groups, where $G_{1}$ has at least two elements and $G_{2}$ has at least three. Avitzour [2] generalized further and showed that, for the reduced free product (1), $\mathfrak{A}$ is simple if there are unitaries $u, v \in A_{1}$ and $w \in A_{2}$ such that

$$
\begin{aligned}
\phi_{1}(u)=\phi_{1}(v) & =0=\phi_{1}\left(u^{*} v\right), & & \phi_{1}\left(u^{*} \cdot u\right)=\phi_{1}, \\
\phi_{2}(w) & =0, & & \phi_{2}\left(w^{*} \cdot w\right)=\phi_{2} .
\end{aligned}
$$

(Actually, Avitzour required also $\phi_{1}$ and $\phi_{2}$ to be faithful, but this hypothesis is easily dispensed with.)

Avitzour's conditions imply simplicity of many reduced free product $\mathrm{C}^{*}$-algebras, but there are plenty of cases where Avitzour's conditions are not satisfied (see $\S 4$ of [9]), yet intuition (or the analogous result for von Neumann algebras, see [5]) suggests the algebra is simple. In this paper we give necessary and sufficient conditions for simplicity of the reduced free product of arbitrary finite dimensional abelian $\mathrm{C}^{*}$-algebras. Stated briefly, if

$$
(\mathfrak{A}, \tau)=\left(A, \tau_{A}\right) *\left(B, \tau_{B}\right),
$$

where $A$ and $B$ are finite dimensional abelian $\mathrm{C}^{*}$-algebras satisfying $\operatorname{dim}(A) \geq 3$ and $\operatorname{dim}(B) \geq 2$ and with faithful tracial states $\tau_{A}$ and $\tau_{B}$, then $\mathfrak{A}$ is simple if and only if whenever $p$ is a minimal projection of $A$ and $q$ is a minimal projection of $B$, we have

$$
\tau_{A}(p)+\tau_{B}(q)<1 .
$$

The necessity of this condition can be seen from [1]. Note that the condition from [5] for the analogous free product of von Neumann algebras to be a factor is (4) but with the strict inequality replaced by $\leq$. In addition, when the free product algebra $\mathfrak{A}$ from (3) is not simple, our analysis allows one to easily find all ideals of $\mathfrak{A}$.

We also show that for every reduced free product $C^{*}$-algebra $\mathfrak{A}$ as in (3), the stable rank of $\mathfrak{A}$ is 1 , regardless of the simplicity of $\mathfrak{A}$. The topological stable rank was invented by M.A. Rieffel [17] in order to study "non-stable" K-theory and as a sort of dimension for $\mathrm{C}^{*}$-algebras. Topological stable rank of $\mathrm{C}^{*}$-algebras was in [13] shown to be equal to the Bass stable rank. The first result about the stable rank of reduced free product $\mathrm{C}^{*}$-algebras is in [9], where it is proved that the free product with respect to traces of $\mathrm{C}^{*}$-algebras $A_{1}$ and $A_{2}$ has stable rank 1 if the Avitzour conditions (2) are satisfied. Hence the present paper's results regarding stable rank are, for a restricted class of $\mathrm{C}^{*}$-algebra reduced free products, a considerable generalization, and they lend support to the plausible conjecture that every reduced free product of $\mathrm{C}^{*}$-algebras with respect to faithful, tracial states has stable rank 1.

In $\S 1$ we state the main results proved in the paper. In $\S 2$ concepts and results essential for the sequel are covered, including some dealing with stable rank, full hereditary subalgebras and free products. In $\S 3$ we prove simplicity and stable rank 1 for free products of two $\mathrm{C}^{*}$-algebras, when one is diffuse in a specific sense. In $\S 4$ the results about the free product of finite dimensional abelian algebras are 
proved, as well as some related results about free products of more general algebras. In $\S 5$, results about free products of abelian $\mathrm{C}^{*}$-algebras with states that are inductive limits of the algebras considered in $\S 4$ are proved. In $\S 6$ we consider free products of infinitely many finite dimensional abelian $\mathrm{C}^{*}$-algebras. Finally, in $\S 7$ we make two conjectures about simplicity of other free product $\mathrm{C}^{*}$-algebras.

\section{Statement of the main Results}

In this section, we state the main results in more detail. Let

$$
\begin{aligned}
\left(A, \tau_{A}\right) & =\underset{\alpha_{1}}{\mathbf{p _ { 1 }}} \oplus \underset{\alpha_{2}}{\mathbf{p _ { 2 }}} \oplus \cdots \oplus \underset{\alpha_{n}}{\mathbf{C}}, \\
\left(B, \tau_{B}\right) & ={\underset{\beta_{1}}{q_{1}}}_{\boldsymbol{\alpha}_{n}}^{q_{\beta_{2}}^{q_{2}}} \oplus \cdots \oplus \underset{\beta_{m}}{\mathbf{q _ { m }}} .
\end{aligned}
$$

This notation means that $n \in \mathbf{N}$, that

$$
A=\underbrace{\mathbf{C} \oplus \cdots \oplus \mathbf{C}}_{n \text { times }}
$$

that $p_{k}$ is the projection

$$
p_{k}=\underbrace{0 \oplus \cdots \oplus 0}_{k-1} \oplus 1 \oplus \underbrace{0 \oplus \cdots \oplus 0}_{n-k}
$$

and that $\tau_{A}$ is the state on $A$ given by $\tau_{A}\left(p_{k}\right)=\alpha_{k}$. We thus need $\alpha_{k} \geq 0$ and $\sum_{1}^{n} \alpha_{k}=1$, and because we want the GNS representation of $\tau_{A}$ to be faithful we will always take $\alpha_{k}>0$. The same considerations apply to $\left(B, \tau_{B}\right)$ in (5).

Theorem 1. Let $\left(A, \tau_{A}\right)$ and $\left(B, \tau_{B}\right)$ be finite dimensional, abelian $C^{*}$-algebras with faithful states as in (5), with $\operatorname{dim}(A) \geq 3$ and $\operatorname{dim}(B) \geq 2$. Let

$$
(\mathfrak{A}, \tau)=\left(A, \tau_{A}\right) *\left(B, \tau_{B}\right)
$$

be the reduced free product of $C^{*}$-algebras. Let

$$
\begin{aligned}
L_{+} & =\left\{(i, j) \mid \alpha_{i}+\beta_{j}>1\right\}, \\
L_{0} & =\left\{(i, j) \mid \alpha_{i}+\beta_{j}=1\right\} .
\end{aligned}
$$

Then the stable rank of $\mathfrak{A}$ is 1 and

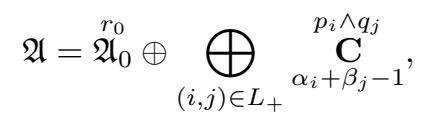

where $p_{i} \wedge q_{j}=\lim _{n \rightarrow \infty}\left(p_{i} q_{j}\right)^{n}$. If $L_{0}$ is empty then $\mathfrak{A}_{0}$ is a simple $C^{*}$-algebra. Otherwise, for every $(i, j) \in L_{0}$, although s.o. $-\lim _{n \rightarrow \infty}\left(p_{i} q_{j}\right)^{n}=0$, there is a unital *-homomorphism $\pi_{(i, j)}: \mathfrak{A}_{0} \rightarrow \mathbf{C}$ such that $\pi_{(i, j)}\left(r_{0} p_{i}\right)=1=\pi_{(i, j)}\left(r_{0} q_{j}\right)$ and

$$
\mathfrak{A}_{00} \stackrel{\text { def }}{=} \bigcap_{(i, j) \in L_{0}} \operatorname{ker} \pi_{(i, j)}
$$

is simple, nonunital and with unique tracial state $\left.\phi\left(r_{0}\right)^{-1} \phi\right|_{\mathfrak{A}_{00}}$.

The notation in (6) means that $\mathfrak{A}=\mathfrak{A}_{0}$ if $L_{+}$is empty, and otherwise

$$
\mathfrak{A}=\mathfrak{A}_{0} \oplus \underbrace{\mathbf{C} \oplus \cdots \oplus \mathbf{C}}_{\left|L_{+}\right| \text {times }} .
$$

In addition, for each $(i, j) \in L_{+}$the corresponding central summand is $\mathbf{C}\left(p_{i} \wedge q_{j}\right)$, and $\tau\left(p_{i} \wedge q_{j}\right)=\alpha_{i}+\beta_{j}-1$. The assertion that s.o. $-\lim _{n \rightarrow \infty}\left(p_{i} q_{j}\right)^{n}=0$ when 
$\alpha_{i}+\beta_{j}=1$ refers to the strong-operator limit in the GNS representation of $\mathfrak{A}$ with respect to $\tau$. Finally, $r_{0}=1-\sum_{(i, j) \in L_{+}} p_{i} \wedge q_{j}$ is the projection which is the unit of $\mathfrak{A}_{0} \oplus 0 \oplus \cdots \oplus 0$.

Analogous results hold for free products of more than two finite dimensional abelian $\mathrm{C}^{*}$-algebras and for free products of direct sums of other abelian $\mathrm{C}^{*-}$ algebras (see Theorems 4.8, 4.9, 5.3 and 6.1).

The following result is used in the proof of Theorem 1 and is also of independent interest.

Theorem 2. Let $A$ and $B$ be unital $C^{*}$-algebras with states $\phi_{A}$, respectively $\phi_{B}$, whose GNS representations are faithful. Let

$$
(\mathfrak{A}, \phi)=\left(A, \phi_{A}\right) *\left(B, \phi_{B}\right) .
$$

Suppose $B \neq \mathbf{C}$ and the centralizer of $\phi_{A}$ has an abelian subalgebra $D \cong C(X)$ containing the unit of $A$ and such that the restriction of $\phi_{A}$ to $D$ is given by an atomless measure on $X$. Then $\mathfrak{A}$ is simple. If $\phi_{A}$ and $\phi_{B}$ are traces, then $\mathfrak{A}$ has stable rank 1 and $\phi$ is the unique tracial state on $\mathfrak{A}$. If one of $\phi_{A}$ and $\phi_{B}$ is not a trace, then $\mathfrak{A}$ has no tracial states.

\section{Preliminaries}

Definition 2.1. Let $A$ be a unital $\mathrm{C}^{*}$-algebra and $\phi$ a state on $A$. Let $B \cong C(X)$ be an abelian $\mathrm{C}^{*}$-subalgebra of $A$ containing the unit of $A$. We say that $\phi$ is diffuse on $B$ if $\left.\phi\right|_{B}$ is given by a measure on $X$ having no atoms. It will usually be clear from the context which state we mean, and then we will speak simply of $B$ being a diffuse abelian subalgebra of $A$.

Given a $\mathrm{C}^{*}$-algebra with state $(A, \phi)$, a Haar unitary (with respect to $\phi$ ) is a unitary element $u \in A$ such that $\phi\left(u^{n}\right)=0$ for every nonzero integer $n$.

Proposition 2.2 [9, 4.1(i)]. Let $B$ be a unital, abelian $C^{*}$-algebra with state $\phi$. Then $\phi$ is diffuse on $B$ if and only if $B$ contains a Haar unitary (with respect to $\phi)$.

Recall that the centralizer of the state $\phi$ is $\{a \in A \mid \forall x \in A \phi(a x)=\phi(x a)\}$. We will often be interested in the situation when the centralizer of $\phi$ contains a Haar unitary.

An ideal of a $\mathrm{C}^{*}$-algebra always means a closed, two-sided ideal.

For unital $\mathrm{C}^{*}$-algebras $A_{1}, A_{2}, \ldots, A_{n}$, it is an obvious fact that $A_{1} \oplus A_{2} \oplus \cdots \oplus A_{n}$ has stable rank 1 if and only if for each $j, A_{j}$ has stable rank 1 . In addition, we will make use of the following results, due to Rieffel.

Proposition $2.3[17,3.3]$. Let $n \in \mathbf{N}$ and let $A$ be a $C^{*}$-algebra. Then $A$ has stable rank 1 if and only if $A \otimes M_{n}(\mathbf{C})$ has stable rank 1 .

The following result follows from [17, 4.4 and 4.11] together with the fact that in a finite dimensional $\mathrm{C}^{*}$-algebra $B$, the left invertible elements are invertible, hence the connected stable rank of $B$ is one.

Proposition 2.4. Let $A$ be a $C^{*}$-algebra with an ideal $J$ such that $A / J$ is finite dimensional. Then $A$ has stable rank 1 if and only if $J$ has stable rank 1.

Recall that a hereditary $\mathrm{C}^{*}$-subalgebra $B$ of a $\mathrm{C}^{*}$-algebra $A$ is said to be full if there is no closed, proper, two-sided ideal of $A$ containing $B$. 
Proposition 2.5. Let $A$ be a $C^{*}$-algebra with countable approximate identity. Take $h \in A, h \geq 0$, and let $B$ be the hereditary subalgebra $\overline{h A h}$ of $A$. Suppose that $B$ is full in $A$. Then

(i) A has stable rank 1 if and only if $B$ has stable rank 1.

(ii) If $B$ has unique tracial state then $A$ has at most one tracial state.

Proof. For (i), note that $B$ has a countable approximate identity for itself because $h$ is strictly positive in $B$ (see [3, p. 327]). Thus, by [3], $A$ and $B$ are stably isomorphic, i.e. $A \otimes \mathcal{K} \cong B \otimes \mathcal{K}$, where $\mathcal{K}$ is the algebra of compact operators on separable Hilbert space. But $[17,3.6]$ states that

$$
\operatorname{sr}(A)=1 \quad \Leftrightarrow \quad \operatorname{sr}(A \otimes \mathcal{K})=1
$$

and similarly for $B$, hence

$$
\operatorname{sr}(A)=1 \quad \Leftrightarrow \quad \operatorname{sr}(B)=1 .
$$

To see (ii), note that $\operatorname{span}\{x h a h y \mid a, x, y \in A\}$ is dense in $A$. If $\tau$ is a tracial state on $A$ then $\tau(x h a h y)=\tau\left(h^{1 / 2} a h y x h^{1 / 2}\right)$ and $h^{1 / 2} a h y x h^{1 / 2} \in B$, so $\tau$ is determined by $\left.\tau\right|_{B}$.

We will say that a positive element $h \in A$ is full if the hereditary subalgebra $\overline{h A h}$ is full in $A$. The following fact is easy to show.

Proposition 2.6. Let $A$ be a $C^{*}$-algebra and let $B$ be a full hereditary $C^{*}$-subalgebra of $A$. Then $A$ is simple if and only if $B$ is simple.

In fact (see [16]), it is well-known that the representation theories of a $\mathrm{C}^{*}$-algebra $A$ and its full hereditary $\mathrm{C}^{*}$-subalgebra $B$ are equivalent.

The reduced free product of two two-dimensional $\mathrm{C}^{*}$-algebras is the most transparent nontrivial free product one can consider. It is understood completely and described in the proposition below. This description is the starting point for our investigation into reduced free products of more general finite dimensional abelian algebras.

Proposition 2.7. Let $1>\alpha \geq \beta \geq \frac{1}{2}$ and let

$$
(\mathfrak{A}, \tau)=(\underset{\alpha}{\mathbf{C}} \oplus \underset{1-\alpha}{\mathbf{C}}) *(\underset{\beta}{\mathbf{C}} \oplus \underset{1-\beta}{\stackrel{1-q}{\mathbf{C}}}) .
$$

If $\alpha>\beta$ then

$$
\mathfrak{A}=\underset{\alpha-\beta}{p \wedge(1-q)} \oplus C\left([a, b], M_{2}(\mathbf{C})\right) \oplus \underset{\alpha+\beta-1}{\mathbf{C}}, \stackrel{p \wedge q}{\mathbf{C}},
$$

for some $0<a<b<1$. Furthermore, in the above picture

$$
\begin{aligned}
& p=1 \oplus\left(\begin{array}{ll}
1 & 0 \\
0 & 0
\end{array}\right) \oplus 1, \\
& q=0 \oplus\left(\begin{array}{cc}
t & \sqrt{t(1-t)} \\
\sqrt{t(1-t)} & 1-t
\end{array}\right) \oplus 1,
\end{aligned}
$$

and the faithful trace $\tau$ is given by the indicated weights on the projections $p \wedge(1-q)$ and $p \wedge q$, together with an atomless measure whose support is $[a, b]$.

If $\alpha=\beta>\frac{1}{2}$ then

$$
\mathfrak{A}=\left\{f:[0, b] \rightarrow M_{2}(\mathbf{C}) \mid f \text { continuous and } f(0) \text { diagonal }\right\} \oplus \underset{\alpha+\beta-1}{\stackrel{p \wedge q}{\mathbf{C}},},
$$


for some $0<b<1$. Furthermore, in the above picture

$$
\begin{aligned}
& p=\left(\begin{array}{ll}
1 & 0 \\
0 & 0
\end{array}\right) \oplus 1, \\
& q=\left(\begin{array}{cc}
t & \sqrt{t(1-t)} \\
\sqrt{t(1-t)} & 1-t
\end{array}\right) \oplus 1,
\end{aligned}
$$

and the faithful trace $\tau$ is given by the indicated weight on the projection $p \wedge q$, together with an atomless measure on $[0, b]$.

If $\alpha=\beta=\frac{1}{2}$ then

$$
\mathfrak{A}=\left\{f:[0,1] \rightarrow M_{2}(\mathbf{C}) \mid f \text { continuous and } f(0) \text { and } f(1) \text { diagonal }\right\} .
$$

Furthermore, in the above picture

$$
\begin{aligned}
& p=\left(\begin{array}{ll}
1 & 0 \\
0 & 0
\end{array}\right), \\
& q=\left(\begin{array}{cc}
t & \sqrt{t(1-t)} \\
\sqrt{t(1-t)} & 1-t
\end{array}\right),
\end{aligned}
$$

and the faithful trace $\tau$ is given by an atomless measure whose support is $[0,1]$.

Proof. Once the traces of $p$ and $q$ are known, the $\mathrm{C}^{*}$-algebra $\mathfrak{A}$ and the trace $\tau$ are determined by $\tau$ composed with the functional calculus of $p q p$. This, in turn, is computed using Voiculescu's multiplicative free convolution [20]. To see this proof in more detail, see [8] or the proof of the analogous result for von Neumann algebras in $[5,1.1]$.

The following proposition is a variation on Theorem 1.2 of [5] and is proved similarly.

Proposition 2.8. Let $A=A_{1} \oplus A_{2}$ be a direct sum of unital $C^{*}$-algebras, write $p=1 \oplus 0 \in A$ and let $\phi_{A}$ be a state on $A$, such that $0<\alpha \stackrel{\text { def }}{=} \phi_{A}(p)<1$. Let $B$ be a unital $C^{*}$-algebra with state $\phi_{B}$ and let $(\mathfrak{A}, \phi)=\left(A, \phi_{A}\right) *\left(B, \phi_{B}\right)$. Let $\mathfrak{A}_{1}$ be the $C^{*}$-subalgebra of $\mathfrak{A}$ generated by $\left(0 \oplus A_{2}\right)+\mathbf{C} p \subseteq A$ together with $B$. We abbreviate this by writing

$$
\begin{aligned}
& (\mathfrak{A}, \phi)=\left(\underset{\alpha}{\stackrel{p}{A}} \oplus \underset{1-\alpha}{1-p} A_{2}^{A}\right) *\left(B, \phi_{B}\right) \\
& \left(\mathfrak{A}_{1},\left.\phi\right|_{\mathfrak{A}_{1}}\right)=\left(\underset{\alpha}{\mathbf{C}} \oplus \underset{1-\alpha}{1-p} A_{2}\right) *\left(B, \phi_{B}\right) .
\end{aligned}
$$

Then $p \mathfrak{A} p$ is generated by $p \mathfrak{A}_{1} p$ and $A_{1} \oplus 0 \subseteq A$, which are free in $\left(p \mathfrak{A} p,\left.\frac{1}{\alpha} \phi\right|_{p \mathfrak{A} p}\right)$.

The next elementary lemma will come in handy.

Lemma 2.9. Let $B$ be a unital $C^{*}$-algebra and $\phi$ a state on $B$ whose GNS representation is faithful. If $|\phi(u)|=1$ for every unitary $u \in B$, then $B=\mathbf{C}$.

Proof. Let the defining embedding $B \hookrightarrow L^{2}(B, \phi)$ be denoted $b \mapsto \hat{b}$. Let $\mathcal{U}(B)$ denote the unitary group of $B$. Whenever $u \in \mathcal{U}(B)$ then $\|\hat{u}\|=1$, but also $|\langle\hat{u}, 1\rangle|=1$, so $\hat{u}=\alpha \hat{1}$ for some $\alpha \in \mathbf{T}$. $\operatorname{But} L^{2}(B, \phi)=\overline{\operatorname{span}}\{\hat{u} \mid u \in \mathcal{U}(B)\}$, so $L^{2}(B, \phi)$ is one-dimensional. This implies $B=\mathbf{C}$. 


\section{WHEN IN THE PRESENCE OF ONE SPREAD THIN}

Let $A$ and $B$ be unital $\mathrm{C}^{*}$-algebras with states $\phi_{A}$ and $\phi_{B}$, respectively, whose GNS representations are faithful, and let

$$
(\mathfrak{A}, \phi)=\left(A, \phi_{A}\right) *\left(B, \phi_{B}\right) .
$$

In this section we will prove that if the centralizer of $\phi_{A}$ contains a unital, diffuse abelian subalgebra then $\mathfrak{A}$ is simple, and if, furthermore, $\phi_{A}$ and $\phi_{B}$ are traces then $\mathfrak{A}$ has stable rank 1 . The diffuse abelian subalgebra is, if you like, "one spread thin." By $[9,4.1(\mathrm{i})]$ (see Proposition 2.2 above) this condition is equivalent to the centralizer of $\phi_{A}$ containing a Haar unitary $u$.

Denote by $\mathfrak{A}_{0}$ the norm dense ${ }^{*}$-subalgebra of $\mathfrak{A}$ that is generated by $A \cup B$. Then, using the standard notation $A^{\circ}=\operatorname{ker} \phi_{A}$ and $B^{\circ}=\operatorname{ker} \phi_{B}$, every element $x$ of $\mathfrak{A}_{0}$ can be written $x=x_{0}+x_{1}$, where $x_{0} \in A$ and

$$
x_{1}=\sum_{j=1}^{N} a_{o}^{(j)} b_{1}^{(j)} a_{1}^{(j)} b_{2}^{(j)} a_{2}^{(j)} \cdots b_{n(j)}^{(j)} a_{n(j)}^{(j)}
$$

with $N \in \mathbf{N}, n(j) \in \mathbf{N}, a_{0}^{(j)}, a_{n(j)}^{(j)} \in A, a_{1}^{(j)}, \ldots, a_{n(j)-1}^{(j)} \in A^{\mathrm{o}}, b_{1}^{(j)}, \ldots, b_{n(j)}^{(j)} \in B^{\mathrm{o}}$. Expressed another way,

$$
x_{1} \in \operatorname{span}(\bigcup_{n=1}^{\infty} A \underbrace{B^{\mathrm{o}} A^{\mathrm{o}} \cdots B^{\mathrm{o}} A^{\mathrm{o}} B^{\mathrm{o}}}_{n \text { times } B^{\circ}} A) .
$$

We begin with a technical lemma. Let $u$ be a Haar unitary in the centralizer of $\left.\phi\right|_{A}$ and write

$$
\begin{gathered}
u^{-\mathbf{N}} \stackrel{\text { def }}{=}\left\{u^{-k} \mid k \in \mathbf{N}\right\}, \\
u^{\mathbf{N}} \stackrel{\text { def }}{=}\left\{u^{k} \mid k \in \mathbf{N}\right\} .
\end{gathered}
$$

Lemma 3.1. With notation as above, suppose that $B \neq \mathbf{C}$ and the centralizer of $\phi_{A}$ contains a Haar unitary $u$. Given $\epsilon>0$ and $x \in \mathfrak{A}$ such that $\phi(x)=0$, there is a unitary, $z \in \mathfrak{A}$ such that $z^{*} x z$ differs in norm by no more than $\epsilon$ from a finite linear combination of elements of

$$
\Theta \stackrel{\text { def }}{=} \bigcup_{n=1}^{\infty} u^{-\mathbf{N}} \underbrace{B^{\mathrm{o}} A^{\mathrm{o}} \cdots B^{\mathrm{o}} A^{\mathrm{o}} B^{\mathrm{o}}}_{n \text { times } B^{\circ}} u^{\mathbf{N}} .
$$

Proof. Since $\mathfrak{A}_{0}$ is dense in $\mathfrak{A}$, we may assume without loss of generality that $x \in \mathfrak{A}_{0}$. By Lemma 2.9 there is a unitary element $v \in B$ such that $0 \leq \phi_{B}(v)<1$. Let $c_{0}=\phi_{B}(v), c_{1}=\sqrt{1-c_{0}^{2}}$ and $y=\left(v-c_{0} 1\right) / c_{1}$, so that 1 and $y$ are orthonormal in $L^{2}\left(B, \phi_{B}\right)$. Let $n, k \in \mathbf{N}$ and let $z=\left(u^{k} v\right)^{n} u^{k}$. Write $x=x_{0}+x_{1}$ with $x_{0} \in A$ and $x_{1}$ as in (7).

We first concern ourselves with $z^{*} x_{1} z$. Writing $x_{1}$ as in (7), let $\eta>0$. Since $\left(u^{p}\right)_{p \in \mathbf{Z}}$ is an orthonormal family in $L^{2}\left(A, \phi_{A}\right)$, we have

$$
\forall a \in A \quad \lim _{p \rightarrow \infty} \phi_{A}\left(a u^{p}\right)=0=\lim _{p \rightarrow \infty} \phi_{A}\left(a u^{-p}\right) .
$$

Using (9), we see that if $k$ is large enough, then for every positive integer $p$ and every $j$ we have $\left|\phi_{A}\left(u^{-p k} a_{0}^{(j)}\right)\right|<\eta$ and $\left|\phi_{A}\left(a_{n(j)}^{(j)} u^{p k}\right)\right|<\eta$. Since $v=c_{0} 1+c_{1} y$, 
we have

$$
a_{n(j)}^{(j)} z=\sum_{\delta_{1}, \ldots, \delta_{n} \in\{0,1\}} c_{\delta_{1}} \cdots c_{\delta_{n}} a_{n(j)}^{(j)} u^{k} y^{\delta_{1}} \cdots u^{k} y^{\delta_{n}} u^{k}
$$

where

$$
\begin{gathered}
y^{-\delta}= \begin{cases}y^{*} & \text { if } \delta=1, \\
1 & \text { if } \delta=0,\end{cases} \\
y^{\delta}= \begin{cases}y & \text { if } \delta=1, \\
1 & \text { if } \delta=0 .\end{cases}
\end{gathered}
$$

If not all the $\delta_{j}$ are zero, then $a_{n(j)}^{(j)} u^{k} y^{\delta_{1}} \cdots u^{k} y^{\delta_{n}} u^{k}$ differs in norm by at most $\eta\|y\|^{m}$ (where $m$ is the number of $1 \leq j \leq n$ for which $\delta_{j}=1$ ) from an element of

$$
\underbrace{A^{\mathrm{o}} y A^{\mathrm{o}} y \cdots A^{\mathrm{o}} y}_{m \text { times } A^{\circ} y} u^{\mathbf{N}} \text {. }
$$

Since $\|y\|<\left(1+c_{0}\right) / c_{1}$, we obtain that $a_{n(j)}^{(j)} z$ differs in norm by at most $c_{0}^{n}\left\|a_{n(j)}^{(j)}\right\|+$ $\left(1+2 c_{0}\right)^{n} \eta$ from an element of

$$
\operatorname{span}(\bigcup_{m=1}^{n} \underbrace{A^{\mathrm{o}} y A^{\mathrm{o}} y \cdots A^{\mathrm{o}} y}_{m \text { times } A^{\circ} y} u^{\mathbf{N}}) \text {. }
$$

Similarly, $z^{*} a_{0}^{(j)}$ differs in norm by at most $c_{0}^{n}\left\|a_{0}^{(j)}\right\|+\left(1+2 c_{0}\right)^{n} \eta$ from an element of

$$
\operatorname{span}(\bigcup_{m=1}^{n} u^{-\mathbf{N}} \underbrace{y^{*} A^{\mathrm{o}} y^{*} A^{\mathrm{o}} \cdots y^{*} A^{\mathrm{o}}}_{m \text { times } y^{*} A^{\circ}}) .
$$

Therefore $z^{*} x_{1} z$ dffers in norm by no more than

$$
\sum_{j=1}^{N}\left(c_{0}^{n}\left\|a_{0}^{(j)}\right\|+\left(1+2 c_{0}\right)^{n} \eta\right)\left\|b_{1}^{(j)} a_{1}^{(j)} b_{2}^{(j)} a_{2}^{(j)} \cdots b_{n(j)}^{(j)}\right\|\left(c_{0}^{n}\left\|a_{n(j)}^{(j)}\right\|+\left(1+2 c_{0}\right)^{n} \eta\right)
$$

from a finite linear combination of elements from $\Theta$. Thus, if $n$ is chosen large enough and then $k$ is chosen large enough, then $z^{*} x_{1} z$ can be made arbitrarily close to a finite linear combination of elements from $\Theta$.

We now examine $z^{*} x_{0} z$. Using again $v=c_{0} 1+c_{1} y$, we have

$$
z^{*} x_{0} z=\sum_{\delta_{1}, \ldots, \delta_{2 n} \in\{0,1\}} c_{\delta_{1}} \cdots c_{\delta_{2 n}} u^{-k} y^{-\delta_{1}} u^{-k} \cdots y^{-\delta_{n}} u^{-k} x_{0} u^{k} y^{\delta_{n+1}} \cdots u^{k} y^{\delta_{2 n}} u^{k} .
$$

We first concentrate on the $2^{n+1}-1$ terms when either $\delta_{1}=\delta_{2}=\cdots=\delta_{n}=0$ or $\delta_{n+1}=\delta_{n+2}=\cdots=\delta_{2 n}=0$. The sum over these terms is equal to

$$
c_{0}^{n}\left(u^{-(n+1) k} x_{0} z+z^{*} x_{0} u^{(n+1) k}-c_{0}^{n} u^{-(n+1) k} x_{0} u^{(n+1) k}\right),
$$


which has norm no greater than $c_{0}^{n}\left\|x_{0}\right\|\left(2+c_{0}^{n}\right)$. This can be made arbitrarily small by choosing $n$ large enough (independently of $k$ ). Each of the remaining $2^{2 n}-2^{n+1}+1$ terms of $(10)$ is of the form

$$
c_{0}^{l} c_{1}^{l^{\prime}} u^{-r_{p} k} y^{*} \cdots u^{-r_{2} k} y^{*} u^{-r_{1} k} y^{*} u^{-r_{0} k} x_{0} u^{s_{0} k} y u^{s_{1} k} y u^{s_{2} k} \cdots y u^{s_{q} k},
$$

where $l^{\prime}, p, q, r_{j}, s_{j}$ are positive integers and $l \geq 0$. Clearly

$$
\phi\left(u^{-r_{0} k} x_{0} u^{s_{0} k}\right)=\phi\left(x_{0} u^{\left(s_{0}-r_{0}\right) k}\right) .
$$

If $r_{0}=s_{0}$ then $\phi\left(u^{-r_{0} k} x_{0} u^{s_{0} k}\right)=\phi\left(x_{0}\right)=0$, and hence the term (11) is an element of $\Theta$. Using (9) we see that by choosing $k$ large enough, each quantity (12) can be made arbitrarily small and hence each of the terms (11) can be made arbitrarily close to an element of $\Theta$. Thus if $n$ is chosen large enough and then $k$ is chosen large enough, then $z^{*} x_{0} z$ can be made arbitrarily close to a finite linear combination of elements from $\Theta$.

Considering the above analyses for $z^{*} x_{1} z$ and $z^{*} x_{0} z$ at the same time, we can choose $n$ large enough and then $k$ large enough so that $z^{*} x z$ is arbitrarily close to a finite linear combination of elements from $\Theta$.

Proposition 3.2. Let $A$ and $B$ be unital $C^{*}$-algebras with states $\phi_{A}$ and $\phi_{B}$, respectively, whose GNS representations are faithful. Let

$$
(\mathfrak{A}, \phi)=\left(A, \phi_{A}\right) *\left(B, \phi_{B}\right) .
$$

Suppose the centralizer of $\phi_{A}$ has a unital, diffuse abelian subalgebra and $B \neq \mathbf{C}$. Then for every $x \in \mathfrak{A}$ and $\epsilon>0$ there are $n \in \mathbf{N}$ and unitaries $z_{1}, \ldots, z_{n} \in \mathfrak{A}$ such that

$$
\left\|\phi(x) 1-\frac{1}{n} \sum_{r=1}^{n} z_{r}^{*} x z_{r}\right\|<\epsilon .
$$

Consequently, $\mathfrak{A}$ is simple. Moreover, if both $\phi_{A}$ and $\phi_{B}$ are traces then $\phi$ is the unique tracial state on $\mathfrak{A}$. If one or both of $\phi_{A}$ and $\phi_{B}$ is not a trace then $\mathfrak{A}$ has no tracial states.

Proof. To prove the existence of $z_{r}$ such that (13) holds, we may without loss of generality assume that $x=x^{*}$ and $\phi(x)=0$, and we may replace $x$ by a unitary conjugate of itself. Let $u$ be a Haar unitary in the centralizer of $\phi_{A}$ Employing Lemma 3.1, we may assume that $x \in \operatorname{span} \Theta,($ see $(8))$. Now we will find $z_{1}, \ldots, z_{5} \in$ $u^{\mathbf{N}}$ such that

$$
\left\|\frac{1}{5} \sum_{r=1}^{5} z_{r}^{*} x z_{r}\right\| \leq \frac{49}{50}\|x\|
$$

These will be found using the technique of [15]. With notation similar to (7), we have

$$
x=\sum_{j=1}^{N} u^{-l_{j}} b_{1}^{(j)} a_{1}^{(j)} \cdots b_{n(j)-1}^{(j)} a_{n(j)-1}^{(j)} b_{n(j)}^{(j)} u^{m_{j}},
$$

for $l_{j}, m_{j} \in \mathbf{N}$. Let $K=\max \left(\bigcup_{j=1}^{N}\left\{l_{j}, m_{j}\right\}\right)+1$. For $1 \leq r \leq 5$, let $z_{r}=$ $u^{r K}$. Let $\mathfrak{M}_{r}$ be the closed subspace of $L^{2}(\mathfrak{A}, \phi)$ spanned by all words of the form $u^{-k} b_{1} a_{1} \cdots b_{n} a_{n}$ with $k \in \mathbf{N},(r-1) K<k \leq r K, n \in \mathbf{N} \cup\{0\}, b_{1}, \ldots, b_{n} \in B^{\circ}$, $a_{1}, \ldots, a_{n-1} \in A^{\mathrm{o}}$ and $a_{n} \in A$. Clearly $p \neq q$ implies $\mathfrak{M}_{p} \perp \mathfrak{M}_{q}$. Since $z_{r}^{*} x z_{r}$ is a 
finite sum of words whose left-most letter lies in $\left\{u^{-k} \mid(r-1) K<k \leq r K\right\}$ and whose right-most letter lies in $\left\{u^{k} \mid(r-1) K<k \leq r K\right\}$, we have

$$
z_{r}^{*} x z_{r}\left(\mathfrak{M}_{r}^{\perp}\right) \subseteq \mathfrak{M}_{r}
$$

Denote by $E_{r}$ the projection from $L^{2}(\mathfrak{A}, \phi)$ onto $\mathfrak{M}_{r}$. Given a unit vector $\xi \in$ $L^{2}(\mathfrak{A}, \phi)$, there is some $1 \leq p \leq 5$ for which $\left\|E_{p} \xi\right\|^{2} \leq \frac{1}{5}$. Thus

$$
\left|\left\langle\frac{1}{5} \sum_{r=1}^{5} z_{r}^{*} x z_{r} \xi, \xi\right\rangle\right| \leq \frac{4}{5}|| x||+\frac{1}{5}\left|\left\langle z_{p}^{*} x z_{p} \xi, \xi\right\rangle\right|,
$$

and since $\left(1-E_{p}\right) z_{p}^{*} x z_{p}\left(1-E_{p}\right)=0$ we have

$$
\left|\left\langle z_{p}^{*} x z_{p} \xi, \xi\right\rangle\right|=\left|\left\langle z_{p}^{*} x z_{p} E_{p} \xi, \xi\right\rangle\right|+\left|\left\langle z_{p}^{*} x z_{p}\left(1-E_{p}\right) \xi, E_{p} \xi\right\rangle\right| \leq 2|| x||\left\|E_{p} \xi\right\| \leq \frac{2}{\sqrt{5}}\|x\| .
$$

Hence

$$
\left|\left\langle\frac{1}{5} \sum_{r=1}^{5} z_{r}^{*} x z_{r} \xi, \xi\right\rangle\right| \leq\left(\frac{4}{5}+\frac{2}{\sqrt{5}}\right)\|x\|<\frac{49}{50}\|x\| .
$$

This implies (14).

To finish the proof of (13), note that the element $\frac{1}{5} \sum_{r=1}^{5} z_{r}^{*} x z_{r}$ obtained above is again in span $\Theta$. Hence, by repeating this process as many times as necessary, for any $\epsilon>0$ there are $n \in \mathbf{N}$ and $z_{1}, \ldots, z_{n} \in u^{\mathbf{N}}$ such that $\left\|\frac{1}{n} \sum_{r=1}^{n} z_{r}^{*} x z_{r}\right\|<\epsilon$.

Now the remaining facts follow by standard arguments of [15] and [2]. Indeed, suppose $\mathcal{I}$ is a nonzero, two-sided, closed ideal of $\mathfrak{A}$. Let $a \in \mathcal{I} \backslash\{0\}$. Since the GNS representation of $\mathfrak{A}$ associated to $\phi$ is faithful, there must be $b \in \mathfrak{A}$ such that $\phi\left(b^{*} a^{*} a b\right) \neq 0$. Then from (13), it follows that $\phi\left(b^{*} a^{*} a b\right) 1 \in \mathcal{I}$; hence $\mathcal{I}=\mathfrak{A}$ and consequently $\mathfrak{A}$ is simple.

The property described at (13) implies that any tracial state on $\mathfrak{A}$ must be equal to $\phi$. If both $\phi_{A}$ and $\phi_{B}$ are traces, then the free product state $\phi$ is also a trace, and is thus the unique tracial state. If one of $\phi_{A}$ and $\phi_{B}$ is not a trace, then neither is $\phi$ a trace; hence $\mathfrak{A}$ has no tracial states.

Lemma 3.3. Let $(\mathfrak{A}, \phi)$ be as in Proposition 3.2. Let $x \in \mathfrak{A}$ and let $\epsilon>0$. Then there are unitaries $z_{1}, z_{2} \in \mathfrak{A}$ such that $\left\|z_{1} x z_{2}-x^{\prime}\right\|<\epsilon$ for some $x^{\prime} \in \operatorname{span} \Theta$, with $\Theta$ as in (8).

Proof. Let $u \in A$ be a Haar unitary in the cetralizer of $\phi_{A}$ and let $v \in B$ a unitary such that $0 \leq \phi_{B}(v)<1$. By Lemma 3.1 there is a unitary $z \in \mathfrak{A}$ such that

$$
\left\|z^{*} x z-\left(\phi(x) 1+x^{\prime \prime}\right)\right\|<\epsilon / 2,
$$

where $x^{\prime \prime} \in \operatorname{span} \Theta$. Writing $v=c_{0} 1+c_{1} y$ as in the proof of Lemma 3.1, we see that $\left(u^{*} v\right)^{p} u^{p}$ differs in norm by no more that $c_{0}^{p}$ from an element of $\operatorname{span} \Theta$. We similarly see that $\left(u^{*} v\right)^{p} x^{\prime \prime} \in \operatorname{span} \Theta$. Let $p$ be so large that $c_{0}^{p}<\epsilon /(2+2|\phi(x)|)$. Let $z_{1}=\left(u^{*} v\right)^{p} z^{*}$ and $z_{2}=z u^{p}$. Then from (15) we have

$$
\left\|z_{1} x z_{2}-\left(\phi(x)\left(u^{*} v\right)^{p} u^{p}+\left(u^{*} v\right)^{p} x^{\prime \prime} u^{p}\right)\right\|<\epsilon / 2
$$

and from the above discussion there is $x^{\prime} \in \operatorname{span} \Theta$ such that

$$
\left\|\phi(x)\left(u^{*} v\right)^{p} u^{p}+\left(u^{*} v\right)^{p} x^{\prime \prime} u^{p}-x^{\prime}\right\|<\epsilon / 2 .
$$

Hence $\left\|z_{1} x z_{2}-x^{\prime}\right\|<\epsilon$.

The proof of the following proposition uses ideas from [9]. 
Proposition 3.4. Let $A$ and $B$ be unital $C^{*}$-algebras with faithful, tracial states $\tau_{A}$ and $\tau_{B}$, respectively. Let

$$
(\mathfrak{A}, \tau)=\left(A, \tau_{A}\right) *\left(B, \tau_{B}\right) .
$$

Suppose $A$ has a unital, diffuse abelian subalgebra and $B \neq \mathbf{C}$. Then $\mathfrak{A}$ has stable rank 1 , i.e. the set of invertible elements of $\mathfrak{A}$ is dense in $\mathfrak{A}$.

Proof. Suppose for contradiction that the set of invertibles in $\mathfrak{A}$, denoted GL( $\mathfrak{A})$, is not dense. Then, by $[18,2.6]$, there is $x \in \mathfrak{A}$ such that $\|x\|=1$ and $\operatorname{dist}(x, \operatorname{GL}(\mathfrak{A}))=$ 1. We must have $\|x\|_{2}<1$, since $\|x\|_{2}=1$ would imply that $x$ is unitary. Let $\epsilon=1-\|x\|_{2}$. Let $u$ be a Haar unitary in $A$ and let $v \in B$ be a unitary such that $0 \leq \tau_{B}(v)<1$. By Lemma 3.3 there are $n \in \mathbf{N}$ and unitaries $z_{1}, z_{2} \in \mathfrak{A}$ such that $\left\|z_{1} x z_{2}-x^{\prime}\right\|<\epsilon / 8$ for some $x^{\prime} \in \operatorname{span} \Psi_{n}$, where

$$
\Psi_{n}=\left\{u^{l} b_{1} a_{1} \cdots b_{k-1} a_{k-1} b_{k} u^{m} \mid k, l, m \in \mathbf{N}, l, m<n, b_{j} \in B^{\circ}, a_{j} \in A^{\circ}\right\} .
$$

Let $p$ be so large that $c_{0}^{p}<\epsilon /(8(\|x\|+\epsilon))$. By writing $v=c_{0} 1+c_{1} y$ as in the proof of Lemma 3.1, we see that

$$
\left\|\left(u^{n} v\right)^{p} u^{n} x^{\prime}-x^{\prime \prime}\right\|<\epsilon / 8
$$

for some $x^{\prime \prime} \in \operatorname{span} \Psi_{n, p}$, where

$$
\Psi_{n, p}=\left\{u^{n l} b_{1} a_{1} \cdots b_{k-1} a_{k-1} b_{k} u^{m} \mid k, l, m \in \mathbf{N}, l<p, m<n, b_{j} \in B^{\mathrm{o}}, a_{j} \in A^{\mathrm{o}}\right\} .
$$

For $q \in \mathbf{N}$ let $E_{q}: A \rightarrow A$ be

$$
E_{q}(a)=\sum_{j=q+1}^{q+n p} u^{j}\left\langle\hat{a},\left(u^{j}\right)^{\wedge}\right\rangle,
$$

where we denote the defining embedding $A \hookrightarrow L^{2}\left(A, \tau_{A}\right)$ by $a \mapsto \hat{a}$.

Since $\lim _{j \rightarrow \infty}\left\langle\hat{a},\left(u^{j}\right)^{\wedge}\right\rangle=0$, we have $\lim _{q \rightarrow \infty}\left\|E_{q}(a)\right\|=0$ for every $a \in A$. Therefore, there is $q$, a positive multiple of $n$, such that $\left\|x^{(3)}-x^{\prime \prime}\right\|<\epsilon / 8$ for some $x^{(3)}$ in span $\Psi_{n, p, q}^{\prime}$, where

$$
\begin{array}{r}
\Psi_{n, p, q}^{\prime}=\left\{u^{n l} b_{1} a_{1} \cdots b_{k-1} a_{k-1} b_{k} u^{m} \mid k, l, m \in \mathbf{N}, l<p, m<n,\right. \\
\left.b_{j} \in B^{\circ}, a_{j} \in A^{\mathrm{o}}, E_{q}\left(a_{j}\right)=0\right\} .
\end{array}
$$

Let $X_{A}$ be a standard orthonormal basis (see $\left.[9, \S 2]\right)$ for $\left(A, \tau_{A}\right)$ containing $\left\{u, u^{2}\right.$, $\left.u^{3}, \ldots, u^{q+n p}\right\}$ and let $X_{B}$ be a standard orthonormal basis for $\left(B, \tau_{B}\right)$. Let $Y=$ $X_{A} * X_{B}$ be the resulting free product standard orthonormal basis for $(\mathfrak{A}, \tau)$. (Note that, by definition, $Y \backslash\{1\}$ is the set of all reduced words in $X_{A} \backslash\{1\}$ and $X_{B} \backslash\{1\}$.) Then there is $x^{(4)} \in \operatorname{span} Y_{n, p, q}^{\prime}$ such that $\left\|u^{q} x^{(3)}-x^{(4)}\right\|<\epsilon / 8$, where $Y_{n, p, q}^{\prime}$ is the subset of $Y$ defined by

$$
\begin{array}{r}
Y_{n, p, q}^{\prime}=\left\{u^{q+n l} b_{1} a_{1} \cdots b_{k-1} a_{k-1} b_{k} u^{m} \mid l, m, k \in \mathbf{N}, l<p, m<n, b_{j} \in X_{B} \backslash\{1\},\right. \\
\left.a_{j} \in X_{A} \backslash\left\{1, u^{q+1}, u^{q+2}, \ldots, u^{q+n p}\right\}\right\} .
\end{array}
$$

Now we see that, since no cancellation occurs when we multiply elements of $Y_{n, p, q}^{\prime}$ (but only " $u$ on $u$ contact"), whenever $w_{1}, \ldots, w_{m} \in Y_{n, p}^{\prime}$ we have

$$
\left\|w_{1} w_{2} \cdots w_{m}\right\|_{2}=\left\|w_{1}\right\|_{2}\left\|w_{2}\right\|_{2} \cdots\left\|w_{m}\right\|_{2} .
$$


Moreover, if $w_{1} w_{2} \cdots w_{m}=w_{1}^{\prime} w_{2}^{\prime} \cdots w_{m}^{\prime}$ for any $m \in \mathbf{N}$ and $w_{j}, w_{j}^{\prime} \in Y_{n, p, q}^{\prime}$, then $w_{1}^{\prime}=w_{1}, w_{2}^{\prime}=w_{2}, \ldots, w_{m}^{\prime}=w_{m}$. The reason for this is that when we take the reduced word of $w_{1} w_{2} \ldots w_{m}$, a letter $u^{k}$ for $q \leq k \leq q+n p$ appears at every boundary where $w_{j}$ touches $w_{j+1}(1 \leq j \leq m-1)$, and nowhere else, and writing $u^{k}=u^{r} u^{q+l n}$ for $l, r \in \mathbf{N}$ and $r \leq n$, we see that $u^{r}$ must have been the last letter in $w_{j}$ and $u^{q+l n}$ must have been the first letter in $w_{j+1}$, so we can recover the list of letters, $w_{1}, w_{2}, \ldots, w_{m}$ from their product. Thus we see that $\left\|\left(x^{(4)}\right)^{m}\right\|_{2}=\left\|x^{(4)}\right\|_{2}^{m}$ for every $m \in \mathbf{N}$, and (see $[9,3.2]) K\left(\left(x^{(4)}\right)^{m}\right)=K\left(x^{(4)}\right)$. Now we argue as in the proof of $[9,3.8]$ to show that the spectral radius of $x^{(4)}$, denoted $r\left(x^{(4)}\right)$, is no greater than $\left\|x^{(4)}\right\|_{2}$. Indeed, let $q$ be the largest block length of the words in the support of $x^{(4)}$, so that, in the notation of $[9,2.2]$,

$$
x^{(4)} \in \operatorname{span} \bigcup_{j=1}^{q} Y_{j} \text {. }
$$

Then, by $[9,3.5]$,

$$
\forall m \in \mathbf{N} \quad\left\|\left(x^{(4)}\right)^{m}\right\| \leq(2 m k+1)^{3 / 2} K\left(x^{(4)}\right)\left\|x^{(4)}\right\|_{2}^{m},
$$

where $K\left(x^{(4)}\right)$ is a constant. Hence

$$
r\left(x^{(4)}\right)=\liminf _{m \rightarrow \infty}\left\|\left(x^{(4)}\right)^{m}\right\|^{1 / m} \leq\left\|x^{(4)}\right\|_{2} .
$$

Therefore $\operatorname{dist}\left(x^{(4)}, \mathrm{GL}(\mathfrak{A})\right) \leq\left\|x^{(4)}\right\|_{2}$. But $\left\|x^{(4)}-u^{k}\left(u^{n} v\right)^{p} u^{n} z_{1} x z_{2}\right\|<\epsilon / 2$, so

$$
\begin{aligned}
\operatorname{dist}(x, \mathrm{GL}(\mathfrak{A})) & =\operatorname{dist}\left(u^{k}\left(u^{n} v\right)^{p} u^{n} z_{1} x z_{2}, \mathrm{GL}(\mathfrak{A})\right) \\
& \leq\left\|x^{(4)}-u^{k}\left(u^{n} v\right)^{p} u^{n} z_{1} x z_{2}\right\|+\left\|x^{(4)}\right\|_{2} \\
& <\epsilon / 2+\left\|x^{(4)}-u^{k}\left(u^{n} v\right)^{p} u^{n} z_{1} x z_{2}\right\|_{2}+\left\|u^{k}\left(u^{n} v\right)^{p} u^{n} z_{1} x z_{2}\right\|_{2} \\
& <\epsilon+\|x\|_{2}=1,
\end{aligned}
$$

contradicting the choice of $x$.

Note that Propositions 3.2 and 3.4 combine to prove Theorem 2.

Proposition 3.5. Let $0<\alpha<1$, let $A$ be a unital $C^{*}$-algebra with state $\phi_{A}$ whose GNS representation is faithful, and let

$$
(\mathfrak{A}, \phi)=(\underset{\alpha}{p} \oplus \underset{1-\alpha}{\stackrel{1-p}{\mathbf{C}}}) *\left(A, \phi_{A}\right) .
$$

Suppose the centralizer of $\phi_{A}$ has a unital, diffuse abelian subalgebra. Then the centralizer of $\left.\phi\right|_{p \mathfrak{A} p}$ has a unital, diffuse abelian subalgebra.

Proof. Let $u$ be a Haar unitary in the centralizer of $\phi_{A}$, let $q=u^{*} p u$, and let $B$ be the $\mathrm{C}^{*}$-algebra generated by $\{1, p, q\}$. Then $p$ and $q$ are free, so

$$
\left(B,\left.\phi\right|_{B}\right)=(\underset{\alpha}{\stackrel{p}{\mathbf{C}}} \oplus \underset{1-\alpha}{\mathbf{1}-p}) *(\underset{\alpha}{\mathbf{C}} \oplus \underset{1-\alpha}{\mathbf{C}}) .
$$

Case I(3.5). $\alpha<1 / 2$. Then by Proposition 2.7 we have for some $0<b<1$ that

$$
B \cong\left\{f:[0, b] \rightarrow M_{2}(\mathbf{C}) \mid f \text { continuous and } f(0) \text { diagonal }\right\} \oplus \underset{1-2 \alpha}{\mathbf{C}},
$$

$p B p \cong C([0, b])$ and $\left.\phi\right|_{p B p}$ is given by an atomless measure on $[0, b]$. Thus $p B p$ is a diffuse abelian subalgebra of the centralizer of $\phi \mid p A p$. 
Case II(3.5). $\alpha=1 / 2$. This is just as in Case I, except now

$$
B \cong\left\{f:[0,1] \rightarrow M_{2}(\mathbf{C}) \mid f \text { continuous and } f(0) \text { and } f(1) \text { diagonal }\right\} .
$$

Case $\operatorname{III}_{\mathbf{n}}(3.5) .(n \in \mathbf{N}) .1-2^{-(n-1)}<\alpha \leq 1-2^{-n}$. We argue by induction on $n$. The case $\mathrm{III}_{1}$ reduces to Cases I and II. Let $n>1$. Then $\alpha>1 / 2$, and by Proposition 2.7 there is some $0<b<1$ such that

$$
\begin{aligned}
B \cong\{f:[0, b] \rightarrow & \left.M_{2}(\mathbf{C}) \mid f \text { continuous and } f(0) \text { diagonal }\right\} \oplus \underset{2 \alpha-1}{p \wedge q}, \\
& (p-p \wedge q) B(p-p \wedge q) \cong C([0, b]),
\end{aligned}
$$

and the restriction of $\phi$ to $(p-p \wedge q) B(p-p \wedge q)$ is given by an atomless measure on $[0, b]$. Hence it will suffice to find a diffuse abelian subalgebra of the centralizer of $\left.\phi\right|_{(p \wedge q) \mathfrak{A}(p \wedge q)}$, because adding it to $(p-p \wedge q) B(p-p \wedge q)$ will give a diffuse abelian subalgebra of the centralizer of $\left.\phi\right|_{p \mathfrak{A} p}$.

We claim that the family $\left\{p, q, u^{2}\right\}$ is $*$-free in $(\mathfrak{A}, \phi)$. Indeed, it suffices to show that every reduced word in $p-\phi(p) 1, q-\phi(q) 1$ and nonzero powers of $u^{2}$ evaluates to zero under $\phi$. However, rewriting each $q-\phi(q) 1$ as $u^{*}(p-\phi(p) 1) u$, we see that each such word is equal to a word in $p-\phi(p) 1$ and nonzero powers of $u$. From the freeness of $p$ and $u$, it follows that this word evaluates to zero under $\phi$. Hence $p \wedge q$ and $u^{2}$ are $*$-free.

Letting $D$ be the $\mathrm{C}^{*}$-algebra generated by $\left\{p \wedge q, u^{2}\right\}$, we have

$$
\left(D,\left.\phi\right|_{D}\right) \cong(\underset{2 \alpha-1}{p \wedge q} \oplus \mathbf{C}) *\left(C^{*}(\mathbf{Z}), \tau_{\mathbf{Z}}\right) .
$$

Since $2 \alpha-1 \leq 1-2^{-(n-1)}$, by the inductive hypothesis there is a diffuse abelian subalgebra of $(p \wedge q) D(p \wedge q)$. As remarked above, this finishes the proof.

Corollary 3.6. Let $A$ be a $C^{*}$-algebra with state $\phi_{A}$ whose GNS representation is faithful, and let $n \in \mathbf{N}, n \geq 2$ and

$$
(\mathfrak{A}, \phi)=\left(\underset{\alpha_{1}}{p_{1}} \oplus \underset{\alpha_{2}}{p_{2}} \oplus \cdots \oplus \underset{\alpha_{n}}{\mathbf{C}}\right) *\left(A, \phi_{A}\right) .
$$

Suppose the centralizer of $\phi_{A}$ has a unital, diffuse abelian subalgebra. Then the centralizer of $\phi$ has a unital, diffuse abelian subalgebra containing $\left\{p_{1}, p_{2}, \ldots, p_{n}\right\}$.

Proof. For each $j$, using Proposition 3.5 and considering the subalgebra of $\mathfrak{A}$ generated by $A \cup\left\{p_{j}\right\}$, we see that the centralizer of $\left.\phi\right|_{p_{j} \mathfrak{A} p_{j}}$ has a diffuse abelian subalgebra $D_{j}$. Then $D_{1}+D_{2}+\cdots+D_{n}$ is the required diffuse abelian subalgebra of the centralizer of $\phi$.

\section{Finite dimensional abelian algebras}

In this section, we examine the reduced free product of (finitely many) finite dimensional abelian $\mathrm{C}^{*}$-algebras. The methods used are reminiscent of [5].

Some words about notation are in order. The natural notation

$$
\left(A, \tau_{A}\right)=\underset{\alpha_{1}}{\mathbf{C}} \oplus \underset{\alpha_{2}}{\stackrel{p_{2}}{\mathbf{C}}} \oplus \cdots \oplus \underset{\alpha_{n}}{\mathbf{C}}
$$


for a finite dimensional abelian $\mathrm{C}^{*}$-algebra and a faithful state was explained just before Theorem 1. Similarly, the notation

$$
\mathfrak{A}={\stackrel{\mathfrak{A}}{\mathfrak{A}_{0}}}_{0} \bigoplus_{k}^{\stackrel{r_{k}}{\mathbf{C}}}
$$

was explained after that theorem. Analogously, we will often write expressions like

$$
(\mathfrak{A}, \phi)=\left(A_{0} \oplus \underset{\alpha_{1}}{p_{1}} \oplus \cdots \oplus \underset{\alpha_{n}}{\mathbf{C}}\right) *\left(B, \phi_{B}\right) .
$$

This will mean that $(\mathfrak{A}, \phi)=\left(A, \phi_{A}\right) *\left(B, \phi_{B}\right)$, where

$$
A=A_{0} \oplus \underbrace{\mathbf{C} \oplus \cdots \oplus \mathbf{C}}_{n \text { times }},
$$

where $A_{0}$ is some $\mathrm{C}^{*}$-algebra, where

$$
p_{k}=\underbrace{0 \oplus \cdots \oplus 0}_{k \text { times }} \oplus 1 \oplus \underbrace{0 \oplus \cdots \oplus 0}_{n-k \text { times }}
$$

and where the state $\phi_{A}$ satisfies $\phi_{A}\left(p_{k}\right)=\alpha_{k}$. In the case of (16) we will always assume that every $\alpha_{k}>0$, that $\sum_{1}^{n} \alpha_{k}<1$, and that the GNS representation of the restriction of $\phi_{A}$ to $A_{0} \oplus 0 \oplus \cdots \oplus 0$ is faithful. Usually, we will also desire that the centralizer of the restriction of $\phi_{A}$ to $A_{0} \oplus 0 \oplus \cdots \oplus 0$ have an abelian subalgebra on which it is diffuse (see Definition 2.1) and whose unit is $1 \oplus 0 \oplus \cdots \oplus 0$. This is conveniently expressed by writing "the centralizer of $\left.\phi\right|_{A_{0}}$ has a unital, diffuse abelian subalgebra."

Lemma 4.1. Let

$$
(\mathfrak{A}, \phi)=\left(A_{0} \oplus \underset{\alpha}{\stackrel{p}{\mathbf{C}}}\right) *\left(\underset{\beta_{1}}{q_{1}} \oplus \underset{\beta_{2}}{q_{2}}\right),
$$

where the centralizer of $\left.\phi\right|_{A_{0}}$ has a unital, diffuse abelian subalgebra. Take $\beta_{1} \geq \beta_{2}$. Then

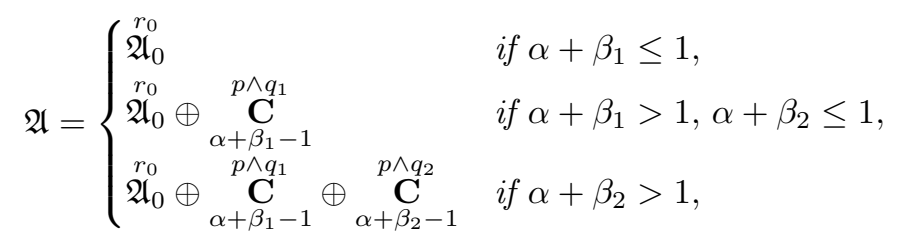

where the centralizer of $\left.\phi\right|_{\mathfrak{A}_{0}}$ has a unital, diffuse abelian subalgebra which contains $r_{0} p$ and a unital, diffuse abelian subalgebra which contains $r_{0} q_{1}$, and where $r_{0} p$ is full in $\mathfrak{A}_{0}$.

If $\left.\phi\right|_{A_{0}}$ is a trace, then the stable rank of $\mathfrak{A}$ is 1.

If $\alpha+\beta_{1} \neq 1$ and $\alpha+\beta_{2} \neq 1$, then $\mathfrak{A}_{0}$ is simple. If, in addition, $\left.\phi\right|_{A_{0}}$ is a trace, then $\left.\phi\left(r_{0}\right)^{-1} \phi\right|_{\mathfrak{A}_{0}}$ is the unique tracial state on $\mathfrak{A}_{0}$ and if $\left.\phi\right|_{A_{0}}$ is not a trace, then $\mathfrak{A}_{0}$ has no tracial states.

Whenever $\alpha+\beta_{i}=1$ for $i \in\{1,2\}$, there is a $*$-homomorphism $\pi_{i}: \mathfrak{A}_{0} \rightarrow \mathbf{C}$ such that $\pi_{i}\left(r_{0} p\right)=1=\pi_{i}\left(q_{i}\right)$.

If $\alpha+\beta_{1}=1$ and $\alpha+\beta_{2}<1$, then $q_{1}$ is full in $\mathfrak{A}_{0}$ and $\operatorname{ker} \pi_{1}$ is simple. If, in addition, $\left.\phi\right|_{A_{0}}$ is a trace, then $\left.\phi\left(r_{0}\right)^{-1} \phi\right|_{\operatorname{ker} \pi_{1}}$ is the unique tracial state on $\operatorname{ker} \pi_{1}$, and if $\left.\phi\right|_{A_{0}}$ is not a trace, then $\operatorname{ker} \pi_{1}$ has no tracial states. 
If $\alpha+\beta_{1}>1$ and $\alpha+\beta_{2}=1$, then $q_{2}$ is full in $\mathfrak{A}_{0}$ and $\operatorname{ker} \pi_{2}$ is simple. If, in addition, $\left.\phi\right|_{A_{0}}$ is a trace, then $\left.\phi\left(r_{0}\right)^{-1} \phi\right|_{\operatorname{ker} \pi_{2}}$ is the unique tracial state on $\operatorname{ker} \pi_{2}$, and if $\left.\phi\right|_{A_{0}}$ is not a trace, then ker $\pi_{2}$ has no tracial states.

If $\alpha+\beta_{1}=1$ and $\alpha+\beta_{2}=1$ (which implies $\alpha=\beta_{1}=\frac{1}{2}$ ), then $q_{1}$ is full in $\operatorname{ker} \pi_{2}$ and $q_{2}$ is full in $\operatorname{ker} \pi_{1}$ and $\left(\operatorname{ker} \pi_{1}\right) \cap\left(\operatorname{ker} \pi_{2}\right)$ is simple. If, in addition, $\left.\phi\right|_{A_{0}}$ is a trace, then $\left.\phi\left(r_{0}\right)^{-1} \phi\right|_{\operatorname{ker} \pi_{1} \cap \operatorname{ker} \pi_{2}}$ is the unique tracial state on $\operatorname{ker} \pi_{1} \cap \operatorname{ker} \pi_{2}$ and if $\left.\phi\right|_{A_{0}}$ is not a trace, then $\operatorname{ker} \pi_{1} \cap \operatorname{ker} \pi_{2}$ has no tracial states.

Proof. Let $\mathfrak{A}_{1}$ be the $\mathrm{C}^{*}$-subalgebra of $\mathfrak{A}$ generated by $\{1, p, q\}$, so

$$
\left(\mathfrak{A}_{1},\left.\phi\right|_{\mathfrak{A}_{1}}\right)=(\underset{1-\alpha}{\mathbf{C}} \oplus \underset{\alpha}{\mathbf{C}}) *\left(\underset{\beta_{1}}{\mathbf{q}_{1}} \oplus \underset{\beta_{2}}{\stackrel{q_{2}}{\mathbf{C}}}\right) .
$$

By Proposition 2.8, $(1-p) \mathfrak{A}(1-p)$ is isomorphic to the free product of $(1-p) \mathfrak{A}_{1}(1-p)$ and $A_{0}$. We use Proposition 2.7 to find $\mathfrak{A}_{1}$. We will also use the fact that $\mathfrak{A}$ is generated by

$$
(1-p) \mathfrak{A}(1-p) \cup(1-p) \mathfrak{A}_{1} p \cup p \mathfrak{A}_{1} p .
$$

Case I(4.1). $\alpha>\beta_{1}$. Then

$$
\mathfrak{A}_{1}=\underset{\alpha+\beta_{2}-1}{\stackrel{p \wedge q_{2}}{\mathbf{C}}} \oplus\left(C([a, b]) \otimes M_{2}(\mathbf{C})\right) \oplus \underset{\alpha+\beta_{1}-1}{\stackrel{p \wedge q_{1}}{\mathbf{C}}},
$$

so $(1-p) \mathfrak{A}(1-p) \cong C([a, b]) * A_{0}$ is simple by Proposition 3.2. Thus

$$
\mathfrak{A} \cong \underset{\alpha+\beta_{2}-1}{\stackrel{p \wedge q_{2}}{\mathbf{C}}} \oplus\left(\left(C([a, b]) * A_{0}\right) \otimes M_{2}(\mathbf{C})\right) \oplus \underset{\alpha+\beta_{1}-1}{\stackrel{p \wedge q_{1}}{\mathbf{C}}} .
$$

Letting $r_{0}=1-p \wedge q_{1}-p \wedge q_{2}$, we then have that

$$
\mathfrak{A}_{0} \stackrel{\text { def }}{=} r_{0} \mathfrak{A}=\left(C([a, b]) * A_{0}\right) \otimes M_{2}(\mathbf{C})
$$

is simple. If $\left.\phi\right|_{A_{0}}$ is a trace, then $(1-p) \mathfrak{A}(1-p)$ has stable rank 1 by Proposition 3.4. Thus also $\mathfrak{A}$ has stable rank 1 . Finally, $r_{0} \mathfrak{A}_{1}$ is clearly in the centralizer of $\phi$. Hence the centralizer of $\left.\phi\right|_{\mathfrak{A}_{0}}$ has a unital, diffuse abelian subalgebra which contains $r_{0} p$ and another which contains $r_{0} q_{1}$.

Case II(4.1). $\alpha=\beta_{1}>\frac{1}{2}$. Then

$$
\mathfrak{A}_{1}=\left\{f:[0, b] \rightarrow M_{2}(\mathbf{C}) \mid f \text { continuous and } f(0) \text { diagonal }\right\} \oplus \underset{\alpha+\beta-1}{\stackrel{p \wedge q_{1}}{\mathbf{C}}},
$$

with $p=\left(\begin{array}{ll}1 & 0 \\ 0 & 0\end{array}\right) \oplus 1$ and $q_{1}=\left(\begin{array}{cc}t & \sqrt{t(1-t)} \\ \sqrt{t(1-t)} & 1-t\end{array}\right) \oplus 1$. Moreover, $p \wedge q_{1}$ is minimal and central in $\mathfrak{A}$ and, by Proposition $3.2,(1-p) \mathfrak{A}(1-p) \cong C([0, b]) * A_{0}$ is simple. Consider the central projection $r_{0}=1-p \wedge q_{1}$, and let $\mathfrak{A}_{0}=r_{0} \mathfrak{A}$. Because $r_{0} \mathfrak{A}_{1}$ is in the centralizer of $\phi$, the centralizer of $\left.\phi\right|_{\mathfrak{A}_{0}}$ has a unital, diffuse abelian subalgebra which contains $r_{0} p$ and and another which contains $r_{0} q_{1}$. Let $\pi_{p \wedge q_{2}}^{(1)}: r_{0} \mathfrak{A}_{1} \rightarrow \mathbf{C}$ be the $*$-homomorphism defined, in the notation of (18), by

$$
\pi_{p \wedge q_{2}}^{(1)}(f)=\text { the }(1,1) \text {-entry of } f(0),
$$

so that $\pi_{p \wedge q_{2}}^{(1)}\left(r_{0} p\right)=1=\pi_{p \wedge q_{2}}^{(1)}\left(q_{2}\right)$. Clearly $r_{0}$ is also central in $\mathfrak{A}$, and the linear span of

$$
\begin{aligned}
r_{0} p \mathfrak{A}_{1} p & +(1-p) \mathfrak{A}(1-p)+(1-p) \mathfrak{A}(1-p) \mathfrak{A}_{1} p \\
& +p \mathfrak{A}_{1}(1-p) \mathfrak{A}(1-p)+p \mathfrak{A}_{1}(1-p) \mathfrak{A}(1-p) \mathfrak{A}_{1} p
\end{aligned}
$$


is dense in $\mathfrak{A}_{0}$. Thus $\pi_{p \wedge q_{2}}^{(1)}$ extends to a $*$-homomorphism $\pi_{p \wedge q_{2}}: \mathfrak{A}_{0} \rightarrow \mathbf{C}$ such that

$$
\begin{aligned}
\operatorname{ker} \pi_{p \wedge q_{2}}^{(1)} & +(1-p) \mathfrak{A}(1-p)+(1-p) \mathfrak{A}(1-p) \mathfrak{A}_{1} p \\
& +p \mathfrak{A}_{1}(1-p) \mathfrak{A}(1-p)+p \mathfrak{A}_{1}(1-p) \mathfrak{A}(1-p) \mathfrak{A}_{1} p
\end{aligned}
$$

spans a dense subset of $\operatorname{ker} \pi_{p \wedge q_{2}}$.

We now show that ker $\pi_{p \wedge q_{2}}$ is simple. Since $(1-p) \mathfrak{A}(1-p)$ is simple, by Proposition 2.6 it will suffice to show that $(1-p) \mathfrak{A}(1-p)$ is full in $\operatorname{ker} \pi_{p \wedge q_{2}}$. But clearly $1-p$ is full in $\operatorname{ker} \pi_{p \wedge q_{2}}^{(1)}$, and $p \mathfrak{A}_{1}(1-p) \subseteq \operatorname{ker} \pi_{p \wedge q_{2}}^{(1)}$. Hence, by the denseness of the span of (19) in $\operatorname{ker} \pi_{p \wedge q_{2}}$, there is no proper ideal of $\operatorname{ker} \pi_{p \wedge q_{2}}$ containing $(1-p) \mathfrak{A}(1-p)$.

Suppose $\left.\phi\right|_{A_{0}}$ is a trace. Then $(1-p) \mathfrak{A}(1-p)$ has stable rank 1 by Proposition 3.4. Since $1-p$ is full in ker $\pi_{p \wedge q_{2}}$, also ker $\pi_{p \wedge q_{2}}$ has stable rank 1 by Proposition 2.5(i). Then $\mathfrak{A}$ has stable rank 1 by Proposition 2.4.

Finally, we show that $q_{2}$ is full in $\mathfrak{A}_{0}$. Suppose $\mathcal{I}$ is an ideal of $\mathfrak{A}_{0}$ containing $q_{2}$. Looking at the ideal of $\mathfrak{A}_{1}$ generated by $q_{2}$, we see that $\mathcal{I}$ contains a nonzero element of $\operatorname{ker} \pi_{p \wedge q_{2}}$, hence by simplicity contains all of $\operatorname{ker} \pi_{p \wedge q_{2}}$. But $q_{2} \notin \operatorname{ker} \pi_{p \wedge q_{2}}$ and $\mathfrak{A}_{0} / \operatorname{ker} \pi_{p \wedge q_{2}}$ is one-dimensional; hence $\mathfrak{A}_{0} \subseteq \mathcal{I}$.

Case $\operatorname{III}(4.1) . \beta_{1}>\alpha>\beta_{2}$. Then

$$
\mathfrak{A}_{1}=\underset{\beta_{1}-\alpha}{q_{1} \wedge(1-p)} \oplus\left(C([a, b]) \otimes M_{2}(\mathbf{C})\right) \oplus \underset{\beta_{1}+\alpha-1}{\stackrel{q_{1} \wedge p}{\mathbf{C}}},
$$

and

$$
(1-p) \mathfrak{A}(1-p) \cong\left(_{\frac{\beta_{1}-\alpha}{1-\alpha}}^{q_{1} \wedge(1-p)} \oplus C([a, b])\right) * A_{0}
$$

is by Proposition 3.2 simple. Let $r_{0}=1-q_{1} \wedge p$ and $\mathfrak{A}_{0}=r_{0} \mathfrak{A}$. Clearly $1-p$ is full in $r_{0} \mathfrak{A}_{1}$ and thus is full in $\mathfrak{A}_{0}$. Hence $\mathfrak{A}_{0}$ is simple. If $D$ is a unital, diffuse abelian subalgebra of the centralizer of $\left.\phi\right|_{A_{0}}$, then $D+\left(p-q_{1} \wedge p\right) \mathfrak{A}_{1}\left(p-q_{1} \wedge p\right)$ is a unital, diffuse abelian subalgebra of the centralizer of $\left.\phi\right|_{\mathfrak{A}_{0}}$ and contains $r_{0} p$. By Proposition 3.5 and considering the $\mathrm{C}^{*}$-subalgebra of $(1-p) \mathfrak{A}(1-p)$ generated by $\left\{q_{1} \wedge(1-p)\right\} \cup A_{0}$, we see that there is a unital, diffuse abelian subalgebra $D$ of the centralizer of $\left.\phi\right|_{\left(q_{1} \wedge(1-p)\right) \mathfrak{A}\left(q_{1} \wedge(1-p)\right)}$. Then

$$
D+q_{2} \mathfrak{A}_{1} q_{2}+\left(q_{1}-q_{1} \wedge(1-p)-q_{1} \wedge p\right) \mathfrak{A}_{1}\left(q_{1}-q_{1} \wedge(1-p)-q_{1} \wedge p\right)
$$

is a unital, diffuse abelian subalgebra of the centralizer of $\left.\phi\right|_{\mathfrak{A}_{0}}$ and contains $r_{0} q_{1}$.

If $\left.\phi\right|_{A_{0}}$ is a trace, then by Proposition $3.4(1-p) \mathfrak{A}(1-p)$ has stable rank 1 . So by Proposition $2.5(\mathrm{i}), \mathfrak{A}_{0}$ has stable rank 1 . We know that

$$
\mathfrak{A}=\mathfrak{A}_{0} \oplus \underset{\alpha+\beta_{1}-1}{\stackrel{p \wedge q_{1}}{\mathbf{C}},}
$$

so $\mathfrak{A}$ has stable rank 1 .

Case IV(4.1). $\frac{1}{2}>\alpha=\beta_{2}$. Then

$$
\mathfrak{A}_{1}=\underset{\beta_{1}-\alpha}{\mathbf{C}} \oplus\left\{f:[a, 1] \rightarrow M_{2}(\mathbf{C}) \mid f \text { continuous and } f(1) \text { diagonal }\right\},
$$


with $0<a<1, p=0 \oplus\left(\begin{array}{ll}1 & 0 \\ 0 & 0\end{array}\right)$ and $q_{1}=1 \oplus\left(\begin{array}{cc}t & \sqrt{t(1-t)} \\ \sqrt{t(1-t)} & 1-t\end{array}\right)$. Thus

$$
(1-p) \mathfrak{A}(1-p) \cong\left(\underset{\frac{\beta_{1}-\alpha}{1-\alpha}}{q_{1} \wedge(1-p)} \oplus C([a, 1])\right) * A_{0}
$$

is by Proposition 3.2 simple. Moreover, if $D$ is a unital, diffuse abelian subalgebra of the centralizer of $\left.\phi\right|_{A_{0}}$, then $D+p \mathfrak{A}_{1} p$ is a unital, diffuse abelian subalgebra of the centralizer of $\phi$ and contains $p$. By Proposition 3.5 and considering the $\mathrm{C}^{*}-$ subalgebra of $(1-p) \mathfrak{A}(1-p)$ generated by $\left\{q_{1} \wedge(1-p)\right\} \cup A_{0}$, we see that there is a unital, diffuse abelian subalgebra $D$ of the centralizer of $\left.\phi\right|_{\left(q_{1} \wedge(1-p)\right) \mathfrak{A}\left(q_{1} \wedge(1-p)\right)}$. Then

$$
D+q_{2} \mathfrak{A}_{1} q_{2}+\left(q_{1}-q_{1} \wedge(1-p)\right) \mathfrak{A}_{1}\left(q_{1}-q_{1} \wedge(1-p)\right)
$$

is a unital, diffuse abelian subalgebra of the centralizer of $\phi$ and contains $q_{1}$.

Let $\pi_{p \wedge q_{1}}^{(1)}: \mathfrak{A}_{1} \rightarrow \mathbf{C}$ be the $*$-homomorphism defined, in the notation of (20), by

$$
\pi_{p \wedge q_{1}}^{(1)}(\lambda \oplus f)=\text { the }(1,1) \text {-entry of } f(1),
$$

so that $\pi_{p \wedge q_{1}}^{(1)}(p)=1=\pi_{p \wedge q_{1}}^{(1)}\left(q_{1}\right)$. Then the linear span of

$$
\begin{aligned}
p \mathfrak{A}_{1} p & +(1-p) \mathfrak{A}(1-p)+(1-p) \mathfrak{A}(1-p) \mathfrak{A}_{1} p \\
& +p \mathfrak{A}_{1}(1-p) \mathfrak{A}(1-p)+p \mathfrak{A}_{1}(1-p) \mathfrak{A}(1-p) \mathfrak{A}_{1} p
\end{aligned}
$$

is clearly dense in $\mathfrak{A}$, so $\pi_{p \wedge q_{1}}^{(1)}$ extends to a $*$-homomorphism $\pi_{p \wedge q_{1}}: \mathfrak{A} \rightarrow \mathbf{C}$ such that

$$
\begin{aligned}
\operatorname{ker} \pi_{p \wedge q_{1}}^{(1)} & +(1-p) \mathfrak{A}(1-p)+(1-p) \mathfrak{A}(1-p) \mathfrak{A}_{1} p \\
& +p \mathfrak{A}_{1}(1-p) \mathfrak{A}(1-p)+p \mathfrak{A}_{1}(1-p) \mathfrak{A}(1-p) \mathfrak{A}_{1} p
\end{aligned}
$$

spans a dense subset of $\operatorname{ker} \pi_{p \wedge q_{1}}$.

As in Case II, since $1-p$ is full in $\operatorname{ker}_{p \wedge q_{1}}^{(1)}$ and since $(1-p) \mathfrak{A}(1-p)$ is simple, it follows that $\operatorname{ker} \pi_{p \wedge q_{1}}$ is simple.

If $\left.\phi\right|_{A_{0}}$ is a trace then by Proposition $3.4,(1-p) \mathfrak{A}(1-p)$ has stable rank 1 . Since $1-p$ is full in ker $\pi_{p \wedge q_{1}}$, also ker $\pi_{p \wedge q_{1}}$ has stable rank 1 by Proposition $2.5(\mathrm{i})$. Thus by Proposition 2.4, $\mathfrak{A}$ has stable rank 1.

Finally, we show that $q_{1}$ is full in $\mathfrak{A}$. Suppose $\mathcal{I}$ is an ideal of $\mathfrak{A}$ containing $q_{1}$. Looking at the ideal of $\mathfrak{A}_{1}$ generated by $q_{1}$, we see that $\mathcal{I}$ contains a nonzero element of ker $\pi_{p \wedge q_{1}}$, hence by simplicity contains all of $\operatorname{ker} \pi_{p \wedge q_{1}}$. But $q_{1} \notin \operatorname{ker} \pi_{p \wedge q_{1}}$ and $\pi_{p \wedge q_{1}}$ is one-dimensional; hence $\mathfrak{A} \subseteq \mathcal{I}$.

Case V(4.1). $\beta_{2}>\alpha$. Then

$$
\mathfrak{A}_{1}=\underset{\beta_{1}-\alpha}{\mathbf{C}} \oplus\left(C([a, b]) \otimes M_{2}(\mathbf{C})\right) \oplus \underset{\beta_{2}-\alpha}{\mathbf{C}}
$$

and

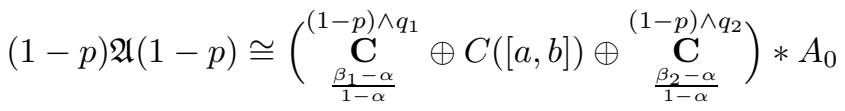

is by Proposition 3.2 simple. Since $1-p$ is full in $\mathfrak{A}_{1}$, is follows that $\mathfrak{A}$ is simple. 
Moreover, if $D$ is a unital, diffuse abelian subalgebra of the centralizer of $\left.\phi\right|_{A_{0}}$, then $D+p \mathfrak{A}_{1} p$ is a unital, diffuse abelian subalgebra of the centralizer of $\phi$ and contains $p$. By Proposition 3.5 and considering the $\mathrm{C}^{*}$-subalgebra of $(1-p) \mathfrak{A}(1-p)$ generated by $\left\{q_{1} \wedge(1-p), q_{2} \wedge(1-p)\right\} \cup A_{0}$, we see that there is a unital, diffuse abelian subalgebra $D_{1}$ and, respectively, $D_{2}$, of the centralizer of $\left.\phi\right|_{\left(q_{1} \wedge(1-p)\right) \mathfrak{A}\left(q_{1} \wedge(1-p)\right)}$ and, respectively, of $\left.\phi\right|_{\left(q_{2} \wedge(1-p)\right) \mathfrak{A}\left(q_{2} \wedge(1-p)\right)}$. Then

$$
\begin{aligned}
D_{1} & +\left(q_{1}-q_{1} \wedge(1-p)\right) \mathfrak{A}_{1}\left(q_{1}-q_{1} \wedge(1-p)\right) \\
& +D_{2}+\left(q_{2}-q_{2} \wedge(1-p)\right) \mathfrak{A}_{1}\left(q_{2}-q_{2} \wedge(1-p)\right)
\end{aligned}
$$

is a unital, diffuse abelian subalgebra of the centralizer of $\phi$ and contains $q_{1}$.

If $\left.\phi\right|_{A_{0}}$ is a trace then by Proposition $3.4(1-p) \mathfrak{A}(1-p)$ has stable rank 1 . Hence by Proposition 2.5(i) also $\mathfrak{A}$ has stable rank 1.

Case $\mathrm{VI}(4.1) . \beta_{1}=\alpha=\frac{1}{2}$. Then

$$
\mathfrak{A}_{1}=\left\{f:[0,1] \rightarrow M_{2}(\mathbf{C}) \mid f \text { continuous, } f(0), f(1) \text { diagonal }\right\},
$$

with $p=\left(\begin{array}{ll}1 & 0 \\ 0 & 0\end{array}\right), q_{1}=\left(\begin{array}{cc}t & \sqrt{t(1-t)} \\ \sqrt{t(1-t)} & 1-t\end{array}\right)$. Thus

$$
(1-p) \mathfrak{A}(1-p) \cong C([0,1]) * A_{0}
$$

is by Proposition 3.2 simple. Moreover, clearly $\mathfrak{A}_{1}$ is in the centralizer of $\phi$ and has unital, diffuse abelian subalgebras containing $p$ and, respectively, $q_{1}$. For $i \in\{1,2\}$ let $\pi_{p \wedge q_{i}}^{(1)}: \mathfrak{A}_{1} \rightarrow \mathbf{C}$ be the $*$-homomorphism defined, in the notation of (22), by

$$
\pi_{p \wedge q_{i}}^{(1)}(f)= \begin{cases}\text { the }(1,1)-\text { entry of } f(1) & \text { if } i=1, \\ \text { the }(1,1)-\text { entry of } f(0) & \text { if } i=2,\end{cases}
$$

so that $\pi_{p \wedge q_{i}}^{(1)}(p)=1=\pi_{p \wedge q_{i}}^{(1)}\left(q_{i}\right)$. Then the linear span of

$$
\begin{aligned}
p \mathfrak{A}_{1} p & +(1-p) \mathfrak{A}(1-p)+(1-p) \mathfrak{A}(1-p) \mathfrak{A}_{1} p \\
& +p \mathfrak{A}_{1}(1-p) \mathfrak{A}(1-p)+p \mathfrak{A}_{1}(1-p) \mathfrak{A}(1-p) \mathfrak{A}_{1} p
\end{aligned}
$$

is clearly dense in $\mathfrak{A}$, so $\pi_{p \wedge q_{i}}^{(1)}$ extends to a $*$-homomorphism $\pi_{p \wedge q_{i}}: \mathfrak{A} \rightarrow \mathbf{C}$ such that

$$
\begin{aligned}
\operatorname{ker} \pi_{p \wedge q_{1}}^{(1)} \cap \operatorname{ker} \pi_{p \wedge q_{2}}^{(1)} & +(1-p) \mathfrak{A}(1-p)+(1-p) \mathfrak{A}(1-p) \mathfrak{A}_{1} p \\
& +p \mathfrak{A}_{1}(1-p) \mathfrak{A}(1-p)+p \mathfrak{A}_{1}(1-p) \mathfrak{A}(1-p) \mathfrak{A}_{1} p
\end{aligned}
$$

spans a dense subset of $\operatorname{ker} \pi_{p \wedge q_{1}} \cap \operatorname{ker} \pi_{p \wedge q_{2}}$.

As in Case II, since $1-p$ is full in $\operatorname{ker}_{p \wedge q_{1}}^{(1)} \cap \operatorname{ker}_{p \wedge q_{2}}^{(1)}$ and since $(1-p) \mathfrak{A}(1-p)$ is simple, it follows that $\operatorname{ker} \pi_{p \wedge q_{1}} \cap \operatorname{ker} \pi_{p \wedge q_{2}}$ is simple.

If $\left.\phi\right|_{A_{0}}$ is a trace, then, by Proposition 3.4, $(1-p) \mathfrak{A}(1-p)$ has stable rank 1 . Since $1-p$ is full in ker $\pi_{p \wedge q_{1}} \cap \operatorname{ker} \pi_{p \wedge q_{2}}$, by Proposition 2.5(i) also ker $\pi_{p \wedge q_{1}} \cap \operatorname{ker} \pi_{p \wedge q_{2}}$ has stable rank 1. Since $\mathfrak{A} /\left(\operatorname{ker} \pi_{p \wedge q_{1}} \cap \operatorname{ker} \pi_{p \wedge q_{2}}\right)$ is two-dimensional, it follows from Proposition 2.4 that $\mathfrak{A}$ has stable rank 1.

We show that $q_{1}$ is full in $\operatorname{ker} \pi_{p \wedge q_{2}}$. Suppose $\mathcal{I}$ is an ideal of $\operatorname{ker} \pi_{p \wedge q_{2}}$ containing $q_{1}$. Multiplying by elements of $\mathfrak{A}_{1}$, we see that $\mathcal{I}$ contains a nonzero element of ker $\pi_{p \wedge q_{1}}$, hence by simplicity contains all of $\operatorname{ker} \pi_{p \wedge q_{1}} \cap \operatorname{ker} \pi_{p \wedge q_{2}}$. But $q_{1} \notin \operatorname{ker} \pi_{p \wedge q_{1}}$ and $\pi_{p \wedge q_{1}}$ is one-dimensional; hence $\operatorname{ker} \pi_{p \wedge q_{2}} \subseteq \mathcal{I}$. The proof that $q_{2}$ is full in $\operatorname{ker} \pi_{p \wedge q_{1}}$ is the same.

We now examine the question of existence and uniqueness of tracial states on the algebras delineated in the statement of the lemma. In all the cases above, it 
follows from Proposition 3.2 that $(1-p) \mathfrak{A}(1-p)$ has tracial states if and only if $\left.\phi\right|_{A_{0}}$ is a trace, and then the free product state gives the unique tracial state on $(1-p) \mathfrak{A}(1-p)$. Moreover, the element $1-p$ is full in the simple algebras under consideration, i.e.

$1-p$ is full in $\mathfrak{A}_{0}$ in Cases I, III and V,

$1-p$ is full in ker $\pi_{p \wedge q_{2}}$ in Case II,

$1-p$ is full in ker $\pi_{p \wedge q_{1}}$ in Case IV,

$1-p$ is full in ker $\pi_{p \wedge q_{1}} \cap \operatorname{ker} \pi_{p \wedge q_{2}}$ in Case VI.

It then follows from Proposition 2.5(ii) that in each of Cases I-VI, the corresponding algebra has tracial states if and only if $\left.\phi\right|_{A_{0}}$ is a trace, and then the restriction of $\phi\left(r_{0}\right)^{-1} \phi$ to this algebra is its unique tracial state. (In the non-unital Cases II, IV and VI, one easily sees that the above normalization gives a state by looking at the subalgebra $r_{0} \mathfrak{A}_{1}$.)

Lemma 4.2. Let

$$
(\mathfrak{A}, \phi)=\left(A_{0} \oplus \underset{\alpha_{1}}{p_{1}} \oplus \underset{\alpha_{2}}{\mathbf{C}}\right) *\left({\stackrel{\beta_{1}}{p_{1}}}_{\beta_{\beta_{2}}}^{\stackrel{q_{2}}{\mathbf{C}}}\right),
$$

where the centralizer of $\left.\phi\right|_{A_{0}}$ has a unital, diffuse abelian subalgebra. Let

$$
\begin{aligned}
L_{+} & =\left\{(i, j) \mid \alpha_{i}+\beta_{j}>1\right\}, \\
L_{0} & =\left\{(i, j) \mid \alpha_{i}+\beta_{j}=1\right\} .
\end{aligned}
$$

Then

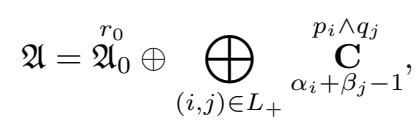

where the centralizer of $\left.\phi\right|_{\mathfrak{A}_{0}}$ has a unital, diffuse abelian subalgebra which contains $r_{0} p_{1}$ and a unital, diffuse abelian subalgebra which contains $r_{0} q_{1}$.

If $\left.\phi\right|_{A_{0}}$ is a trace, then the stable rank of $\mathfrak{A}$ is 1.

If $L_{0}$ is empty, then $\mathfrak{A}_{0}$ is simple. If, in addition, $\left.\phi\right|_{A_{0}}$ is a trace, then $\left.\phi\left(r_{0}\right)^{-1} \phi\right|_{\mathfrak{A}_{0}}$ is the unique tracial state on $\mathfrak{A}_{0}$, and if $\left.\phi\right|_{A_{0}}$ is not a trace, then $\mathfrak{A}_{0}$ has no tracial states.

If $L_{0}$ is not empty, then for every $(i, j) \in L_{0}$ there is a $*$-homomorphism $\pi_{(i, j)}$ : $\mathfrak{A}_{0} \rightarrow \mathbf{C}$ such that $\pi_{(i, j)}\left(r_{0} p_{i}\right)=1=\pi_{(i, j)}\left(r_{0} q_{j}\right)$. Then

(i)

$$
\mathfrak{A}_{00} \stackrel{\text { def }}{=} \bigcap_{(i, j) \in L_{0}} \operatorname{ker} \pi_{(i, j)}
$$

is simple. If $\left.\phi\right|_{A_{0}}$ is a trace then $\left.\phi\left(r_{0}\right)^{-1} \phi\right|_{\mathfrak{A}_{00}}$ is the unique tracial state on $\mathfrak{A}_{00}$, and if $\left.\phi\right|_{A_{0}}$ is not a trace then $\mathfrak{A}_{00}$ has no tracial states.

(ii) For each $i \in\{1,2\}, r_{0} p_{i}$ is full in $\mathfrak{A}_{0} \cap \bigcap_{\substack{\left(i^{\prime}, j\right) \in L_{0} \\ i^{\prime} \neq i}} \operatorname{ker} \pi_{\left(i^{\prime}, j\right)}$.

(iii) For each $j \in\{1,2\}$, $r_{0} q_{j}$ is full in $\mathfrak{A}_{0} \cap \bigcap_{\substack{\left(i, j^{\prime}\right) \in L_{0} \\ j^{\prime} \neq j}} \operatorname{ker} \pi_{\left(i, j^{\prime}\right)}$.

Proof. We will assume that $\alpha_{1} \geq \alpha_{2}$ and $\beta_{1} \geq \beta_{2}$. To prove the lemma in its full generality, we will now be careful to find a unital, diffuse abelian subalgebra of the centralizer of $\left.\phi\right|_{\mathfrak{A}_{0}}$ containing $\left\{r_{0} p_{1}, r_{0} p_{2}\right\}$, not only $r_{0} p_{1}$, and another containing 
$\left\{r_{0} q_{1}, r_{0} q_{2}\right\}$. Let $\mathfrak{A}_{1}$ be the $\mathrm{C}^{*}$-subalgebra of $\mathfrak{A}$ generated by $A_{0}+\mathbf{C}\left(p_{1}+p_{2}\right)$ together with $\left\{q_{1}, q_{2}\right\}$, i.e.

$$
\left(\mathfrak{A}_{1},\left.\phi\right|_{\mathfrak{A}_{1}}\right)=\left(A_{0} \oplus \underset{\alpha_{1}+\alpha_{2}}{\stackrel{p_{1}+p_{2}}{\mathbf{C}}}\right) *\left(\underset{\beta_{1}}{\mathbf{C}} \oplus \underset{\beta_{2}}{\mathbf{q}}\right) .
$$

We find $\mathfrak{A}_{1}$ using Lemma 4.1. Then, by Proposition 2.8

$$
\left(p_{1}+p_{2}\right) \mathfrak{A}\left(p_{1}+p_{2}\right) \cong\left(p_{1}+p_{2}\right) \mathfrak{A}_{1}\left(p_{1}+p_{2}\right) *\left(\underset{\frac{\alpha_{1}}{\alpha_{1}+\alpha_{2}}}{p_{1}} \oplus \underset{\frac{\alpha_{2}}{\alpha_{1}+\alpha_{2}}}{p_{2}}\right)
$$

We consider three cases.

Case I(4.2). $\alpha_{1}+\alpha_{2}+\beta_{1} \leq 1$. Then by Lemma 4.1, the centralizer of $\left.\phi\right|_{\mathfrak{A}_{1}}$ has a unital, diffuse abelian subalgebra $D$ which contains $p_{1}+p_{2}$, and $p_{1}+p_{2}$ is full in $\mathfrak{A}_{1}$. Hence by Proposition $3.2\left(p_{1}+p_{2}\right) \mathfrak{A}\left(p_{1}+p_{2}\right)$ is simple. Also, $p_{1}+p_{2}$ is full in $\mathfrak{A}$, hence $\mathfrak{A}$ is simple. If $\phi_{A_{0}}$ is a trace then, by Proposition 3.4, $\left(p_{1}+p_{2}\right) \mathfrak{A}\left(p_{1}+p_{2}\right)$ has stable rank 1 . Hence by Proposition 2.5(i) so does $\mathfrak{A}$. The application of Lemma 4.1 to (25) yields a unital, diffuse abelian subalgebra of the centralizer of $\phi$ which contains $\left\{q_{1}, q_{2}\right\}$. Applying Corollary 3.6 to (26) shows that the centralizer of $\left.\phi\right|_{\left(p_{1}+p_{2}\right) \mathfrak{A}\left(p_{1}+p_{2}\right)}$ has a unital, diffuse abelian subalgebra $D^{\prime}$ containing $\left\{p_{1}, p_{2}\right\}$. Then $\left(1-p_{1}-p_{2}\right) D\left(1-p_{1}-p_{2}\right)+D^{\prime}$ is a unital, diffuse abelian subalgebra of the centralizer of $\phi$ containing $\left\{p_{1}, p_{2}\right\}$.

Case II(4.2). $\alpha_{1}+\alpha_{2}+\beta_{1}>1$ and $\alpha_{1}+\alpha_{2}+\beta_{2} \leq 1$. Note that this implies

$$
\begin{aligned}
L_{+} & =\left\{(i, 1) \mid \alpha_{i}+\beta_{1}>1\right\}, \\
L_{0} & =\left\{(i, 1) \mid \alpha_{i}+\beta_{1}=1\right\} .
\end{aligned}
$$

Applying Lemma 4.1 to (25) shows that

$$
\mathfrak{A}_{1}=\mathfrak{A}_{1,0}^{r_{1,0}} \oplus \underset{\alpha_{1}+\alpha_{2}+\beta_{1}-1}{\stackrel{\left(p_{1}+p_{2}\right) \wedge q_{1}}{\mathbf{C}}}
$$

where $r_{1,0}=1-\left(p_{1}+p_{2}\right) \wedge q_{1}$, where the centralizer of $\left.\phi\right|_{\mathfrak{A}_{1,0}}$ has a unital, diffuse abelian subalgebra containing $r_{1,0}\left(p_{1}+p_{2}\right)$ and where each of $r_{1,0}\left(p_{1}+p_{2}\right)$ and $q_{2}$ is full in $\mathfrak{A}_{1,0}$. Then

$$
\left(p_{1}+p_{2}\right) \mathfrak{A}_{1}\left(p_{1}+p_{2}\right)=\left(p_{1}+p_{2}\right) \mathfrak{A}_{1,0}\left(p_{1}+p_{2}\right) \oplus \underset{\frac{\left(p_{1}+p_{1}\right) \wedge q_{1}}{\mathbf{C}}}{\frac{\alpha_{1}+\alpha_{2}+\beta_{1}-1}{\alpha_{1}+\alpha_{2}}} .
$$

So, from (26) and Lemma 4.1,

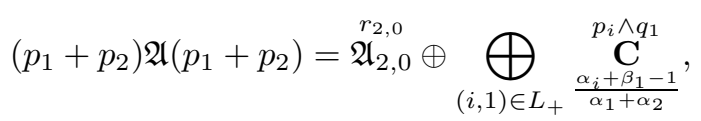

where

$$
r_{2,0}=p_{1}+p_{2}-\sum_{(i, 1) \in L_{+}} p_{i} \wedge q_{1} .
$$

Since $\mathfrak{A}$ is generated by $\left(p_{1}+p_{2}\right) \mathfrak{A}\left(p_{1}+p_{2}\right) \cup \mathfrak{A}_{1}$, we have

$$
\mathfrak{A}=\stackrel{\mathfrak{A}}{0}_{0}^{r_{0}} \oplus \bigoplus_{(i, 1) \in L_{+}} \stackrel{p_{i} \wedge q_{1}}{\mathbf{C}^{+} \beta_{1}-1},
$$


where the linear span of

$$
\begin{aligned}
\mathfrak{A}_{2,0} & +\mathfrak{A}_{2,0} \mathfrak{A}_{1,0}\left(1-p_{1}-p_{2}\right)+\left(1-p_{1}-p_{2}\right) \mathfrak{A}_{1,0} \mathfrak{A}_{2,0} \\
& +\left(1-p_{1}-p_{2}\right) \mathfrak{A}_{1,0} \mathfrak{A}_{2,0} \mathfrak{A}_{1,0}\left(1-p_{1}-p_{2}\right)+\left(1-p_{1}-p_{2}\right) \mathfrak{A}_{1,0}\left(1-p_{1}-p_{2}\right)
\end{aligned}
$$

is dense in $\mathfrak{A}_{0}$. Thus $r_{0}=r_{2,0}+\left(1-p_{1}-p_{2}\right)$. Now the centralizer of $\left.\phi\right|_{\mathfrak{A}_{2,0}}$ has a unital, diffuse abelian subalgebra $D$ which contains $\left\{r_{2,0} p_{1}, r_{2,0} p_{2}\right\}$. Letting $D^{\prime}$ be a unital, diffuse abelian subalgebra of the centralizer of $\left.\phi\right|_{A_{0}}$, it follows that $D+D^{\prime}$ is a unital, diffuse abelian subalgebra of the centralizer of $\left.\phi\right|_{\mathfrak{A}_{0}}$ and contains $\left\{r_{0} p_{1}, r_{0} p_{2}\right\}$.

Now let $D$ be a unital, diffuse abelian subalgebra of the centralizer of $\left.\phi\right|_{\mathfrak{A}_{2,0}}$ containing $r_{2,0}\left(\left(p_{1}+p_{2}\right) \wedge q_{1}\right)$, and $D^{\prime}$ be a unital, diffuse abelian subalgebra of the centralizer of $\left.\phi\right|_{\mathfrak{A}_{1,0}}$ containing $\left\{r_{1,0} q_{1}, q_{2}\right\}$. Then $r_{2,0}\left(\left(p_{1}+p_{2}\right) \wedge q_{1}\right) D+D^{\prime}$ is a unital, diffuse abelian subalgebra of the centralizer of $\left.\phi\right|_{\mathfrak{A}_{0}}$ and contains $\left\{q_{2}, r_{0} q_{1}\right\}$.

Since $r_{1,0}\left(p_{1}+p_{2}\right) \in \mathfrak{A}_{2,0}$ and is full in $\mathfrak{A}_{1,0}$, it follows that $\mathfrak{A}_{2,0}$ is full in $\mathfrak{A}_{0}$. If $L_{0}$ is empty then $\mathfrak{A}_{2,0}$ is simple, hence (by Proposition 2.6) $\mathfrak{A}_{0}$ is also simple.

Otherwise, if $L_{0}$ is nonempty, for every $(i, 1) \in L_{0}$ there is a $*$-homomorphism $\pi_{(i, 1)}^{(2)}: \mathfrak{A}_{2,0} \rightarrow \mathbf{C}$ such that $\pi_{(i, 1)}^{(2)}\left(r_{2,0} p_{i}\right)=1=\pi_{(i, 1)}^{(2)}\left(r_{2,0}\left(\left(p_{1}+p_{2}\right) \wedge q_{1}\right)\right)$. Using the denseness of the span of $(27)$ in $\mathfrak{A}_{0}$, we see that $\pi_{(i, 1)}^{(2)}$ extends to a $*$-homomorphism $\pi_{(i, 1)}: \mathfrak{A}_{0} \rightarrow \mathbf{C}$ such that $\pi_{(i, 1)}\left(r_{0} p_{i}\right)=1=\pi_{(i, 1)}\left(r_{0} q_{1}\right)$ and the linear span of

$$
\begin{aligned}
\operatorname{ker} \pi_{(i, 1)}^{(2)} & +\mathfrak{A}_{2,0} \mathfrak{A}_{1,0}\left(1-p_{1}-p_{2}\right)+\left(1-p_{1}-p_{2}\right) \mathfrak{A}_{1,0} \mathfrak{A}_{2,0} \\
& +\left(1-p_{1}-p_{2}\right) \mathfrak{A}_{1,0} \mathfrak{A}_{2,0} \mathfrak{A}_{1,0}\left(1-p_{1}-p_{2}\right)+\left(1-p_{1}-p_{2}\right) \mathfrak{A}_{1,0}\left(1-p_{1}-p_{2}\right)
\end{aligned}
$$

is dense in $\operatorname{ker} \pi_{(i, 1)}$.

Let

$$
\mathfrak{A}_{2,00}=\mathfrak{A}_{2,0} \cap \bigcap_{(i, 1) \in L_{0}} \operatorname{ker} \pi_{(i, 1)}^{(2)} .
$$

From the application of Lemma $4.1, \mathfrak{A}_{2,00}$ is simple. Since $\mathfrak{A}_{2,00}$ contains $r_{1,0}\left(p_{1}+p_{2}\right)$, which is full in $\mathfrak{A}_{1,0}$, it follows that $\mathfrak{A}_{2,00}$ is full in $\mathfrak{A}_{00}$. Then (by Proposition 2.6), $\mathfrak{A}_{00}$ is simple.

Let $i \in\{1,2\}$. We now show that $r_{0} p_{i}$ is full in

$$
\mathfrak{A}_{0} \cap \bigcap_{\substack{\left(i^{\prime}, 1\right) \in L_{0} \\ i^{\prime} \neq i}} \operatorname{ker} \pi_{\left(i^{\prime}, 1\right)} .
$$

Suppose $\mathcal{I}$ is an ideal of the algebra in (28) containing $r_{0} p_{i}$. Since $r_{2,0} \leq r_{0}$ and (by Lemma 4.1) $r_{2,0} p_{i}$ is full in

$$
\mathfrak{A}_{2,0} \cap \bigcap_{\substack{\left(i^{\prime}, 1\right) \in L_{0} \\ i^{\prime} \neq i}} \operatorname{ker} \pi_{\left(i^{\prime}, 1\right)}^{(2)},
$$

$\mathcal{I}$ must contain the algebra in (29). Hence, arguing as above, $\mathfrak{A}_{1,0} \subseteq \mathcal{I}$. Thus

$$
\begin{aligned}
& \mathfrak{A}_{2,0} \cap \bigcap_{\substack{\left(i^{\prime}, 1\right) \in L_{0} \\
i^{\prime} \neq i}} \operatorname{ker} \pi_{\left(i^{\prime}, 1\right)}^{(2)}+\mathfrak{A}_{2,0} \mathfrak{A}_{1,0}\left(1-p_{1}-p_{2}\right) \\
& \quad+\left(1-p_{1}-p_{2}\right) \mathfrak{A}_{1,0} \mathfrak{A}_{2,0}+\left(1-p_{1}-p_{2}\right) \mathfrak{A}_{1,0} \mathfrak{A}_{2,0} \mathfrak{A}_{1,0}\left(1-p_{1}-p_{2}\right) \subseteq \mathcal{I},
\end{aligned}
$$

proving that the algebra of $(28)$ is contained in $\mathcal{I}$. 
Similarly, since $r_{2,0}\left(\left(p_{1}+p_{2}\right) \wedge q_{1}\right)$ is full in $\mathfrak{A}_{2,0}$, it follows that $r_{0} q_{1}$ is full in $\mathfrak{A}_{0}$.

If $\left.\phi\right|_{A_{0}}$ is a trace, then from Lemma 4.1 we have that $\mathfrak{A}_{2}$ and indeed $\mathfrak{A}_{2,0}$ has stable rank 1. The fullness of $\mathfrak{A}_{2,0}$ in $\mathfrak{A}_{0}$ implies (via Proposition 2.5(i)) that $\mathfrak{A}_{0}$ has stable rank 1 , hence $\mathfrak{A}$ has stable rank 1 .

Finally, concerning existence and uniqueness of traces, from Lemma 4.1 we have that $\mathfrak{A}_{2,00}$ has a tracial state if and only if $\left.\phi\right|_{A_{0}}$ is a trace, and then $\left.\phi\left(r_{2,0}\right)^{-1} \phi\right|_{\mathfrak{A}_{2,00}}$ is its unique tracial state. The same statement for $\mathfrak{A}_{00}$ then follows from fullness of $\mathfrak{A}_{2,00}$ in $\mathfrak{A}_{00}$ and Proposition 2.5(ii). (One can easily check the normalization.)

Case III(4.2). $\alpha_{1}+\alpha_{2}+\beta_{2}>1$. Since $\beta_{2} \leq \frac{1}{2}$ we must have $\frac{1}{2}<\alpha_{1}+\alpha_{2}$. Let $n \in \mathbf{N}$ be least such that $\alpha_{1}+\alpha_{2} \leq \frac{n}{n+1}$. Thus $n \geq 2$. We will proceed by induction on $n$, proving the case $n=2$ and the inductive step simultaneously. Applying Lemma 4.1 to $(25)$, we have

$$
\mathfrak{A}_{1}=\mathfrak{A}_{1,0}^{r_{1,0}} \oplus \underset{\alpha_{1}+\alpha_{2}+\beta_{1}-1}{\mathbf{C}} \oplus \underset{\alpha_{1}+\alpha_{2}+\beta_{2}-1}{\left.\mathbf{C}+p_{2}\right) \wedge q_{1}}
$$

where $r_{1,0}=1-\left(p_{1}+p_{2}\right) \wedge q_{1}-\left(p_{1}+p_{2}\right) \wedge q_{2}$, where the centralizer of $\left.\phi\right|_{\mathfrak{A}_{1,0}}$ has a unital, diffuse abelian subalgebra containing $r_{1,0}\left(p_{1}+p_{2}\right)$ and where $\mathfrak{A}_{1,0}$ is simple. Thus from (26),

$$
\begin{aligned}
\left(p_{1}+p_{2}\right) \mathfrak{A}\left(p_{1}+p_{2}\right) \cong & \left(\left(p_{1}+p_{2}\right) \mathfrak{A}_{1,0}\left(p_{1}+p_{2}\right) \oplus \underset{\frac{\alpha_{1}+\alpha_{2}+\beta_{1}-1}{\alpha_{1}+\alpha_{2}}}{\mathbf{C}} \oplus \underset{\frac{\alpha_{1}+\alpha_{2}+\beta_{2}-1}{\alpha_{1}+\alpha_{2}}}{\mathbf{C}}\right) \\
& *\left(\underset{\frac{\alpha_{1}}{\alpha_{1}+\alpha_{2}}}{\mathbf{C}} \oplus \underset{\frac{\alpha_{2}}{\alpha_{1}+\alpha_{2}}}{\mathbf{C}}\right) .
\end{aligned}
$$

Now since $\frac{n-1}{n}<\alpha_{1}+\alpha_{2}$, we have

$$
\frac{\alpha_{1}+\alpha_{2}+\beta_{1}-1}{\alpha_{1}+\alpha_{2}}+\frac{\alpha_{1}+\alpha_{2}+\beta_{2}-1}{\alpha_{1}+\alpha_{2}}=2-\frac{1}{\alpha_{2}+\alpha_{2}}<\frac{n-2}{n-1} .
$$

The inductive hypothesis (or, when $n \in\{2,3\}$, the previously considered Case I or Case II) applies, and we have, with $L_{+}$as in (24),

$$
\left(p_{1}+p_{2}\right) \mathfrak{A}\left(p_{1}+p_{2}\right)=\mathfrak{A}_{2,0}^{r_{2,0}} \oplus \bigoplus_{(i, j) \in L_{+}} \stackrel{p_{i} \wedge q_{j}}{\stackrel{\alpha_{i}+\beta_{j}-1}{\alpha_{1}+\alpha_{2}}},
$$

where $r_{2,0}=p_{1}+p_{2}-\sum_{(i, j) \in L_{+}} p_{i} \wedge q_{j}$. We obtain that

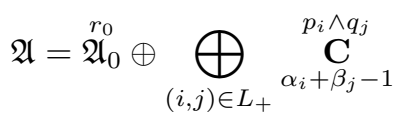

and that the span of $(27)$ is dense in $\mathfrak{A}_{0}$. So $r_{0}=r_{2,0}+\left(1-p_{1}-p_{2}\right)$. Letting $D$ be a unital, diffuse abelian subalgebra of the centralizer of $\phi{\mid \mathfrak{A}_{2,0}}_{\text {containing }}$ $\left\{r_{2,0} p_{1}, r_{2,0} p_{2}\right\}$, and $D^{\prime}$ a unital, diffuse abelian subalgebra of the centralizer of $\left.\phi\right|_{A_{0}}$, we see that $D+D^{\prime}$ is a unital, diffuse abelian subalgebra of the centralizer of $\phi$ and contains $\left\{r_{0} p_{1}, r_{0} p_{2}\right\}$.

Let $D$ be a unital, diffuse abelian subalgebra of the centralizer of $\left.\phi\right|_{\mathfrak{A}_{2,0}}$ that contains $\left\{\left(\left(p_{1}+p_{2}\right) \wedge q_{1}\right) r_{2,0},\left(\left(p_{1}+p_{2}\right) \wedge q_{2}\right) r_{2,0}\right\}$ and let $D^{\prime}$ be a unital, diffuse abelian subalgebra of the centralizer of $\left.\phi\right|_{\mathfrak{A}_{1,0}}$ that contains $\left\{r_{1,0} q_{1}, r_{1,0} q_{2}\right\}$. Then

$$
r_{2,0}\left(\left(p_{1}+p_{2}\right) \wedge q_{1}\right) D+r_{2,0}\left(\left(p_{1}+p_{2}\right) \wedge q_{2}\right) D+D^{\prime}
$$

is a unital, diffuse abelian subalgebra of the centralizer of $\left.\phi\right|_{\mathfrak{A}_{0}}$ and contains $\left\{r_{0} q_{1}\right.$, $\left.r_{0} q_{2}\right\}$. 
Since $r_{1,0}\left(p_{1}+p_{2}\right) \in \mathfrak{A}_{2,0}$ and is full in $\mathfrak{A}_{1,0}$, it follows that $\mathfrak{A}_{2,0}$ is full in $\mathfrak{A}_{0}$. If $L_{0}$, defined in (24), is empty, then $\mathfrak{A}_{2,0}$ is simple; hence (by Proposition 2.6) $\mathfrak{A}_{0}$ is also simple.

If $L_{0}$ is nonempty, then for every $(i, j) \in L_{0}$ there is a $*$-homomorphism $\pi_{(i, j)}^{(2)}$ : $\mathfrak{A}_{2,0} \rightarrow \mathbf{C}$ such that $\pi_{(i, j)}^{(2)}\left(r_{2,0} p_{i}\right)=1=\pi_{(i, j)}^{(2)}\left(r_{2,0}\left(\left(p_{1}+p_{2}\right) \wedge q_{j}\right)\right)$, and this extends to a $*$-homomorphism $\pi_{(i, j)}: \mathfrak{A}_{0} \rightarrow \mathbf{C}$ such that $\pi_{(i, j)}\left(r_{0} p_{i}\right)=1=\pi_{(i, j)}\left(r_{0} q_{j}\right)$ and the linear span of

$$
\begin{aligned}
\operatorname{ker} \pi_{(i, j)}^{(2)} & +\mathfrak{A}_{2,0} \mathfrak{A}_{1,0}\left(1-p_{1}-p_{2}\right)+\left(1-p_{1}-p_{2}\right) \mathfrak{A}_{1,0} \mathfrak{A}_{2,0} \\
& +\left(1-p_{1}-p_{2}\right) \mathfrak{A}_{1,0} \mathfrak{A}_{2,0} \mathfrak{A}_{1,0}\left(1-p_{1}-p_{2}\right)+\left(1-p_{1}-p_{2}\right) \mathfrak{A}_{1,0}\left(1-p_{1}-p_{2}\right)
\end{aligned}
$$

is dense in $\operatorname{ker} \pi_{(i, j)}$.

Let

$$
\mathfrak{A}_{2,00}=\mathfrak{A}_{2,0} \cap \bigcap_{(i, j) \in L_{0}} \operatorname{ker} \pi_{(i, j)}^{(2)} .
$$

From the application of Lemma $4.1, \mathfrak{A}_{2,00}$ is simple. Since $\mathfrak{A}_{2,00}$ contains $r_{1,0}\left(p_{1}+p_{2}\right)$, which is full in $\mathfrak{A}_{1,0}$, it follows that $\mathfrak{A}_{2,00}$ is full in $\mathfrak{A}_{00}$. Then (by Proposition 2.6), $\mathfrak{A}_{00}$ is simple.

Let $i \in\{1,2\}$. We will now show that $r_{0} p_{i}$ is full in

$$
\mathfrak{A}_{0} \cap \bigcap_{\substack{\left(i^{\prime}, j\right) \in L_{0} \\ i^{\prime} \neq i}} \operatorname{ker} \pi_{\left(i^{\prime}, j\right)} .
$$

Suppose $\mathcal{I}$ is an ideal of the algebra in (30) which contains $r_{0} p_{i}$. Since $r_{2,0} \leq r_{0}$ and since $r_{2,0} p_{i}$ is full in

$$
\mathfrak{A}_{2,0} \cap \bigcap_{\substack{\left(i^{\prime}, j\right) \in L_{0} \\ i^{\prime} \neq i}} \operatorname{ker} \pi_{\left(i^{\prime}, j\right)}^{(2)},
$$

this algebra must be contained in $\mathcal{I}$. Then $r_{1,0}\left(p_{1}+p_{2}\right) \in \mathcal{I}$. Since $\mathfrak{A}_{1,0}$ is simple, it is then contained in $\mathcal{I}$. Hence

$$
\begin{aligned}
\mathfrak{A}_{2,0} & \cap \bigcap_{\substack{\left(i^{\prime}, j\right) \in L_{0} \\
i^{\prime} \neq i}} \operatorname{ker} \pi_{\left(i^{\prime}, j\right)}^{(2)}+\mathfrak{A}_{2,0} \mathfrak{A}_{1,0}\left(1-p_{1}-p_{2}\right) \\
& +\left(1-p_{1}-p_{2}\right) \mathfrak{A}_{1,0} \mathfrak{A}_{2,0}+\left(1-p_{1}-p_{2}\right) \mathfrak{A}_{1,0} \mathfrak{A}_{2,0} \mathfrak{A}_{1,0}\left(1-p_{1}-p_{2}\right) \subseteq \mathcal{I},
\end{aligned}
$$

proving that the algebra of $(30)$ is contained in $\mathcal{I}$.

Let $j \in\{1,2\}$. We now show that $r_{0} q_{j}$ is full in

$$
\mathfrak{A}_{0} \cap \bigcap_{\substack{\left(i, j^{\prime}\right) \in L_{0} \\ j^{\prime} \neq j}} \operatorname{ker} \pi_{\left(i, j^{\prime}\right)} .
$$

Suppose $\mathcal{I}$ is an ideal of the algebra in (31) which contains $r_{0} q_{j}$. Since $r_{2,0} \leq r_{0}$ and since $r_{2,0} q_{j}$ is full in

$$
\mathfrak{A}_{2,0} \cap \bigcap_{\substack{\left(i, j^{\prime}\right) \in L_{0} \\ j^{\prime} \neq j}} \operatorname{ker} \pi_{\left(i, j^{\prime}\right)}^{(2)},
$$

this algebra must be contained in $\mathcal{I}$. Then $r_{1,0}\left(p_{1}+p_{2}\right) \in \mathcal{I}$, which as before shows that the algebra (31) is contained in $\mathcal{I}$. 
The required results about the stable rank of $\mathfrak{A}$ and the existence and uniqueness of traces on $\mathfrak{A}_{00}$ follow from the inductive hypothesis because (in the simple case) $\mathfrak{A}_{2,0}$ is full in $\mathfrak{A}_{0}$ or (more generally) $\mathfrak{A}_{2,00}$ is full in $\mathfrak{A}_{00}$.

Lemma 4.3. Let $n \in \mathbf{N}, n \geq 3$ and let

$$
(\mathfrak{A}, \phi)=\left(\underset{\alpha_{1}}{p_{1}} \oplus \cdots \oplus \underset{\alpha_{n}}{\mathbf{C}}\right) *\left(\underset{\beta_{1}}{q_{1}} \oplus \underset{\beta_{2}}{q_{2}}\right) .
$$

Let

$$
\begin{aligned}
L_{+} & =\left\{(i, j) \mid \alpha_{i}+\beta_{j}>1\right\} \\
L_{0} & =\left\{(i, j) \mid \alpha_{i}+\beta_{j}=1\right\} .
\end{aligned}
$$

Then

$$
\mathfrak{A}=\stackrel{\mathfrak{A}}{0}_{0}^{r_{0}} \oplus \bigoplus_{(i, j) \in L_{+}}^{\stackrel{p_{i} \wedge q_{j}}{\mathbf{C}}}
$$

where the centralizer of $\left.\phi\right|_{\mathfrak{A}_{0}}$ has a unital, diffuse abelian subalgebra which contains $r_{0} p_{1}$ and a unital, diffuse abelian subalgebra which contains $r_{0} q_{1}$.

Then the stable rank of $\mathfrak{A}$ is 1 .

If $L_{0}$ is empty, then $\mathfrak{A}_{0}$ is simple and $\left.\phi\left(r_{0}\right)^{-1} \phi\right|_{\mathfrak{A}_{0}}$ is the unique tracial state on $\mathfrak{A}_{0}$.

If $L_{0}$ is not empty, then for every $(i, j) \in L_{0}$ there is a $*$-homomorphism $\pi_{(i, j)}$ : $\mathfrak{A}_{0} \rightarrow \mathbf{C}$ such that $\pi_{(i, j)}\left(r_{0} p_{i}\right)=1=\pi_{(i, j)}\left(r_{0} q_{j}\right)$. Then:

(i)

$$
\mathfrak{A}_{00} \stackrel{\text { def }}{=} \bigcap_{(i, j) \in L_{0}} \operatorname{ker} \pi_{(i, j)}
$$

is simple and $\left.\phi\left(r_{0}\right)^{-1} \phi\right|_{\mathfrak{A}_{00}}$ is the unique tracial state on $\mathfrak{A}_{0}$.

(ii) For each $i \in\{1,2, \ldots, n\}, r_{0} p_{i}$ is full in

$$
\mathfrak{A}_{0} \cap \bigcap_{\substack{\left(i^{\prime}, j\right) \in L_{0} \\ i^{\prime} \neq i}} \operatorname{ker} \pi_{\left(i^{\prime}, j\right)}
$$

(iii) For each $j \in\{1,2\}, r_{0} q_{j}$ is full in

$$
\mathfrak{A}_{0} \cap \bigcap_{\substack{\left.i, j^{\prime}\right) \in L_{0} \\ j^{\prime} \neq j}} \operatorname{ker} \pi_{\left(i, j^{\prime}\right)} .
$$

Proof. We proceed by induction on $n$, proving the initial step $n=3$ and the inductive step simultaneously. Let $\mathfrak{A}_{1}$ be the $\mathrm{C}^{*}$-subalgebra of $\mathfrak{A}$ generated by $\left(\mathbf{C}\left(p_{1}+p_{2}\right)+\mathbf{C} p_{3}+\cdots \mathbf{C} p_{n}\right) \cup\left(\mathbf{C} q_{1}+\mathbf{C} q_{2}\right)$. Thus

$$
\left(\mathfrak{A}_{1},\left.\phi\right|_{\mathfrak{A}_{1}}\right) \cong\left(\underset{\alpha_{1}+\alpha_{2}}{p_{1}+p_{2}} \oplus \underset{\alpha_{3}}{\mathbf{C}} \oplus \cdots \oplus \underset{\alpha_{n}}{p_{3}}\right) *\left(\underset{\beta_{1}}{p_{n}} \oplus \underset{\beta_{2}}{\mathbf{C}}\right)
$$


By the inductive hypothesis when $n>3$ or by Proposition 2.7 when $n=3$, letting

$$
\begin{aligned}
L_{+} & =\left\{(i, j) \mid \alpha_{i}+\beta_{j}>1\right\}, & L_{0} & =\left\{(i, j) \mid \alpha_{i}+\beta_{j}=1\right\}, \\
L_{+}^{(1)} & =\left\{(i, j) \mid i \geq 3, \alpha_{i}+\beta_{j}>1\right\}, & L_{0}^{(1)} & =\left\{(i, j) \mid i \geq 3, \alpha_{i}+\beta_{j}=1\right\}, \\
L_{+}^{\prime} & =\left\{j \mid \alpha_{1}+\alpha_{2}+\beta_{j}>1\right\}, & L_{0}^{\prime} & =\left\{j \mid \alpha_{1}+\alpha_{2}+\beta_{j}=1\right\}, \\
L_{+}^{(2)} & =L_{+} \backslash L_{+}^{(1)}, & L_{0}^{(2)} & =L_{0} \backslash L_{0}^{(1)},
\end{aligned}
$$

we have

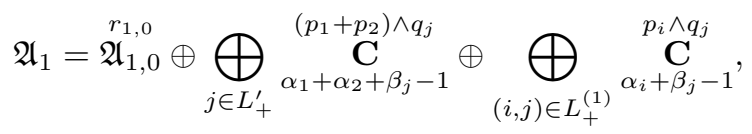

and there is a unital, diffuse abelian subalgebra of the centralizer of $\left.\phi\right|_{\mathfrak{A}_{1,0}}$ containing $r_{1,0}\left(p_{1}+p_{2}\right)$. By Proposition 2.8, $\left(p_{1}+p_{2}\right) \mathfrak{A}\left(p_{1}+p_{2}\right)$ is freely generated by $\left(p_{1}+p_{2}\right) \mathfrak{A}_{1}\left(p_{1}+p_{2}\right)$ and $\left(\mathbf{C} p_{1}+\mathbf{C} p_{2}\right)$, so

$$
\begin{aligned}
& \left(p_{1}+p_{2}\right) \mathfrak{A}\left(p_{1}+p_{2}\right) \\
& \cong\left(\left(p_{1}+p_{2}\right) \mathfrak{A}_{1,0}\left(p_{1}+p_{2}\right) \oplus \bigoplus_{j \in L_{+}^{\prime}}^{\underset{\frac{\alpha_{1}+\alpha_{2}+\beta_{j}-1}{\alpha_{1}+\alpha_{2}}}{\mathbf{C}}} \underset{\left(p_{1}+p_{2}\right) \wedge q_{j}}{\alpha_{1}}\right) *\left(\underset{\frac{\alpha_{1}}{\mathbf{c}_{1}}}{\underset{\alpha_{1}}{\mathbf{C}}} \oplus \underset{\frac{\alpha_{2}}{\alpha_{1}+\alpha_{2}}}{\mathbf{C}}\right) .
\end{aligned}
$$

Noting that $\left|L_{+}^{\prime}\right| \leq 2$, we may use Lemma 4.2 , Lemma 4.1 or results from $\S 3$ to show that

$$
\left(p_{1}+p_{2}\right) \mathfrak{A}\left(p_{1}+p_{2}\right)=\mathfrak{A}_{2,0}^{r_{2,0}} \oplus \bigoplus_{(i, j) \in L_{+}^{(2)}} \underset{\alpha_{i}+\beta_{j}-1}{\mathbf{p}_{i} \wedge q_{j}}
$$

Hence

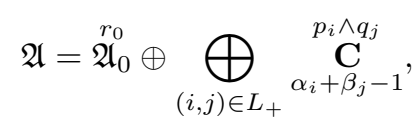

where $r_{0}=r_{2,0}+r_{1,0}\left(1-p_{1}-p_{2}\right)$ and the linear span of the set in (27) is dense in $\mathfrak{A}_{0}$.

The inductive hypothesis (or Proposition 2.7) yields for every $(i, j) \in L_{0}^{(1)}$ a *-homomorphism $\pi_{(i, j)}^{(1)}: \mathfrak{A}_{1,0} \rightarrow \mathbf{C}$ such that $\pi_{(i, j)}^{(1)}\left(r_{1,0} p_{i}\right)=1=\pi_{(i, j)}^{(1)}\left(r_{1,0} q_{j}\right)$. Moreover, for every $(i, j) \in L_{0}^{(2)}$ we have a $*$-homomorphism $\pi_{(i, j)}^{(2)}: \mathfrak{A}_{2,0} \rightarrow \mathbf{C}$ such that $\pi_{(i, j)}^{(2)}\left(r_{2,0} p_{i}\right)=1=\pi_{(i, j)}^{(2)}\left(r_{2,0} q_{j}\right)$. Looking at $(27)$, one easily sees that each of these $*$-homomorphisms can be uniquely extended to a $*$-homomorphism $\pi_{(i, j)}: \mathfrak{A}_{0} \rightarrow \mathbf{C}$ so that $\pi_{(i, j)}\left(r_{0} p_{i}\right)=1=\pi_{(i, j)}\left(r_{0} q_{j}\right)$.

Let

$$
\begin{aligned}
\mathfrak{A}_{1,00} & =\mathfrak{A}_{1,0} \cap \bigcap_{(i, j) \in L_{0}^{(1)}} \operatorname{ker} \pi_{(i, j)}^{(1)}, \\
\mathfrak{A}_{2,00} & =\mathfrak{A}_{2,0} \cap \bigcap_{(i, j) \in L_{0}^{(2)}} \operatorname{ker} \pi_{(i, j)}^{(2)} .
\end{aligned}
$$


Since $r_{1,0}\left(p_{1}+p_{2}\right) \in \mathfrak{A}_{1,00} \cap \mathfrak{A}_{2,00}$, we see from the denseness of the span of (27) in $\mathfrak{A}_{0}$ that the linear span of

$$
\begin{aligned}
\mathfrak{A}_{2,0} & +\mathfrak{A}_{2,00} \mathfrak{A}_{1,00}\left(1-p_{1}-p_{2}\right)+\left(1-p_{1}-p_{2}\right) \mathfrak{A}_{1,00} \mathfrak{A}_{2,00} \\
& +\left(1-p_{1}-p_{2}\right) \mathfrak{A}_{1,00} \mathfrak{A}_{2,00} \mathfrak{A}_{1,00}\left(1-p_{1}-p_{2}\right)+\left(1-p_{1}-p_{2}\right) \mathfrak{A}_{1,0}\left(1-p_{1}-p_{2}\right)
\end{aligned}
$$

is dense in $\mathfrak{A}_{0}$, and hence the linear span of

$$
\begin{aligned}
\mathfrak{A}_{2,00} & +\mathfrak{A}_{2,00} \mathfrak{A}_{1,00}\left(1-p_{1}-p_{2}\right)+\left(1-p_{1}-p_{2}\right) \mathfrak{A}_{1,00} \mathfrak{A}_{2,00} \\
& +\left(1-p_{1}-p_{2}\right) \mathfrak{A}_{1,00} \mathfrak{A}_{2,00} \mathfrak{A}_{1,00}\left(1-p_{1}-p_{2}\right)+\left(1-p_{1}-p_{2}\right) \mathfrak{A}_{1,00}\left(1-p_{1}-p_{2}\right)
\end{aligned}
$$

is dense in $\mathfrak{A}_{00}$. Note that $r_{1,0}\left(p_{1}+p_{2}\right)$ is full in $\mathfrak{A}_{1,00}$. Since $r_{1,0}\left(p_{1}+p_{2}\right) \in \mathfrak{A}_{2,00}$, this implies that $\mathfrak{A}_{2,00}$ is full in $\mathfrak{A}_{00}$. Since $\mathfrak{A}_{2,00}$ is simple, it follows from Proposition 2.6 that $\mathfrak{A}_{00}$ is simple. (This also shows that $\mathfrak{A}_{0}$ is simple when $L_{0}=\emptyset$.)

Let us now prove part (ii). If $i \in\{1,2\}$ then the linear span of

$$
\begin{aligned}
& \left(\mathfrak{A}_{2,0} \cap \bigcap_{\substack{\left(i^{\prime}, j\right) \in L_{0}^{(2)} \\
i^{\prime} \neq i}} \operatorname{ker}_{\left(i^{\prime}, j\right)}^{(2)}\right)+\mathfrak{A}_{2,00} \mathfrak{A}_{1,00}\left(1-p_{1}-p_{2}\right)+\left(1-p_{1}-p_{2}\right) \mathfrak{A}_{1,00} \mathfrak{A}_{2,00} \\
& \quad+\left(1-p_{1}-p_{2}\right) \mathfrak{A}_{1,00} \mathfrak{A}_{2,00} \mathfrak{A}_{1,00}\left(1-p_{1}-p_{2}\right)+\left(1-p_{1}-p_{2}\right) \mathfrak{A}_{1,00}\left(1-p_{1}-p_{2}\right)
\end{aligned}
$$

is dense in (33). But $r_{2,0} p_{i}$ is full in

$$
\mathfrak{A}_{2,0} \cap \bigcap_{\substack{\left(i^{\prime}, j\right) \in L_{0}^{(2)} \\ i^{\prime} \neq i}} \operatorname{ker}_{\left(i^{\prime}, j\right)}^{(2)} .
$$

Since this latter algebra contains $r_{1,0}\left(p_{1}+p_{2}\right)$, which is full in $\mathfrak{A}_{1,00}$, it then follows that $r_{0} p_{i}$ is full in the algebra (33). Now take $i \in\{3,4, \ldots, n\}$. For $j \in L_{0}^{\prime}$ let $\pi_{(0, j)}^{(1)}: \mathfrak{A}_{1,0} \rightarrow \mathbf{C}$ be the $*$-homomorphism such that $\pi_{(0, j)}^{(1)}\left(p_{1}+p_{2}\right)=1=\pi_{(0, j)}^{(1)}\left(q_{j}\right)$. We have that $r_{1,0} p_{i}$ is full in

$$
\mathfrak{A}_{1,0} \cap \bigcap_{j \in L_{0}^{\prime}} \operatorname{ker} \pi_{(0, j)}^{(1)} \cap \bigcap_{\substack{\left(i^{\prime}, j\right) \in L_{0}^{(1)} \\ i^{\prime} \neq i}} \operatorname{ker} \pi_{\left(i^{\prime}, j\right)}^{(1)},
$$

which in turn contains $\left(1-p_{1}-p_{2}\right) \mathfrak{A}_{1,0}\left(p_{1}+p_{2}\right)$ and

$$
\left(1-p_{1}-p_{2}\right)\left(\mathfrak{A}_{1,0} \cap \bigcap_{\substack{\left(i^{\prime}, j\right) \in L_{0}^{(1)} \\ i^{\prime} \neq i}} \operatorname{ker} \pi_{\left(i^{\prime}, j\right)}^{(1)}\right)\left(1-p_{1}-p_{2}\right) .
$$

But $\left(p_{1}+p_{2}\right)\left(\bigcap_{j \in L_{0}^{\prime}} \operatorname{ker} \pi_{(0, j)}^{(1)}\right)\left(p_{1}+p_{2}\right)$ meets $\mathfrak{A}_{2,00}$, which is simple. Hence any ideal of the algebra (34) which contains $r_{0} p_{i}$ must also contain

$$
\begin{aligned}
\mathfrak{A}_{2,00} & +\mathfrak{A}_{2,00} \mathfrak{A}_{1,00}\left(1-p_{1}-p_{2}\right)+\left(1-p_{1}-p_{2}\right) \mathfrak{A}_{1,00} \mathfrak{A}_{2,00} \\
& +\left(1-p_{1}-p_{2}\right) \mathfrak{A}_{1,00} \mathfrak{A}_{2,00} \mathfrak{A}_{1,00}\left(1-p_{1}-p_{2}\right) \\
& +\left(1-p_{1}-p_{2}\right)\left(\mathfrak{A}_{1,0} \cap \bigcap_{\substack{\left(i^{\prime}, j\right) \in L_{0}^{(1)} \\
i^{\prime} \neq i}} \operatorname{ker} \pi_{\left(i^{\prime}, j\right)}^{(1)}\right)\left(1-p_{1}-p_{2}\right),
\end{aligned}
$$

which is dense in the algebra (34). This shows that $r_{0} p_{i}$ is full in (34). 
We now prove part (iii). We have that $r_{1,0} q_{j}$ is full in

$$
\mathfrak{A}_{1,0} \cap \bigcap_{\substack{j^{\prime} \in L_{0}^{\prime} \\ j^{\prime} \neq j}} \operatorname{ker} \pi_{\left(0, j^{\prime}\right)}^{(1)} \cap \bigcap_{\substack{\left(i, j^{\prime}\right) \in L_{0}^{(1)} \\ j^{\prime} \neq j}} \operatorname{ker} \pi_{\left(i, j^{\prime}\right)}^{(1)},
$$

which in turn contains $\left(1-p_{1}-p_{2}\right) \mathfrak{A}_{1,0}\left(p_{1}+p_{2}\right)$ and

$$
\left(1-p_{1}-p_{2}\right)\left(\mathfrak{A}_{1,0} \cap \bigcap_{\substack{\left.i, j^{\prime}\right) \in L_{0}^{(1)} \\ j^{\prime} \neq j}} \operatorname{ker} \pi_{\left(i, j^{\prime}\right)}^{(1)}\right)\left(1-p_{1}-p_{2}\right) .
$$

If $\exists i$ such that $(i, j) \in L_{0}^{(2)}$ then $\alpha_{1}+\alpha_{2}+\beta_{j}>1$, so $\left(p_{1}+p_{2}\right) \wedge q_{j} \neq 0$ and $r_{2,0}\left(\left(p_{1}+p_{2}\right) \wedge q_{j}\right)$ is full in

$$
\mathfrak{A}_{2,0} \cap \bigcap_{\substack{\left(i, j^{\prime}\right) \in L_{0}^{(2)} \\ j^{\prime} \neq j}} \operatorname{ker} \pi_{\left(i, j^{\prime}\right)}^{(2)} .
$$

Hence any ideal of the algebra (34) that contains $r_{0} q_{j}$ must contain

$$
\begin{gathered}
\left(\mathfrak{A}_{2,0} \cap \bigcap_{\substack{\left(i, j^{\prime}\right) \in L_{0}^{(2)} \\
j^{\prime} \neq j}} \operatorname{ker}_{\left(i, j^{\prime}\right)}^{(2)}\right)+\mathfrak{A}_{2,00} \mathfrak{A}_{1,00}\left(1-p_{1}-p_{2}\right)+\left(1-p_{1}-p_{2}\right) \mathfrak{A}_{1,00} \mathfrak{A}_{2,00} \\
+\left(1-p_{1}-p_{2}\right) \mathfrak{A}_{1,00} \mathfrak{A}_{2,00} \mathfrak{A}_{1,00}\left(1-p_{1}-p_{2}\right) \\
+\left(1-p_{1}-p_{2}\right)\left(\mathfrak{A}_{1,0} \cap \bigcap_{\substack{\left(i, j^{\prime}\right) \in L_{0}^{(1)} \\
j^{\prime} \neq j}} \operatorname{ker}_{\left(i, j^{\prime}\right)}^{(1)}\right)\left(1-p_{1}-p_{2}\right)
\end{gathered}
$$

whose span is dense in (34). On the other hand, if there is no $i$ such that $(i, j) \in L_{0}^{(2)}$ then the algebra (39) is $\mathfrak{A}_{2,00}$, which is simple. By a dimension argument, the algebra (38) meets $\mathfrak{A}_{2,00}$. Hence any ideal of the algebra (34) that contains $r_{0} q_{j}$ must contain

$$
\begin{aligned}
\mathfrak{A}_{2,00} & +\mathfrak{A}_{2,00} \mathfrak{A}_{1,00}\left(1-p_{1}-p_{2}\right)+\left(1-p_{1}-p_{2}\right) \mathfrak{A}_{1,00} \mathfrak{A}_{2,00} \\
& +\left(1-p_{1}-p_{2}\right) \mathfrak{A}_{1,00} \mathfrak{A}_{2,00} \mathfrak{A}_{1,00}\left(1-p_{1}-p_{2}\right) \\
& +\left(1-p_{1}-p_{2}\right)\left(\mathfrak{A}_{1,0} \cap \bigcap_{\substack{\left.i, j^{\prime}\right) \in L_{0}^{(1)} \\
j^{\prime} \neq j}} \operatorname{ker}_{\left(i, j^{\prime}\right)}^{(1)}\right)\left(1-p_{1}-p_{2}\right),
\end{aligned}
$$

whose span is dense in (34). Thus $r_{0} q_{j}$ is full in (34).

The required results about stable rank and uniqueness of the trace follow as in previous lemmas from the fact that $\mathfrak{A}_{2,00}$ is full in $\mathfrak{A}_{00}$.

Lemma 4.4. Let $n \in \mathbf{N} \cup\{0\}$ and let

$$
(\mathfrak{A}, \phi)=\left(A_{0} \oplus \underset{\alpha_{1}}{p_{1}} \oplus \cdots \oplus \underset{\alpha_{n}}{\mathbf{C}}\right) *\left({\stackrel{p_{n}}{\mathbf{C}}}_{\beta_{1}}^{q_{1}} \underset{\beta_{2}}{q_{2}}\right),
$$


where the centralizer of $\left.\phi\right|_{A_{0}}$ has a unital, diffuse abelian subalgebra. Let $L_{+}$and $L_{0}$ be as in (32). Then

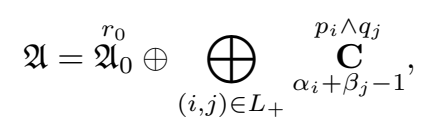

where the centralizer of $\left.\phi\right|_{\mathfrak{A}_{0}}$ has a unital, diffuse abelian subalgebra which contains $r_{0} p_{1}$ and a unital, diffuse abelian subalgebra which contains $r_{0} q_{1}$.

If $\left.\phi\right|_{A_{0}}$ is a trace then the stable rank of $\mathfrak{A}$ is 1.

If $L_{0}$ is empty then $\mathfrak{A}_{0}$ is simple. If, in addition, $\left.\phi\right|_{A_{0}}$ is a trace then $\left.\phi\left(r_{0}\right)^{-1} \phi\right|_{\mathfrak{A}_{0}}$ is the unique tracial state on $\mathfrak{A}_{0}$, and if $\left.\phi\right|_{A_{0}}$ is not a trace then $\mathfrak{A}_{0}$ has no tracial states.

If $L_{0}$ is not empty, then for every $(i, j) \in L_{0}$ there is a $*$-homomorphism $\pi_{(i, j)}$ : $\mathfrak{A}_{0} \rightarrow \mathbf{C}$ such that $\pi_{(i, j)}\left(r_{0} p_{i}\right)=1=\pi_{(i, j)}\left(r_{0} q_{j}\right)$. Then:

(i)

$$
\mathfrak{A}_{00} \stackrel{\text { def }}{=} \bigcap_{(i, j) \in L_{0}} \operatorname{ker} \pi_{(i, j)}
$$

is simple. If $\left.\phi\right|_{A_{0}}$ is a trace then $\left.\phi\left(r_{0}\right)^{-1} \phi\right|_{\mathfrak{A}_{00}}$ is the unique tracial state on $\mathfrak{A}_{0}$, and if $\left.\phi\right|_{A_{0}}$ is not a trace then $\mathfrak{A}_{00}$ has no tracial states.

(ii) For each $i \in\{1,2, \ldots, n\}, r_{0} p_{i}$ is full in

$$
\mathfrak{A}_{0} \cap \bigcap_{\substack{\left(i^{\prime}, j\right) \in L_{0} \\ i^{\prime} \neq i}} \operatorname{ker} \pi_{\left(i^{\prime}, j\right)} .
$$

(iii) For each $j \in\{1,2\}, r_{0} q_{j}$ is full in

$$
\mathfrak{A}_{0} \cap \bigcap_{\substack{\left(i, j^{\prime}\right) \in L_{0} \\ j^{\prime} \neq j}} \operatorname{ker} \pi_{\left(i, j^{\prime}\right)} .
$$

Proof. The cases $n=0,1,2$ have been already proved. Let $p_{0}=1-\sum_{1}^{n} p_{j}$ and let $\mathfrak{A}_{1}$ be the $\mathrm{C}^{*}$-subalgebra of $\mathfrak{A}$ generated by $\left(\mathbf{C} p_{0}+\mathbf{C} p_{1}+\cdots+\mathbf{C} p_{n}\right) \cup\left(\mathbf{C} q_{1}+\mathbf{C} q_{2}\right)$, so that

$$
\left(\mathfrak{A}_{1},\left.\phi\right|_{\mathfrak{A}_{1}}\right)=\left(\underset{\alpha_{0}}{\mathbf{p}_{0}} \oplus \underset{\alpha_{1}}{\mathbf{C}} \oplus \cdots \oplus \underset{\alpha_{n}}{p_{1}}\right) *\left(\underset{\beta_{1}}{p_{n}} \oplus \underset{\beta_{2}}{\mathbf{C}}\right) .
$$

Let

$$
\begin{aligned}
& L_{+}^{(0)}=\left\{(0, j) \mid \alpha_{0}+\beta_{j}>1\right\}, \\
& L_{0}^{(0)}=\left\{(0, j) \mid \alpha_{0}+\beta_{j}=1\right\} .
\end{aligned}
$$

We use Lemma 4.3 to find that

$$
\mathfrak{A}_{1}=\mathfrak{A}_{1,0}^{r_{1,0}} \oplus \bigoplus_{(i, j) \in L_{+} \cup L_{+}^{(0)}} \stackrel{p_{i} \wedge q_{j}}{\mathbf{C}_{i}+\beta_{j}-1} .
$$

Then by Proposition 2.8, $p_{0} \mathfrak{A}_{1} p_{0}$ and $A_{0}$ freely generate $p_{0} \mathfrak{A} p_{0}$. Hence by Proposition $3.2, p_{0} \mathfrak{A} p_{0}$ is simple. So (40) holds, where $r_{0}=p_{0}+\left(1-p_{0}\right) r_{1,0}$ and where the linear span of

$$
\begin{aligned}
p_{0} \mathfrak{A} p_{0} & +p_{0} \mathfrak{A} p_{0} \mathfrak{A}_{1,0}\left(1-p_{0}\right)+\left(1-p_{0}\right) \mathfrak{A}_{1,0} p_{0} \mathfrak{A} p_{0} \\
& +\left(1-p_{0}\right) \mathfrak{A}_{1,0} p_{0} \mathfrak{A} p_{0} \mathfrak{A}_{1,0}\left(1-p_{0}\right)+\left(1-p_{0}\right) \mathfrak{A}_{1,0}\left(1-p_{0}\right)
\end{aligned}
$$


is dense in $\mathfrak{A}_{0}$. The application of Lemma 4.3 gives for each $(i, j) \in L_{0} \cup L_{0}^{(0)}$ a $*$-homomorphism $\pi_{(i, j)}^{(1)}: \mathfrak{A}_{1,0} \rightarrow \mathbf{C}$ such that $\pi_{(i, j)}^{(1)}\left(r_{1,0} p_{i}\right)=1=\pi_{(i, j)}^{(1)}\left(r_{1,0} q_{j}\right)$, and then $r_{1,0} p_{0}$ is full in

$$
\mathfrak{A}_{1,0} \cap \bigcap_{(i, j) \in L_{0}} \operatorname{ker} \pi_{(i, j)}^{(1)} .
$$

For each $(i, j) \in L_{0}, \pi_{(i, j)}^{(1)}$ extends to a $*$-homomorphism $\pi_{(i, j)}: \mathfrak{A}_{0} \rightarrow \mathbf{C}$ whose kernel is densely spanned by

$$
\begin{aligned}
p_{0} \mathfrak{A} p_{0} & +p_{0} \mathfrak{A} p_{0} \mathfrak{A}_{1,0}\left(1-p_{0}\right)+\left(1-p_{0}\right) \mathfrak{A}_{1,0} p_{0} \mathfrak{A} p_{0} \\
& +\left(1-p_{0}\right) \mathfrak{A}_{1,0} p_{0} \mathfrak{A} p_{0} \mathfrak{A}_{1,0}\left(1-p_{0}\right)+\left(1-p_{0}\right)\left(\operatorname{ker} \pi_{(i, j)}^{(1)}\right)\left(1-p_{0}\right) .
\end{aligned}
$$

Since the algebra (44) contains both $\left(1-p_{0}\right)\left(\mathfrak{A}_{1,0} \cap \bigcap_{(i, j) \in L_{0}} \operatorname{ker} \pi_{(i, j)}^{(1)}\left(1-p_{0}\right)\right)$ and $\left(1-p_{0}\right) \mathfrak{A}_{1,0} p_{0}$, from the denseness of $(44)$ in $\mathfrak{A}_{0}$ we see that $p_{0}$ is full in $\mathfrak{A}_{00}$. Since $p_{0} \mathfrak{A} p_{0}$ is simple, by Proposition 2.6 this implies that $\mathfrak{A}_{00}$ is simple (hence when $L_{0}$ is empty, $\mathfrak{A}_{0}$ is simple).

From the facts, for $(i, j) \in L_{0}$, that $r_{1,0} p_{i}$ is full in

$$
\mathfrak{A}_{1,0} \cap \bigcap_{(0, j) \in L_{0}^{(0)}} \operatorname{ker} \pi_{(0, j)}^{(1)} \cap \bigcap_{\substack{\left(i^{\prime}, j\right) \in L_{0} \\ i^{\prime} \neq i}} \operatorname{ker} \pi_{\left(i^{\prime}, j\right)}^{(1)},
$$

which by a dimension argument contains a nonzero element of $p_{0} \mathfrak{A}_{1,0} p_{0}$, and that every positive element of $p_{0} \mathfrak{A} p_{0}$ is full in $\mathfrak{A}_{00}$, we obtain that $r_{0} p_{i}$ is full in (41), proving (ii).

Because $r_{1,0} q_{j}$ is full in

$$
\mathfrak{A}_{1,0} \cap \bigcap_{\substack{\left(0, j^{\prime}\right) \in L_{0}^{(0)} \\ j^{\prime} \neq j}} \operatorname{ker} \pi_{(0, j)}^{(1)} \cap \bigcap_{\substack{\left(i . j^{\prime}\right) \in L_{0} \\ j^{\prime} \neq j}} \operatorname{ker} \pi_{\left(i, j^{\prime}\right)}^{(1)},
$$

which meets $p_{0} \mathfrak{A}_{1,0} p_{0}$, we similarly obtain that $r_{0} q_{j}$ is full in (41), proving (iii).

If $\left.\phi\right|_{A_{0}}$ is a trace then, by Propositions 3.2 and $3.4, p_{0} \mathfrak{A} p_{0}$ has unique tracial state and stable rank 1 . Since $p_{0} \mathfrak{A} p_{0}$ is full in $\mathfrak{A}_{00}$, by Proposition 2.5 the same hold for $\mathfrak{A}_{00}$ (which is just $\mathfrak{A}_{0}$ when $L_{0}$ is empty), and $\left.\phi\left(r_{0}\right)^{-1} \phi\right|_{\mathfrak{A}_{00}}$ is then seen to be the unique tracial state.

If $\left.\phi\right|_{A_{0}}$ is not a trace, then by Proposition $3.2, p_{0} \mathfrak{A} p_{0}$ has no tracial state, so neither does $\mathfrak{A}_{00}$ have a tracial state.

The following proposition proves Theorem 1.

Proposition 4.5. Let

$$
(\mathfrak{A}, \phi)=\left(\underset{\alpha_{1}}{p_{1}} \oplus \cdots \oplus \underset{\alpha_{n}}{\mathbf{C}}\right) *\left(\underset{\beta_{1}}{q_{1}} \oplus \cdots \oplus \underset{\beta_{m}}{\mathbf{C}}\right),
$$

where $n \geq 2$ and $m \geq 3$.

Then the stable rank of $\mathfrak{A}$ is 1 .

Let

$$
\begin{aligned}
L_{+} & =\left\{(i, j) \mid \alpha_{i}+\beta_{j}>1\right\}, \\
L_{0} & =\left\{(i, j) \mid \alpha_{i}+\beta_{j}=1\right\} .
\end{aligned}
$$


Then

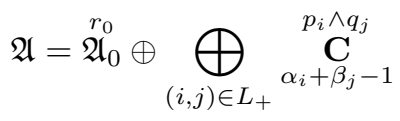

where the centralizer of $\left.\phi\right|_{\mathfrak{A}_{0}}$ has a unital, diffuse abelian subalgebra containing $r_{0} p_{1}$.

If $L_{0}$ is empty then $\mathfrak{A}_{0}$ is simple and nonunital, and $\left.\phi\left(r_{0}\right)^{-1} \phi\right|_{\mathfrak{A}_{0}}$ is the unique tracial state on $\mathfrak{A}_{0}$.

If $L_{0}$ is not empty, then for every $(i, j) \in L_{0}$ there is a $*$-homomorphism $\pi_{(i, j)}$ : $\mathfrak{A}_{0} \rightarrow \mathbf{C}$ such that $\pi_{(i, j)}\left(r_{0} p_{i}\right)=1=\pi_{(i, j)}\left(r_{0} q_{j}\right)$. Then:

(i)

$$
\mathfrak{A}_{00} \stackrel{\text { def }}{=} \bigcap_{(i, j) \in L_{0}} \operatorname{ker} \pi_{(i, j)}
$$

is simple and nonunital, and $\left.\phi\left(r_{0}\right)^{-1} \phi\right|_{\mathfrak{A}_{00}}$ is the unique tracial state on $\mathfrak{A}_{00}$.

(ii) For each $i \in\{1,2, \ldots, n\}, r_{0} p_{i}$ is full in $\mathfrak{A}_{0} \cap \bigcap_{\substack{\left.i^{\prime}, j\right) \in L_{0} \\ i^{\prime} \neq i}} \operatorname{ker} \pi_{\left(i^{\prime}, j\right)}$.

Proof. We proceed by induction on $\min (n, m)$. If $\min (n, m)=2$ then Lemma 4.3 applies to prove the desired results. If $\min (n, m) \geq 3$, take $n \leq m$ and let $\mathfrak{A}_{1}$ be the $\mathrm{C}^{*}$-subalgebra of $\mathfrak{A}$ generated by $\left(\mathbf{C}\left(p_{1}+p_{2}\right)+\mathbf{C} p_{3}+\cdots \mathbf{C} p_{n}\right) \cup\left(\mathbf{C} q_{1}+\cdots+\mathbf{C} q_{m}\right)$. Thus

$$
\left(\mathfrak{A}_{1},\left.\phi\right|_{\mathfrak{A}_{1}}\right) \cong\left(\underset{\alpha_{1}+\alpha_{2}}{\mathrm{p}_{1}+p_{2}} \oplus \underset{\alpha_{3}}{\mathbf{C}} \oplus \cdots \oplus \underset{\alpha_{n}}{\stackrel{p_{3}}{\mathbf{C}}}\right) *\left(\underset{\beta_{1}}{\mathbf{C}} \oplus \cdots \oplus \underset{\beta_{m}}{\mathbf{q _ { 1 }}}\right) .
$$

By the inductive hypothesis, letting $L_{+}, L_{0}, L_{+}^{(1)}, L_{0}^{(1)}, L_{+}^{\prime}, L_{0}^{\prime}, L_{+}^{(2)}$ and $L_{0}^{(2)}$ be as in (35), we have

$$
\mathfrak{A}_{1}=\mathfrak{A}_{1,0}^{r_{1,0}} \oplus \bigoplus_{j \in L_{+}^{\prime}} \underset{\alpha_{1}+\alpha_{2}+\beta_{j}-1}{\stackrel{\left(p_{1}+p_{2}\right) \wedge q_{j}}{\mathbf{C}}} \oplus \bigoplus_{(i, j) \in L_{+}^{(1)}} \underset{\alpha_{i}+\beta_{j}-1}{p_{i} \wedge q_{j}},
$$

and there is a unital, diffuse abelian subalgebra of the centralizer of $\left.\phi\right|_{\mathfrak{A}_{1,0}}$ containing $r_{1,0}\left(p_{1}+p_{2}\right)$. By Proposition 2.8, $\left(p_{1}+p_{2}\right) \mathfrak{A}\left(p_{1}+p_{2}\right)$ is freely generated by $\left(p_{1}+p_{2}\right) \mathfrak{A}_{1}\left(p_{1}+p_{2}\right)$ and $\left(\mathbf{C} p_{1}+\mathbf{C} p_{2}\right)$, so

$$
\begin{aligned}
& \left(p_{1}+p_{2}\right) \mathfrak{A}\left(p_{1}+p_{2}\right)
\end{aligned}
$$

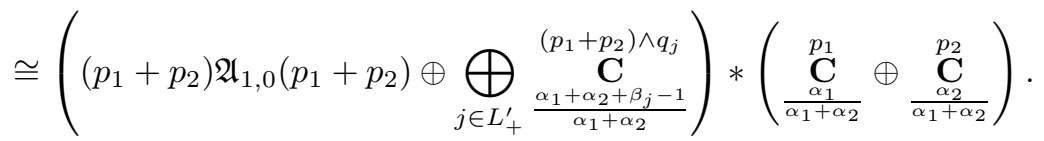

Applying Lemma 4.4 yields

$$
\left(p_{1}+p_{2}\right) \mathfrak{A}\left(p_{1}+p_{2}\right)=\mathfrak{A}_{2,0}^{r_{2,0}} \oplus \underset{(i, j) \in L_{+}^{(2)}}{\bigoplus} \underset{\alpha_{i}+\beta_{j}-1}{\stackrel{p_{i} \wedge q_{j}}{\mathbf{C}}} .
$$

Hence

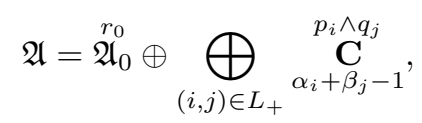

where $r_{0}=r_{2,0}+r_{1,0}\left(1-p_{1}-p_{2}\right)$ and the linear span of the set in (27) is dense in $\mathfrak{A}_{0}$. 
Now the proof of this proposition follows verbatim the proof of Lemma 4.3 after equation (36), except we must also show that when $L_{0}$ is nonempty then $\mathfrak{A}_{00}$ is nonunital. Suppose for contradiction that $\mathfrak{A}_{00}$ is unital with identity $e$. Then $e \neq r_{0}$, but $e x=e x e=x e$ for every $x \in \mathfrak{A}_{0}$. Thus $e$ is in the center of $\mathfrak{A}_{0}$. But by the results of [5], the strong-operator closure of $\mathfrak{A}_{0}$ (in the GNS representation associated to $\left.\left.\phi\right|_{\mathfrak{A}_{0}}\right)$ is a $\mathrm{II}_{1}$-factor. This gives a contradiction, because $e$ must be in the center of this von Neumann algebra.

Remark 4.6. By the same proof, one shows that for every nonempty subset $F$ of $L_{0}$, the ideal $\bigcap_{(i, j) \in F} \operatorname{ker} \pi_{(i, j)}$ of $\mathfrak{A}_{0}$ is nonunital.

The following lemma can be proved from Proposition 4.5 using Proposition 2.8 in the same way that Lemma 4.4 was proved from Lemma 4.3.

Lemma 4.7. Let

$$
(\mathfrak{A}, \phi)=\left(A_{0} \oplus \underset{\alpha_{1}}{\stackrel{p_{1}}{\mathbf{C}}} \oplus \cdots \oplus \underset{\alpha_{n}}{\mathbf{p _ { n }}}\right) *\left(\underset{\beta_{1}}{\mathbf{C}} \oplus \cdots \oplus \underset{\beta_{m}}{\mathbf{q}}\right),
$$

where the centralizer $\left.\phi\right|_{A_{0}}$ has a unital, diffuse abelian subalgebra and where $n \geq 1$, $m \geq 2$. Or, let

$$
(\mathfrak{A}, \phi)=\left(A_{0} \oplus \underset{\alpha_{1}}{p_{1}} \oplus \cdots \oplus \underset{\alpha_{n}}{\mathbf{C}}\right) *\left(B_{0} \oplus \underset{\beta_{1}}{\mathbf{q _ { 1 }}} \oplus \cdots \oplus \underset{\beta_{m}}{\mathbf{C}}\right),
$$

where the centralizer of each of $\left.\phi\right|_{A_{0}}$ and $\left.\phi\right|_{B_{0}}$ has a unital, diffuse abelian subalgebra and where $n \geq 1, m \geq 1$.

Then

$$
\mathfrak{A}=\mathfrak{A}_{0}^{r_{0}} \oplus \bigoplus_{(i, j) \in L_{+}} \stackrel{p_{i} \wedge q_{j}}{\mathbf{C}^{\mathbf{C}}+\beta_{j}-1},
$$

where the centralizer of $\left.\phi\right|_{\mathfrak{A}_{0}}$ has a unital, diffuse abelian subalgebra containing $r_{0} p_{1}$ and another containing $r_{0} q_{1}$.

If $L_{0}$ is empty then $\mathfrak{A}_{0}$ is simple.

If $L_{0}$ is not empty, then for every $(i, j) \in L_{0}$ there is a $*$-homomorphism $\pi_{(i, j)}$ : $\mathfrak{A}_{0} \rightarrow \mathbf{C}$ such that $\pi_{(i, j)}\left(r_{0} p_{i}\right)=1=\pi_{(i, j)}\left(r_{0} q_{j}\right)$. Then:

(i)

$$
\mathfrak{A}_{00} \stackrel{\text { def }}{=} \bigcap_{(i, j) \in L_{0}} \operatorname{ker} \pi_{(i, j)}
$$

is simple and nonunital, and $\left.\phi\left(r_{0}\right)^{-1} \phi\right|_{\mathfrak{A}_{00}}$ is the unique tracial state on $\mathfrak{A}_{00}$.

(ii) For each $i \in\{1,2, \ldots, n\}, r_{0} p_{i}$ is full in $\mathfrak{A}_{0} \cap \bigcap_{\substack{\left(i^{\prime}, j\right) \in L_{0} \\ i^{\prime} \neq i}} \operatorname{ker} \pi_{\left(i^{\prime}, j\right)}$.

(iii) For each $j \in\{1,2, \ldots, m\}, r_{0} p_{j}$ is full in $\mathfrak{A}_{0} \cap \bigcap_{\substack{\left(i, j^{\prime}\right) \in L_{0} \\ j^{\prime} \neq j}} \operatorname{ker} \pi_{\left(i, j^{\prime}\right)}$.

Moreover, if $\phi_{A_{0}}$ is a trace (and if, in the case of (47), $\left.\phi\right|_{B_{0}}$ is a trace), then $\mathfrak{A}$ has stable rank 1 and $\phi\left(r_{0}\right)^{-1} \phi$ is the unique tracial state on $\mathfrak{A}_{0}$ when $L_{0}$ is empty or $\mathfrak{A}_{00}$ when $L_{0}$ is nonempty. Otherwise, $\mathfrak{A}_{0}$, respectively $\mathfrak{A}_{00}$, has no tracial state.

As promised in $\S 1$, a result similar to 4.5 holds for free products of more than two finite dimensional abelian $\mathrm{C}^{*}$-algebras. 
Theorem 4.8. Let $N \in \mathbf{N}, N \geq 3$, and for each $\iota \in\{1, \ldots, N\}$ take a finite dimensional abelian $C^{*}$-algebra and faithful tracial state,

$$
\left(A_{\iota}, \tau_{\iota}\right)=\underset{\alpha_{\iota, 1}}{p_{\iota, 1}} \oplus \underset{\alpha_{\iota, 2}}{p_{\iota, 2}} \oplus \cdots \oplus \underset{\alpha_{\iota, n(\iota)}}{\mathbf{P}},
$$

where $n(\iota) \in \mathbf{N}, n(\iota) \geq 2$. Let

$$
(\mathfrak{A}, \tau)=\underset{\iota=1}{*}\left(A_{\iota}, \tau_{\iota}\right)
$$

and let

$$
\begin{aligned}
& L_{+}=\left\{(j(\iota))_{\iota=1}^{N} \mid \sum_{\iota=1}^{N}\left(1-\alpha_{\iota, j(\iota)}<1\right\},\right. \\
& L_{0}=\left\{(j(\iota))_{\iota=1}^{N} \mid \sum_{\iota=1}^{N}\left(1-\alpha_{\iota, j(\iota)}\right)=1\right\} .
\end{aligned}
$$

Then

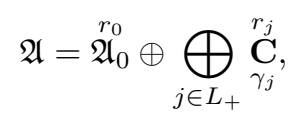

where for $j=(j(\iota))_{\iota=1}^{N} \in L_{+}, \gamma_{j}=1-\sum_{\iota=1}^{N}\left(1-\alpha_{\iota, j(\iota)}\right)$ and $r_{j}=\bigwedge_{\iota=1}^{N} p_{\iota, j(\iota)}$. For each $1 \leq \iota \leq N$ and each $1 \leq k \leq n(\iota), \mathfrak{A}_{0}$ has a unital, diffuse abelian subalgebra which contains $r_{0} p_{\iota, k}$. Moreover, $\mathfrak{A}$ has stable rank 1.

If $L_{0}$ is empty, then $\mathfrak{A}_{0}$ is simple and $\left.\phi\left(r_{0}\right)^{-1} \phi\right|_{\mathfrak{A}_{0}}$ is the unique tracial state on $\mathfrak{A}_{0}$.

If $L_{0}$ is nonempty, then for every $j=(j(\iota))_{\iota=1}^{N} \in L_{0}$ there is a $*$-homomorphism $\pi_{j}: \mathfrak{A}_{0} \rightarrow \mathbf{C}$ such that $\forall 1 \leq \iota \leq N \pi_{j}\left(p_{\iota, j(\iota)}\right)=1$. Then:

(i)

$$
\mathfrak{A}_{00} \stackrel{\text { def }}{=} \bigcap_{j \in L_{0}} \operatorname{ker} \pi_{j}
$$

is simple and nonunital, and $\left.\phi\left(r_{0}\right)^{-1} \phi\right|_{\mathfrak{A}_{00}}$ is the unique tracial state on $\mathfrak{A}_{00}$.

(ii) For each $1 \leq \iota \leq N$ and each $1 \leq k \leq n(\iota), r_{0} p_{\iota, k}$ is full in

$$
\mathfrak{A}_{0} \cap \bigcap_{\substack{j \in L_{0} \\ j(\iota) \neq k}} \operatorname{ker} \pi_{j} .
$$

Proof. The proof is by induction on $N$, and the case $N=3$ and the inductive step are proved simultaneously. Let $\mathfrak{A}_{N-1}$ be the $\mathrm{C}^{*}$-subalgebra of $\mathfrak{A}$ generated by $\bigcup_{\iota=1}^{N-1} A_{\iota}$. Use the inductive hypothesis (or, when $N=3$, Proposition 4.5 or Proposition 2.7) to find that

$$
\left(\mathfrak{A}_{N-1},\left.\tau\right|_{\mathfrak{A}_{N-1}}\right) \cong \underset{\iota=1}{*} \stackrel{N}{*}^{*}\left(A_{\iota}, \tau_{\iota}\right)
$$

Then

$$
(\mathfrak{A}, \tau) \cong\left(\mathfrak{A}_{N-1},\left.\tau\right|_{\mathfrak{A}_{N-1}}\right) *\left(A_{N}, \tau_{N}\right),
$$

and one uses Lemma 4.7 to find $\mathfrak{A}$. 
One can generalize this to the free product of finitely many (say $N$ ) algebras of the form $A_{0} \oplus \mathbf{C} \oplus \cdots \oplus \mathbf{C}$, either, as Theorem 4.8 was proved, by using Lemma 4.7 and induction on $N$, or, as Lemma 4.4 was proved, by applying Theorem 4.8 and Lemma 3.2. One obtains the following result.

Theorem 4.9. Let $N \in \mathbf{N}, N \geq 3$, and for each $\iota \in\{1, \ldots, N\}$ let $\left(A_{\iota}, \phi_{\iota}\right)$ be either a finite dimensional abelian algebra with a faithful state as in (48) or

$$
\left(A_{\iota}, \phi_{\iota}\right)=A_{\iota, 0} \oplus \underset{\alpha_{\iota, 1}}{p_{\iota, 1}} \oplus \underset{\alpha_{\iota, 2}}{p_{\iota, 2}} \oplus \cdots \oplus \underset{\alpha_{\iota, n(\iota)}}{\mathbf{C}},
$$

where $n(\iota) \in \mathbf{N}, n(\iota) \geq 1$, and where the centralizer of $\left.\phi_{\iota}\right|_{A_{\iota, 0}}$ has a unital, diffuse abelian subalgebra. Let

$$
(\mathfrak{A}, \tau)=\underset{\iota=1}{*}\left(A_{\iota}, \tau_{\iota}\right)
$$

and let $L_{+}$and $L_{0}$ be as in (49). Then

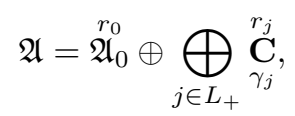

where for $j=(j(\iota))_{\iota=1}^{N} \in L_{+}, \gamma_{j}=1-\sum_{\iota=1}^{N}\left(1-\alpha_{\iota, j(\iota)}\right)$ and $r_{j}=\bigwedge_{\iota=1}^{N} p_{\iota, j(\iota)}$. If each $\left.\phi_{\iota}\right|_{A_{\iota, 0}}$ is a trace then $\mathfrak{A}$ has stable rank 1 .

If $L_{0}$ is empty then $\mathfrak{A}_{0}$ is simple, and if each $\left.\phi_{\iota}\right|_{A_{\iota, 0}}$ is a trace then $\left.\phi\left(r_{0}\right)^{-1} \phi\right|_{\mathfrak{A}_{0}}$ is the unique tracial state on $\mathfrak{A}_{0}$. If some $\left.\phi_{\iota}\right|_{A_{\iota, 0}}$ is not a trace then $\mathfrak{A}_{0}$ has no tracial states.

If $L_{0}$ is nonempty, then for every $j=(j(\iota))_{\iota=1}^{N} \in L_{0}$ there is a *-homomorphism $\pi_{j}: \mathfrak{A}_{0} \rightarrow \mathbf{C}$ such that $\forall 1 \leq \iota \leq N \pi_{j}\left(p_{\iota, j(\iota)}\right)=1$. Then

$$
\mathfrak{A}_{00} \stackrel{\text { def }}{=} \bigcap_{j \in L_{0}} \operatorname{ker} \pi_{j}
$$

is simple and nonunital, and if each $\left.\phi_{\iota}\right|_{A_{\iota, 0}}$ is a trace then $\left.\phi\left(r_{0}\right)^{-1} \phi\right|_{\mathfrak{A}_{00}}$ is the unique tracial state on $\mathfrak{A}_{00}$. If some $\left.\phi_{\iota}\right|_{A_{\iota, 0}}$ is not a trace then $\mathfrak{A}_{00}$ has no tracial states.

Restricting the above proposition to the case when each $A_{\iota, 0}$ is abelian, we obtain the following result about the free product of finitely many abelian $\mathrm{C}^{*}$-algebras.

Corollary 4.10. Let $N \in \mathbf{N}, N \geq 2$, and for each $1 \leq \iota \leq N$ consider the abelian $C^{*}$-algebra and state $\left(C\left(X_{\iota}\right), \int \cdot d \mu_{\iota}\right)$, where $\mu_{\iota}$ is a regular Borel probability measure on $X_{\iota}$ whose support is all of $X_{\iota}$ and having at most finitely many atoms, each of which is an isolated point of $X_{\iota}$. Let

$$
(\mathfrak{A}, \tau)=\underset{\iota=1}{*}\left(C\left(X_{\iota}\right), \int \cdot d \mu_{\iota}\right) .
$$

Let

$$
\begin{aligned}
& L_{+}=\left\{x=\left(x_{\iota}\right)_{\iota=1}^{N} \mid x_{\iota} \in X_{\iota}, \sum_{\iota=1}^{N}\left(1-\mu_{\iota}\left(\left\{x_{\iota}\right\}\right)\right)<1\right\}, \\
& L_{0}=\left\{x=\left(x_{\iota}\right)_{\iota=1}^{N} \mid x_{\iota} \in X_{\iota}, \sum_{\iota=1}^{N}\left(1-\mu_{\iota}\left(\left\{x_{\iota}\right\}\right)\right)=1\right\} .
\end{aligned}
$$


Assume that no $X_{\iota}$ is a one-point space, and also exclude the case when $N=2$ and $X_{1}$ and $X_{2}$ are both two-point spaces. Then $\mathfrak{A}$ has stable rank 1 , and

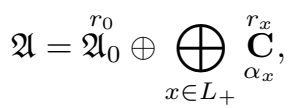

where $\alpha_{x}=1-\sum_{\iota=1}^{N}\left(1-\mu_{\iota}\left(\left\{x_{\iota}\right\}\right)\right)$. If $L_{0}$ is empty, then $\mathfrak{A}_{0}$ is simple and has unique tracial state $\left.\tau\left(r_{0}\right)^{-1} \tau\right|_{\mathfrak{A}_{0}}$.

If $L_{0}$ is nonempty, then there are distinct, surjective $*$-homomorphisms, $\pi_{x}$ : $\mathfrak{A}_{0} \rightarrow \mathbf{C},\left(x \in L_{0}\right)$, such that

$$
\mathfrak{A}_{00} \stackrel{\text { def }}{=} \bigcap_{x \in L_{0}} \operatorname{ker} \pi_{x}
$$

is simple and nonunital and has unique tracial state $\left.\tau\left(r_{0}\right)^{-1} \tau\right|_{\mathfrak{A}_{00}}$.

\section{More general abelian $\mathrm{C}^{*}$-Algebras}

In this section we will investigate free products,

$$
(\mathfrak{A}, \tau)=\underset{\iota=1}{*} \underset{*}{*}\left(C\left(X_{\iota}\right), \int \cdot d \mu_{\iota}\right),
$$

of abelian $\mathrm{C}^{*}$-algebras and states, $\left(C\left(X_{\iota}\right), \int \cdot d \mu_{\iota}\right)$, each of which can be written as an inductive limit of the abelian algebras and states considered in Corollary 4.10. The criterion for simplicity of the free product of such abelian algebras is the same as for finite dimensional abelian algebras, namely, $\mathfrak{A}$ is simple if and only if there are no atoms $x_{\iota} \in X_{\iota}$ of $\mu_{\iota}(1 \leq \iota \leq N)$ such that $\sum_{1}^{N}\left(1-\mu\left(\left\{x_{\iota}\right\}\right)\right) \leq 1$. (Compare Corollary 4.10.) However, in the case when there are atoms $x_{\iota}$ satisfying $\sum_{1}^{N}\left(1-\mu\left(\left\{x_{\iota}\right\}\right)\right)<1$, we don't always get a corresponding copy of $\mathbf{C}$ as a direct summand of $\mathfrak{A}$. In fact we get such a direct summand, i.e. a minimal and central projection in $\mathfrak{A}$, like $r_{x}$ in (51), corresponding to these atoms, if and only if each $x_{\iota}$ is an isolated point of $X_{\iota}$.

Definition 5.1. Let $X$ be a compact Hausdorff topological space and let $\mu$ be a regular Borel probability measure on $X$. We say $(X, \mu)$ is an inverse limit of spaces and measures with isolated atoms if $X$ is an inverse limit, $X=\lim \left(X_{n}, \kappa_{n}\right)$, of compact Hausdorff spaces $X_{n}$ and surjective, continuous maps $\kappa_{n}: X_{n+1} \rightarrow X_{n}$, $(n \in \mathbf{N})$, such that, letting $\lambda_{n}: X \rightarrow X_{n}$ be the resulting canonical surjective maps and letting $\mu_{n}=\left(\lambda_{n}\right)_{*}(\mu)$ be the push-forward measures, each $\mu_{n}$ has at most finitely many atoms and each atom of $\mu_{n}$ is an isolated point of $X_{n}$, and, moreover, if $x \in X_{n}$ and if $\kappa_{n}^{-1}(\{x\})$ has more than one point, then $x$ is an atom of $\mu_{n}$.

Examples 5.2. In each of the following cases, $(X, \mu)$ is an inverse limit of spaces and measures with isolated atoms. (One can easily cook up more intricate examples as well.)

(i) $\mu$ has no atoms;

(ii) all the atoms of $\mu$ are isolated points of $X$;

(iii) $X$ is separable and totally disconnected;

(iv) $X=\{0\} \cup \bigcup_{n=1}^{\infty}\left[\frac{1}{2 n+1}, \frac{1}{2 n}\right]$ with the relative topology from $\mathbf{R}$, and $\mu$ has a unique atom at 0 . 
Theorem 5.3. Let $N \in \mathbf{N}, N \geq 2$, and for each $1 \leq \iota \leq N$ let $\left(X_{\iota}, \mu_{\iota}\right)$ be a compact Hausdorff space and a regular Borel probability measure, each of which is an inverse limit of spaces and measures with isolated atoms. Assume each $X_{\iota}$ has more than one point and each $\mu_{\iota}$ has support equal to all of $X_{\iota}$. Exclude the case when $N=2$ and $X_{1}$ and $X_{2}$ are both two-point spaces. Let

$$
(\mathfrak{A}, \tau)=\underset{\iota=1}{*}\left(C\left(X_{\iota}\right), \int \cdot d \mu_{\iota}\right) .
$$

Let

$$
\begin{aligned}
I_{+} & =\left\{x=\left(x_{\iota}\right)_{\iota=1}^{N} \in \prod_{\iota=1}^{N} X_{\iota} \mid \sum_{\iota=1}^{N}\left(1-\mu_{\iota}\left(\left\{x_{\iota}\right\}\right)\right)<1, \text { each } x_{\iota} \text { is isolated in } X_{\iota}\right\} \\
L & =\left\{x=\left(x_{\iota}\right)_{\iota=1}^{N} \in \prod_{\iota=1}^{N} X_{\iota} \mid \sum_{\iota=1}^{N}\left(1-\mu_{\iota}\left(\left\{x_{\iota}\right\}\right)\right) \leq 1\right\} \backslash I_{+} .
\end{aligned}
$$

Then $\mathfrak{A}$ has stable rank 1 , and

$$
\mathfrak{A}=\stackrel{r_{0}}{\mathfrak{A}} 0 \bigoplus_{x \in I_{+}} \underset{\alpha_{x}}{\stackrel{r_{x}}{\mathbf{C}}}
$$

where $\alpha_{x}=1-\sum_{\iota=1}^{N}\left(1-\mu_{\iota}\left(\left\{x_{\iota}\right\}\right)\right)$ and where $r_{x}=\bigwedge_{\iota=1}^{N} p_{x_{\iota}}$, with $p_{x_{\iota}} \in C\left(X_{\iota}\right)$ the characteristic function of $\left\{x_{\iota}\right\}$. If $L$ is empty, then $\mathfrak{A}_{0}$ is simple and has unique tracial state $\left.\tau\left(r_{0}\right)^{-1} \tau\right|_{\mathfrak{A}_{0}}$.

If $L$ is nonempty, then for every $x \in L$ there is a $*$-homomorphism $\pi_{x}: \mathfrak{A}_{0} \rightarrow \mathbf{C}$ such that whenever $f \in C\left(X_{\iota}\right)$ we have $\pi_{x}(f)=f\left(x_{\iota}\right)$. Moreover,

$$
\mathfrak{A}_{00} \stackrel{\text { def }}{=} \bigcap_{x \in L} \operatorname{ker} \pi_{x}
$$

is simple and nonunital, and has unique tracial state. Finally, for each nonempty subset $F \subseteq L$, the ideal $\bigcap_{x \in F} \operatorname{ker} \pi_{x}$ of $\mathfrak{A}_{0}$ is nonunital.

Proof. Let $X_{\iota}=\varliminf_{\llcorner}\left(X_{\iota, n}, \kappa_{\iota, n}\right)$ be an inverse limit with properties as in Definition 5.1, and let $\lambda_{\iota, n}: X_{\iota} \rightarrow X_{\iota, n}$ and $\mu_{\iota, n}=\left(\lambda_{\iota, n}\right)_{*}\left(\mu_{\iota}\right)$ be the corresponding map and measure. Thus, we may regard $C\left(X_{\iota, n}\right)$ as a unital $\mathrm{C}^{*}$-subalgebra of $C\left(X_{\iota}\right)$, where the state $\int \cdot d \mu_{\iota}$ restricts to $\int \cdot d \mu_{\iota, n}$, and $C(X)=\overline{\bigcup_{n=1}^{\infty} C\left(X_{\iota, n}\right)}$. If $y \in X_{\iota, n}$ is an atom of $\mu_{\iota, n}$ and if $\lambda_{\iota, n}^{-1}(\{y\})$ contains no atoms of $\mu_{\iota}$, then since $\lambda_{\iota, n}^{-1}(\{y\})$ is clopen in $X_{\iota}$ we may change $\left(X_{\iota, n}, \mu_{\iota, n}\right)$ by substituting $\lambda_{\iota, n}^{-1}(\{y\})$ for $y$ and changing $\mu_{\iota, n}$ accordingly. Then we must modify every $\left(X_{\iota, n+k}, \mu_{\iota, n+k}\right)$ too. By doing so, we may assume without loss of generality that whenever $n \in \mathbf{N}$ and $y \in X_{\iota, n}$ is an atom of $\mu_{\iota, n}$, then $\lambda_{\iota, n}^{-1}(\{y\})$ contains an atom of $\mu_{\iota}$.

Let $c=\max \left\{\mu_{\iota}(\{x\}) \mid x \in X_{\iota}, 1 \leq \iota \leq N\right\}$. Then $c<1$. If $x_{\iota} \in X_{\iota}$ appears as one of the coordinates in an element of $L \cup I_{+}$, then $1-\mu_{\iota}\left(\left\{x_{\iota}\right\}\right)+(N-1)(1-c) \leq 1$, so $\mu_{\iota}\left(\left\{x_{\iota}\right\}\right) \geq(N-1)(1-c)$. Therefore, $L \cup I_{+}$is finite. Moreover, if $\mu_{\iota}$ has infinitely many atoms then their masses form a sequence tending to zero, so there is $\epsilon>0$ such that whenever $x_{\iota} \in X_{\iota}(1 \leq \iota \leq N)$ and $\left(x_{\iota}\right)_{\iota=1}^{N} \notin L \cup I_{+}$then $\sum_{\iota=1}^{N}\left(1-\mu\left(\left\{x_{\iota}\right\}\right)\right)>1+\epsilon$.

Let $x_{\iota} \in X_{\iota}$ be an atom of $\mu_{\iota}$. If $x_{\iota}$ is an isolated point of $X_{\iota}$, then for $n$ large enough $\lambda_{\iota, n}^{-1}\left(\left\{\lambda_{\iota, n}\left(x_{\iota}\right)\right\}\right)=\left\{x_{\iota}\right\}$ and hence the characteristic function of $\left\{x_{\iota}\right\}$, which we called $p_{x_{\iota}}$, is in $C\left(X_{\iota, n}\right)$. We assume without loss of generality that this holds for every $n$ and for every $\iota$. If $x_{\iota}$ is not an isolated point of $X_{\iota}$ then $\left(\lambda_{\iota, n}^{-1}\left(\left\{\lambda_{\iota, n}\left(x_{\iota}\right)\right\}\right)\right)_{n=1}^{\infty}$ 
is a neighborhood base for $x_{\iota}$ and, since $\mu_{\iota}$ is a regular measure whose support is all of $X_{\iota}$, we have

$$
\mu_{\iota, n}\left(\left\{\lambda_{\iota, n}\left(x_{\iota}\right)\right\}\right) \geq \mu_{\iota, n+1}\left(\left\{\lambda_{\iota, n+1}\left(x_{\iota}\right)\right\}\right)>\mu_{\iota}\left(\left\{x_{\iota}\right\}\right)
$$

and $\lim _{n \rightarrow \infty} \mu_{\iota, n}\left(\left\{\lambda_{\iota, n}\left(x_{\iota}\right)\right\}\right)=\mu_{\iota}\left(\left\{x_{\iota}\right\}\right)$. Thus, for $n$ large enough we have, for every atom, $x_{\iota} \in X_{\iota}$ of $\mu_{\iota}$,

$$
\mu_{\iota, n}\left(\left\{\lambda_{\iota, n}\left(x_{\iota}\right)\right\}\right)<\mu_{\iota}\left(\left\{x_{\iota}\right\}\right)+\epsilon / N
$$

We assume without loss of generality this holds for every $n$ and for every $\iota$.

Then, whenever $x_{\iota, n} \in X_{\iota, n}$ is an atom of $\mu_{\iota, n}$ and

$$
\sum_{\iota=1}^{N}\left(1-\mu_{\iota, n}\left(x_{\iota, n}\right)\right) \leq 1,
$$

there is a unique $\left(x_{\iota}\right)_{\iota=1}^{N} \in L \cup I_{+}$such that $x_{\iota, n}=\lambda_{\iota, n}\left(x_{\iota}\right)(1 \leq \iota \leq N)$. Moreover, if $\left(x_{\iota}\right)_{\iota=1}^{N} \in I_{+}$then the atoms $\lambda_{\iota, n}\left(x_{\iota}\right)$ and $x_{\iota}$ have the same mass, and if equality holds in (53) then each $x_{\iota}$ is an isolated point of $X_{\iota}$.

Let $\mathfrak{A}_{n}$ be the $\mathrm{C}^{*}$-subalgebra of $\mathfrak{A}$ generated by $\bigcup_{\iota=1}^{N} C\left(X_{\iota, n}\right)$. Then

$$
\mathfrak{A}_{n} \cong \underset{\iota=1}{*} \boldsymbol{*}_{(=1}\left(C\left(X_{\iota, n}\right), \int \cdot d \mu_{\iota, n}\right),
$$

and by Corollary 4.10 and the facts discussed above we have

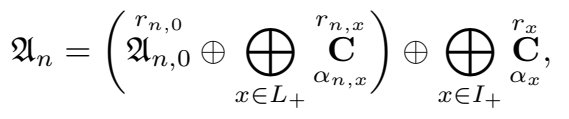

where $I_{+}, \alpha_{x}$ and $r_{x}$ are as in the statement of the proposition and where

$$
\begin{aligned}
L_{+} & =\left\{x=\left(x_{\iota}\right)_{\iota=1}^{N} \in L \mid \sum_{\iota=1}^{N}\left(1-\mu_{\iota}\left(\left\{x_{\iota}\right\}\right)\right)<1\right\}, \\
\alpha_{n, x} & =1-\sum_{\iota=1}^{N}\left(1-\mu_{\iota, n}\left(\left\{\lambda_{\iota, n}\left(x_{\iota}\right)\right\}\right)\right), \\
r_{n, x} & =\bigwedge_{\iota=1}^{N} p_{x_{\iota}, n},
\end{aligned}
$$

where $p_{x_{\iota}, n} \in C\left(X_{\iota, n}\right)$ is the characteristic function of $\left\{\lambda_{\iota, n}\left(x_{\iota}\right)\right\}$. Also, letting $L_{0}=L \backslash L_{+}$, for each $x \in L_{0}$ there is a $*$-homomorphism $\pi_{n, x}: \mathfrak{A}_{n, 0} \rightarrow \mathbf{C}$ sending $p_{x_{\iota}, n}=p_{x_{\iota}}$ to $1(1 \leq \iota \leq N)$.

We have $\mathfrak{A}_{n} \subseteq \mathfrak{A}_{n+1}$ and $\mathfrak{A}=\overline{\bigcup_{n=1}^{\infty} \mathfrak{A}_{n}}$. Let

$$
\mathfrak{A}_{n, 0}^{\prime}=\mathfrak{A}_{n, 0}^{r_{n, 0}} \oplus \bigoplus_{x \in L_{+}} \underset{\alpha_{n, x}}{r_{n, x}}
$$

Then $\mathfrak{A}_{n, 0}^{\prime} \subseteq \mathfrak{A}_{n+1,0}^{\prime}$, and (52) holds with $\mathfrak{A}_{0}=\overline{\bigcup_{n=1}^{\infty} \mathfrak{A}_{n, 0}^{\prime}}$. If $L=\emptyset$ then each $\mathfrak{A}_{n, 0}^{\prime}=\mathfrak{A}_{n, 0}$ is simple with unique tracial state, and thus their inductive limit $\mathfrak{A}_{0}$ is simple with unique tracial state. Suppose $L$ is nonempty. For each $x=\left(x_{\iota}\right)_{\iota=1}^{N} \in L$ let $\pi_{x, n}: \mathfrak{A}_{n, 0}^{\prime} \rightarrow \mathbf{C}$ be the $*$-homomorphism sending $p_{x_{\iota}, n} \mapsto 1(1 \leq \iota \leq N)$. Then

$$
\mathfrak{A}_{n, 00} \stackrel{\text { def }}{=} \bigcap_{x \in L} \operatorname{ker} \pi_{x, n}
$$


is simple with unique tracial state. Clearly $\pi_{x, n+1}$ is an extension of $\pi_{x, n}$, so taking the inductive limit we get, for each $x \in L$, a $*$-homomorphism $\pi_{x}: \mathfrak{A}_{0} \rightarrow \mathbf{C}$. When restricted to $C\left(X_{\iota}\right), \pi_{x}$ gives evaluation at $x_{\iota} \in X_{\iota}$. Then $\mathfrak{A}_{00}$ is the inductive limit of the algebras $\mathfrak{A}_{n, 00}$, and thus is simple with unique tracial state.

We now show that $\mathfrak{A}_{00}$ is nonunital. First consider the case when $L_{+}$is nonempty. Then for each $x=\left(x_{\iota}\right)_{\iota=1}^{N} \in L_{+}, \alpha_{n, x}$ decreases to the limit $\sum_{\iota=1}^{N}\left(1-\mu_{\iota}\left(\left\{x_{\iota}\right\}\right)\right)$ but is never equal to this quantity. Suppose for contradiction that $e \in \mathfrak{A}_{00}$ is the identity of $\mathfrak{A}_{00}$. Let $n \in \mathbf{N}$ and $a \in \mathfrak{A}_{n, 00}$. Then there is $m>n$ such that $\alpha_{n, x}>$ $\alpha_{m, x}$, and thus $r_{n, x}-r_{m, x} \in \mathfrak{A}_{m, 00}$ is a nonzero projection. Since $a \in \operatorname{ker} \pi_{n, x}$, since $\pi_{n, x}\left(r_{n, x}\right)=1$ and since $r_{n, x}$ is a minimal projection of $\mathfrak{A}_{n, 00}$, we must have $a r_{n, x}=0$, and hence $a\left(r_{n, x}-r_{m, x}\right)=0$. But $e\left(r_{n, x}-r_{m, x}\right)=r_{n, x}-r_{m, x}$, so

$$
1=\left\|r_{n, x}-r_{m, x}\right\|=\left\|(e-a)\left(r_{n, x}-r_{m, x}\right)\right\| \leq\|e-a\| .
$$

This contradicts the fact that $e \in \overline{\bigcup_{n} \mathfrak{A}_{n, 00}}$.

If $L_{+}=\emptyset$ then $L_{0}$ is nonempty, so each $\mathfrak{A}_{n, 00}$ is nonunital. But this implies that their inductive limit, $\mathfrak{A}_{00}$, is nonunital.

The same technique shows that each ideal $\bigcap_{x \in F} \operatorname{ker} \pi_{x}$ of $\mathfrak{A}_{0}$ is nonunital.

Although the above proposition was stated only for free products of abelian $\mathrm{C}^{*}$ algebras, a similar result is easily proved for free products of inductive limits of the algebras of the form $A_{0} \oplus \mathbf{C} \oplus \cdots \oplus \mathbf{C}$ that were considered, for example, in Theorem 4.9 .

\section{Free PRODUCTS OF INFINITELy MANY ALGEBRAS}

In this section we consider the reduced free product of infinitely many finite dimensional abelian $\mathrm{C}^{*}$-algebras. Although such free products of infinitely many algebras can fail to be simple, they never get a copy of $\mathbf{C}$ as a direct summand, and hence their proper, nontrivial ideals, if any, are always nonunital. Moreover, the center of the free product algebra is always trivial, even when its von Neumann algebra closure (i.e. the strong-operator closure of the GNS representation) has nontrivial projections that are both minimal and central.

Theorem 6.1. For each $\iota \in \mathbf{N}$ let $\left(A_{\iota}, \tau_{\iota}\right)$ be a finite dimensional abelian algebra with faithful state as in (48). Let

$$
(\mathfrak{A}, \tau)=\underset{\iota=1}{*}\left(A_{\iota}, \tau_{\iota}\right) .
$$

Then $\mathfrak{A}$ has stable rank 1. Let

$$
L=\left\{(j(\iota))_{\iota=1}^{\infty} \mid \sum_{\iota=1}^{\infty}\left(1-\alpha_{\iota, j(\iota)}\right) \leq 1\right\} .
$$

If $L$ is empty then $\mathfrak{A}$ is simple and $\tau$ is the unique tracial state on $\mathfrak{A}$.

Otherwise, if $L$ is nonempty, then for each $j=(j(\iota))_{\iota=1}^{\infty} \in L$ there is a*homomorphism $\pi_{j}: \mathfrak{A} \rightarrow \mathbf{C}$ such that $\pi_{j}\left(p_{\iota, j(\iota)}\right)=1$ for every $\iota \in \mathbf{N}$. Then

$$
\mathfrak{A}_{00} \stackrel{\text { def }}{=} \bigcap_{j \in L} \operatorname{ker} \pi_{j}
$$


is simple and nonunital. Moreover, letting

$$
\gamma_{0}=1-\sum_{j \in L}\left(\sum_{\iota=1}^{\infty}(1-\alpha(\iota, j(\iota)))\right),
$$

$\left.\gamma_{0}^{-1} \tau\right|_{\mathfrak{A}_{00}}$ is the unique tracial state on $\mathfrak{A}_{00}$. Finally, for every nonempty subset $F \subseteq L$, the ideal $\bigcap_{j \in F} \operatorname{ker} \pi_{j}$ is nonunital.

Proof. The proof follows in a straightforward manner by using Theorem 4.8 and taking inductive limits. To show that $\mathfrak{A}_{00}$ and each of the other proper, nontrivial ideals of $\mathfrak{A}$ are nonunital, an argument like that in the proof Theorem 5.3 (in the case $L_{+}$nonempty) is used.

Of course, similar results for free products of infinitely many abelian algebras of the form considered in $\S 5$ or infinitely many algebras of the form $A_{0} \oplus \mathbf{C} \oplus \cdots \oplus \mathbf{C}$ considered in Theorem 4.9 are easily obtained.

\section{Conjectures}

This section contains a couple of related open problems which seem likely to have solutions, though I don't yet see how to find them.

Conjecture 7.1. Let $C(X)$ and $C(Y)$ be unital, abelian $C^{*}$-algebras having faithful states given by probability measures $\mu_{X}$ on $X$ and $\mu_{Y}$ on $Y$. Let

$$
(\mathfrak{A}, \tau)=\left(C(X), \int \cdot d \mu_{X}\right) *\left(C(Y), \int \cdot d \mu_{Y}\right) .
$$

Then a necessary and sufficient condition for $\mathfrak{A}$ to be simple is that for every $x \in X$ and $y \in Y, \mu_{X}(\{x\})+\mu_{Y}(\{y\})<1$.

Proposition 7.2. The conditions in Conjecture 7.1 are necesary for simplicity of $\mathfrak{A}$.

Proof. Suppose the conditions of 7.1 are not satisfied. Let $C \subseteq X$ be the set of atoms of $\mu_{X}$. If $C$ is finite then let $D=C$. If $C$ is infinite then let $\bar{C}$ denote the closure of $C$ in $X$ and let $D$ be a totally disconnected, separable, compact Hausdorff space equipped with a continuous surjective map $\lambda: D \rightarrow \bar{C}$. (That such a space exists is a well-known result.) For each $c \in C$, choose $f(c) \in \lambda^{-1}(D)$. Let $\nu_{D}$ be the measure on $D$ given by $\nu_{D}(\{f(c)\})=\mu_{X}(\{c\})$ and $\nu_{D}(D \backslash f(C))=0$. Replace $D$ by the support of $\nu_{D}$. Let $\nu_{X^{\prime}}$ be the measure on $X$ that when restricted to $X \backslash C$ gives the same measure as $\mu_{X}$ and such that $\nu_{X^{\prime}}(C)=0$, and let $X^{\prime}$ be the support of $\nu_{X^{\prime}}$. Let $X_{a}$ be the compact Hausdorff space that is the disjoint union of $X^{\prime}$ and $D$, and let $\mu_{X_{a}}$ be the measure on $X_{a}$ obtained from $\nu_{X^{\prime}}$ and $\nu_{D}$. Let $\kappa_{X}: X_{a} \rightarrow X$ be the surjective, continous map composed of the inclusion $X^{\prime} \hookrightarrow X$ and $\lambda: D \rightarrow X$. Then $\mu_{X_{a}}$ is the push-forward measure, $\mu_{X_{a}}=\left(\kappa_{X}\right)_{*}\left(\mu_{X}\right)$. Therefore $\kappa_{X}$ induces an injective, unital $*$-homomorphism, $\pi_{X}: C(X) \rightarrow C\left(X_{a}\right)$, preserving the states defined by the measures $\mu_{X_{a}}$ and $\mu_{X}$. Do the same for $Y$, getting $\pi_{Y}: C(Y) \rightarrow C\left(Y_{A}\right)$.

Now Theorem 5.3 applies to the free product

$$
\left(\mathfrak{A}^{\prime}, \tau^{\prime}\right)=\left(C\left(X_{a}\right), \int \cdot d \mu_{X_{a}}\right) *\left(C\left(Y_{a}\right), \int \cdot d \mu_{Y_{a}}\right)
$$

If the condition of Conjecture 7.1 is not satisfied, then there are $x \in X_{a}$ and $y \in Y_{a}$ such that $\mu_{X_{a}}(\{x\})+\mu_{Y_{a}}(\{y\}) \geq 1$, which by 5.3 implies the existence of a $*-$ homomorphism $\pi: \mathfrak{A}^{\prime} \rightarrow \mathbf{C}$ that when restricted to $C\left(X_{a}\right)$ is evaluation at $x$. 
Since $\tau^{\prime}$ is faithful and $\kappa_{X}$ and $\kappa_{Y}$ are trace-preserving inclusions, they induce an inclusion $\mathfrak{A} \hookrightarrow \mathfrak{A}^{\prime}$. Then $\left.\pi\right|_{\mathfrak{A}}$ is a nonzero $*$-homomorphism, so $\mathfrak{A}$ is not simple.

We now look at free products of finite dimensional algebras, and we use the notation of [6] for a faithful state on a finite dimensional algebra. Thus, if $D=$ $\bigoplus_{j=1}^{K} M_{n_{j}}(\mathbf{C})$, we write

$$
(D, \phi)=\bigoplus_{j=1}^{K} M_{n_{j}}(\mathbf{C})
$$

to mean that the restriction of $\phi$ to the $j$ th summand of $D$ is given by $\operatorname{Tr}(\cdot H)$, where $\mathrm{Tr}$ is the unnormalized trace on $M_{n_{j}}(\mathbf{C})$ and where $H$ is the diagonal matrix with $\alpha_{j, 1}, \ldots, \alpha_{j, n_{j}}$ down the diagonal.

Conjecture 7.3. Let

$$
\begin{aligned}
\left(A_{1}, \phi_{1}\right) & =\bigoplus_{j=1}^{K_{1}} M_{\alpha_{j, 1}, \cdots, \alpha_{j, n_{j}}}(\mathbf{C}), \\
\left(A_{2}, \phi_{2}\right) & =\bigoplus_{j=1}^{K_{2}} M_{m_{j, 1}, \cdots, \beta_{j, m_{j}}}(\mathbf{C})
\end{aligned}
$$

be finite dimensional $C^{*}$-algebras with faithful states and let

$$
(\mathfrak{A}, \phi)=\left(A_{1}, \phi_{1}\right) *\left(A_{2}, \phi_{2}\right)
$$

be the reduced free product $C^{*}$-algebra. Then necessary and sufficient conditions for $\mathfrak{A}$ to be simple are that if $n_{j}=1$ for some $j$ then for every $1 \leq k \leq K_{2}$

$$
\frac{1}{1-\alpha_{j, 1}}<\sum_{i=1}^{m_{k}} \frac{1}{\beta_{k, i}}
$$

and if $m_{j}=1$ for some $j$ then for every $1 \leq k \leq K_{1}$

$$
\frac{1}{1-\beta_{j, 1}}<\sum_{i=1}^{n_{k}} \frac{1}{\alpha_{k, i}}
$$

If the above conjecture is true, then in particular $\mathfrak{A}$ is simple whenever each $n_{j}>1$ and each $m_{j}>1$. This conjecture is inspired by the results of [5] and [6], which show that necessary and sufficient conditions for the von Neumann algebra free product analogous to (54) to be a factor are that if $n_{j}=1$ for some $j$ then for every $1 \leq k \leq K_{2}$

$$
\frac{1}{1-\alpha_{j, 1}} \leq \sum_{i=1}^{m_{k}} \frac{1}{\beta_{k, i}}
$$

and if $m_{j}=1$ for some $j$ then for every $1 \leq k \leq K_{1}$

$$
\frac{1}{1-\beta_{j, 1}} \leq \sum_{i=1}^{n_{k}} \frac{1}{\alpha_{k, i}} .
$$

Moreover, using this von Neumann algebra result and an argument similar to the one used in Proposition 7.2, we see that (55) and (56) are necessary conditions for the simplicity of $\mathfrak{A}$.

For some results about certain reduced free product $\mathrm{C}^{*}$-algebras with respect to non-faithful states, see [10]. 


\section{REFERENCES}

1. J. Anderson, B. Blackadar and U. Haagerup, Minimal projections in the reduced group $C^{*}$ algebra of $\mathbf{Z}_{n} * \mathbf{Z}_{m}$, J. Operator Theory $\mathbf{2 6}$ (1991), 3-23. MR 94c: 46110

2. D. Avitzour, Free products of $C^{*}$-algebras, Trans. Amer. Math. Soc. 271 (1982), 423-465. MR 83h: 46070

3. L.G. Brown, Stable isomorphism of hereditary subalgebras of $C^{*}$-algebras, Pacific J. Math. 71 (1977), 335-348. MR 56:12894

4. L.G. Brown, P. Green, M.A. Rieffel, Stable isomorphism and strong Morita equivalence of $C^{*}$-algebras, Pacific J. Math. 71 (1977), 349-363. MR 57:3866

5. K.J. Dykema, Free products of hyperfinite von Neumann algebras and free dimension, Duke Math. J. 69 (1993), 97-119. MR 93m:46071

6. to non-tracial states, Fields Institute Communications, (D. Voiculescu, editor), vol. 12, 1997, pp. 41-88. MR 98c:46131

7. __ Faithfulness of free product states, J. Funct. Anal. 154(1998), 323-329. CMP 98:11

8. lecture notes, in preparation.

9. K.J. Dykema, U. Haagerup, M. Rørdam, The stable rank of some free product $C^{*}$-algebras, Duke Math. J. 90 (1997), 95-121. CMP 98:03

10. K.J. Dykema, M. Rørdam, Purely infinite simple $C^{*}$-algebras arising from free product constructions, Can. J. Math. 50 (1998), 323-341.

11. E. Germain, KK-theory of reduced free product $C^{*}$-algebras, Duke Math. J. 82 (1996), 707723. MR 97f: 46111

12. E. Germain, $K K$-theory of the full free product of unital $C^{*}$-algebras, J. reine angew. Math. 485 (1997), 1-10. MR 98b:46148

13. R.H. Herman, L.N. Vaserstein, The stable range of $C^{*}$-algebras, Invent. Math. 77 (1984), 553-555. MR 86a:46074

14. W.L. Paschke, N. Salinas, $C^{*}$-algebras associated with free products of groups, Pacific J. Math. 82 (1979), 211-221. MR 82c:22010

15. R.T. Powers, Simplicity of the reduced $C^{*}$-algebra associated with the free group on two generators, Duke Math. J. 42 (1975), 151-156. MR 51:10534

16. M.A. Rieffel, Morita equivalence for operator algebras, Proc. Symp. Pure Math. 38 (1982), 285-298. MR 84k:46045

17. _ Dimension and stable rank in the K-theory of $C^{*}$-algebras, Proc. London Math. Soc. (3) 46 (1983), 301-333. MR 84g:46085

18. M. Rørdam, Advances in the theory of unitary rank and regular approximation, Ann. Math. 128 (1988), 153-172. MR 90c: 46072

19. D. Voiculescu, Symmetries of some reduced free product $C^{*}$-algebras, Operator Algebras and Their Connections with Topology and Ergodic Theory, Lecture Notes in Mathematics, Volume 1132, Springer-Verlag, 1985, pp. 556-588. MR 87d:46075

20. _ Multiplication of certain non-commuting random variables, J. Operator Theory 18 (1987), 223-235. MR 89b:46076

21. D. Voiculescu, K.J. Dykema, A. Nica, Free Random Variables, CRM Monograph Series vol. 1, American Mathematical Society, 1992. MR 94c:46133

Department of Mathematics and Computer Science, Odense University, Campusvej 55, DK-5230 Odense M, Denmark

E-mail address: dykema@imada.ou.dk

URL: http://www.imada.ou.dk/ dykema/ 